\title{
SPECTROSCOPIC SURVEY OF G AND K DWARFS IN THE HIPPARCOS CATALOG. I. COMPARISON BETWEEN THE HIPPARCOS AND PHOTOMETRIC PARALLAXES
}

\author{
Bokyoung Kim ${ }^{1}$, Deokkeun An ${ }^{1,5}$, John R. Stauffer ${ }^{2,5}$, Young Sun LeE ${ }^{3}$, \\ DONAld M. Terndrup ${ }^{4,5}$, and JenNifer A. JOHNSON ${ }^{4}$ \\ ${ }^{1}$ Department of Science Education, Ewha Womans University, 52 Ewhayeodae-gil, Seodaemun-gu, Seoul 03760, Korea; deokkeun@ewha.ac.kr \\ ${ }^{2}$ Spitzer Science Center, California Institute of Technology, Pasadena, CA 91125, USA \\ ${ }^{3}$ Department of Astronomy and Space Science, Chungnam National University, 99 Daehak-ro, Daejeon 34134, Korea \\ ${ }^{4}$ Department of Astronomy, The Ohio State University, 140 West 18th Avenue, Columbus, OH 43210, USA \\ Received 2015 October 8; accepted 2016 January 6; published 2016 February 10
}

\begin{abstract}
The tension between the Hipparcos parallax of the Pleiades and other independent distance estimates continues even after the new reduction of the Hipparcos astrometric data and the development of a new geometric distance measurement for the cluster. A short Pleiades distance from the Hipparcos parallax predicts a number of stars in the solar neighborhood that are sub-luminous at a given photospheric abundance. We test this hypothesis using the spectroscopic abundances for a subset of stars in the Hipparcos catalog, which occupy the same region as the Pleiades in the color-magnitude diagram. We derive stellar parameters for 170 nearby G- and K-type field dwarfs in the Hipparcos catalog based on high-resolution spectra obtained using KPNO $4 \mathrm{~m}$ echelle spectrograph. Our analysis shows that, when the Hipparcos parallaxes are adopted, most of our sample stars follow empirical colormagnitude relations. A small fraction of stars are too faint compared to main-sequence fitting relations by $\Delta M_{V} \gtrsim 0.3 \mathrm{mag}$, but the differences are marginal at a $2 \sigma$ level, partly due to relatively large parallax errors. On the other hand, we find that the photometric distances of stars showing signatures of youth as determined from lithium absorption line strengths and $R_{\mathrm{HK}}^{\prime}$ chromospheric activity indices are consistent with the Hipparcos parallaxes. Our result is contradictory to a suggestion that the Pleiades distance from main-sequence fitting is significantly altered by stellar activity and/or the young age of its stars, and provides an additional supporting evidence for the longdistance scale of the Pleiades.
\end{abstract}

Key words: open clusters and associations: individual (the Pleiades) - solar neighborhood - stars: abundances stars: distances

\section{INTRODUCTION}

The determination of accurate distances to stars is the key to understanding how stars and the Galaxy have formed and evolved. The Hipparcos mission (Perryman et al. 1997) was especially valuable, providing trigonometric parallaxes for $\sim 10^{5}$ stars to a precision of $1-2$ mas. Therefore, it was a big surprise when the Hipparcos distance to the Pleiades open cluster was in disagreement with distances from the mainsequence (MS) fitting at more than a $3 \sigma$ level (Pinsonneault et al. 1998). To reconcile a short Pleiades from Hipparcos, it has been suggested that the metal abundance of the Pleiades is significantly lower than the solar (Percival et al. 2003), thereby decreasing a distance from MS fitting. However, there are a large number of spectroscopic studies on the cluster's metallicity in the literature, which essentially indicates a near-solar metallicity of the cluster (see references in An et al. 2007b). In addition, an enhanced helium abundance of the cluster was suggested (Belikov et al. 1998), but the expected amount of helium has to be enormous $(Y \approx 0.34)$ in this solar metallicity cluster. An argument was also made that distance estimates from theoretical stellar models have been overestimated for young clusters due to yet unknown, age-related physics (van Leeuwen 1999).

The discrepant Hipparcos result for the Pleiades has subsequently led to many efforts to determine the cluster's

\footnotetext{
${ }^{5}$ Visiting astronomer, Kitt Peak National Observatory, National Optical Astronomy Observatory, which is operated by the Association of Universities for Research in Astronomy (AURA) under a cooperative agreement with the National Science Foundation.
}

distance from binaries and independent parallax measurements (e.g., Munari et al. 2004; Pan et al. 2004; Soderblom et al. 2005). These results confirm the long-distance scale from MS fitting, supporting the hypothesis that the Hipparcos result was in error. The most likely explanation is related to the Hipparcos parallaxes themselves. Pinsonneault et al. (1998) showed that a dozen bright stars near the center of the Pleiades all had virtually the same parallax ( $\sim 9$ mas), which is more than 1 mas larger than the mean parallax for other cluster stars. They attributed this to a local zero-point error of individual stellar parallaxes that are correlated over the Hipparcos' 0.9 deg field of view (see also Narayanan \& Gould 1999). By re-reducing part of the Hipparcos data, Makarov $(2002,2003)$ was able to demonstrate that such correlated errors could explain the discrepant Pleiades parallax estimate. Additional effects may result from the way the Hipparcos data were obtained and analyzed (van Leeuwen 2005; van Leeuwen \& Fantino 2005), and it was hoped that a new reduction of the Hipparcos raw data might resolve the issue.

However, the new reduction of the Hipparcos data (van Leeuwen 2007a, 2007b) still leads to a short distance to the Pleiades. In the most recent analysis, van Leeuwen (2009) found $8.32 \pm 0.13$ mas for the average parallax of the Pleiades, or $(m-M)_{0}=5.40 \pm 0.03(120.2 \pm 1.9 \mathrm{pc})$, which is significantly shorter than the weighted average distance $(m-M)_{0}=5.63 \pm 0.02(133.7 \pm 1.2 \mathrm{pc}) \quad$ from independent astrometric and binary solutions (see references in An et al. 2007b). Moreover, Melis et al. (2014) recently used the Very Long Baseline Interferometry (VLBI) to directly measure 
a geometric distance to the Pleiades, and found $(m-M)_{0}=5.67 \pm 0.02(136.2 \pm 1.2 \mathrm{pc})$, which agrees with the long-distance scale.

An alternative, but indirect test of the Pleiades distance can be made by examining nearby field stars that occupy the same region on a color-magnitude diagram (CMD) as those in the Pleiades. The short Hipparcos distance to the Pleiades predicts a number of stars in the solar neighborhood that are subluminous at a given photospheric abundance. Because the absolute magnitudes $\left(M_{V}\right)$ of stars are sensitive to the photospheric abundance, it is possible to distinguish subluminous stars (with the hypothesized Pleiades-like phenomenon) from normal ones, when accurate metallicity measurements are available. Here, our core assumption is that parallaxes for the majority of stars in the Hipparcos catalog are correct, but only a small fraction of these stars (such as those in the Pleiades) have incorrect parallaxes due to large, hidden systematic errors.

Previously, Soderblom et al. (1998) performed such test using a set of nearby field stars, but found no stars of similar characteristics with the Pleiades members. However, the interpretation of their result is somewhat complicated by the fact that the stars in their sample mostly have spectral types later than K2. Late-type, young, chromospherically active stars can be heavily spotted and hence variable, and their optical colors can differ significantly from those of older field stars of the same spectral type (Stauffer et al. 2003). On the other hand, rapid rotation does not cause significant photometric anomalies of stars with spectral types earlier than $\mathrm{K} 2$.

The goal of this paper is to obtain accurate metal abundances for a subset of G- and early K-type stars in the Hipparcos catalog, and look for sub-luminous field stars at a given metallicity. Such stars will have longer distances from MS fitting than those computed from the Hipparcos parallaxes. Furthermore, if the assertion by van Leeuwen (2009) is correct, and the young age of the Pleiades is responsible for the long MS-fitting distance to the cluster, young field stars, such as those selected from strong lithium absorptions or $\mathrm{Ca}$ II $\mathrm{H}$ and $\mathrm{K}$ emissions, will be fainter than their older counterparts by $\Delta M_{V} \gtrsim 0.2 \mathrm{mag}$ at a common $[\mathrm{Fe} / \mathrm{H}]$. Assuming a constant star formation rate and an age of $\sim 8 \mathrm{Gyr}$ for the thin disk stellar population (e.g., see Casagrande et al. 2016, and references therein), about $2.5 \%$ of stars in the solar neighborhood were formed in the last $200 \mathrm{Myr}$. A couple of young stars should be present in a sample of $\sim 100$ field stars. The expected number of such stars will decrease if an exponentially decreasing star formation rate is assumed (e.g., Aumer \& Binney 2009), but a relatively large number of young open clusters in the solar neighborhood implies the presence of many young stars near the Sun. Furthermore, a vertical age gradient in the disk (e.g., Casagrande et al. 2016) would yield more young stars near the Galactic plane. A well-defined set of field stars can be used to disprove a null hypothesis that the short distance to the Pleiades is caused by the young age of the cluster.

This paper is organized as follows. In Section 2 we describe spectroscopic observations and data reductions. Derivation of stellar parameter is given in Section 3. In Section 4 we derive MS-fitting distances for individual stars using spectroscopic metallicities, and compare them to the Hipparcos parallaxes. A summary of our results is given in Section 5.

\section{SPECTROSCOPIC OBSERVATIONS AND DATA REDUCTIONS}

\subsection{Sample Selection}

The top lefthand panel in Figure 1 illustrates our sample selection based on a $B-V$ CMD. Gray points are stars with good parallaxes $\left(\sigma_{\pi} / \pi \leqslant 0.07\right)$ in the revised Hipparcos catalog (van Leeuwen 2007a, 2007b). We took $B V$ photometry of these stars from the NASA Star and Exoplanet Database (NStED), most of which are those transformed from $B_{T} V_{T}$ in the Tycho-2 catalog (Høg et al. 2000) using transformation equations found in Mamajek et al. (2002, 2006). We neglected foreground extinctions of these stars because they are mostly found within $\sim 50 \mathrm{pc}$ from the Sun.

The parallelogram in the top lefthand panel of Figure 1 indicates our color-magnitude selection, corresponding to $4.74 \leqslant M_{V} \leqslant 6.34$ at $B-V=0.60$ and $6.86 \leqslant M_{V} \leqslant 8.46$ at $B-V=1.0$. The bluer color limit was set to minimize the evolutionary effect on stellar luminosity and to perform a reliable line absorption analysis. We selected stars near or below the MS of the Pleiades on the absolute $V$ magnitude $\left(M_{V}\right)$ versus $B-V \mathrm{CMD}$, assuming a distance of the cluster from the recent Hipparcos parallax, $(m-M)_{0}=5.40$ (van Leeuwen 2009). We retained stars if they are found within $\Delta M_{V} \sim 1.4$ mag below and $\Delta M_{V} \sim 0.2 \mathrm{mag}$ above the MS of the Pleiades, as shown by the top and the bottom sides of the parallelogram. We applied a color limit $B-V=1.0$ to exclude chromospherically active low-mass stars with large color anomalies (Stauffer et al. 2003).

In the top lefthand panel of Figure 1, the thick solid line represents the observed MS of the Hyades (Pinsonneault et al. 2004). We adopted a distance to the cluster's center of mass $(m-M)_{0}=3.33 \pm 0.01$ from the Hipparcos catalog (Perryman et al. 1998). The Hyades covers a large area on the sky, which makes the parallax measurements of its individual members less vulnerable to the suspected spatial correlation of the Hipparcos parallax (Pinsonneault et al. 1998; Narayanan \& Gould 1999; de Bruijne et al. 2001). The cluster is approximately $550 \mathrm{Myr}$ old (Perryman et al. 1998) and has $[\mathrm{Fe} / \mathrm{H}]=+0.13 \pm 0.01$ (Paulson et al. 2003) with negligible foreground reddening (e.g., Taylor 1980). The two thin solid lines are 550 Myr old theoretical models at $[\mathrm{Fe} / \mathrm{H}]=-0.3$ and $[\mathrm{Fe} / \mathrm{H}]=0.0$ (An et al. 2007b), of which colors were calibrated using the observed MS of the Hyades. All together, these lines show a typical metallicity sensitivity of colors and magnitudes of MS stars. In this study, however, we avoided using theoretical isochrones and relied on the observed MS of the Hyades to empirically derive a MS-fitting distance to individual field stars.

Meanwhile, the top of the parallelogram in Figure 1 is not exactly parallel to the solar metallicity isochrone. This is because the Pleiades' MS, which was used to set our sample color-magnitude cut, is known to become progressively fainter than those for older stars or standard stellar models as one moves toward redder colors (Stauffer et al. 2003; An et al. 2007b), although the observed magnitude offset in the $T_{\text {eff }}$ range of our sample is not as severe as those seen for stars with $B-V \gtrsim 1$ (see Figure 20 in An et al. 2007b). Nevertheless, our search for anomalously faint stars in the solar neighborhood is almost insensitive to how we set the upper limit in stellar brightness, because such stars would be 

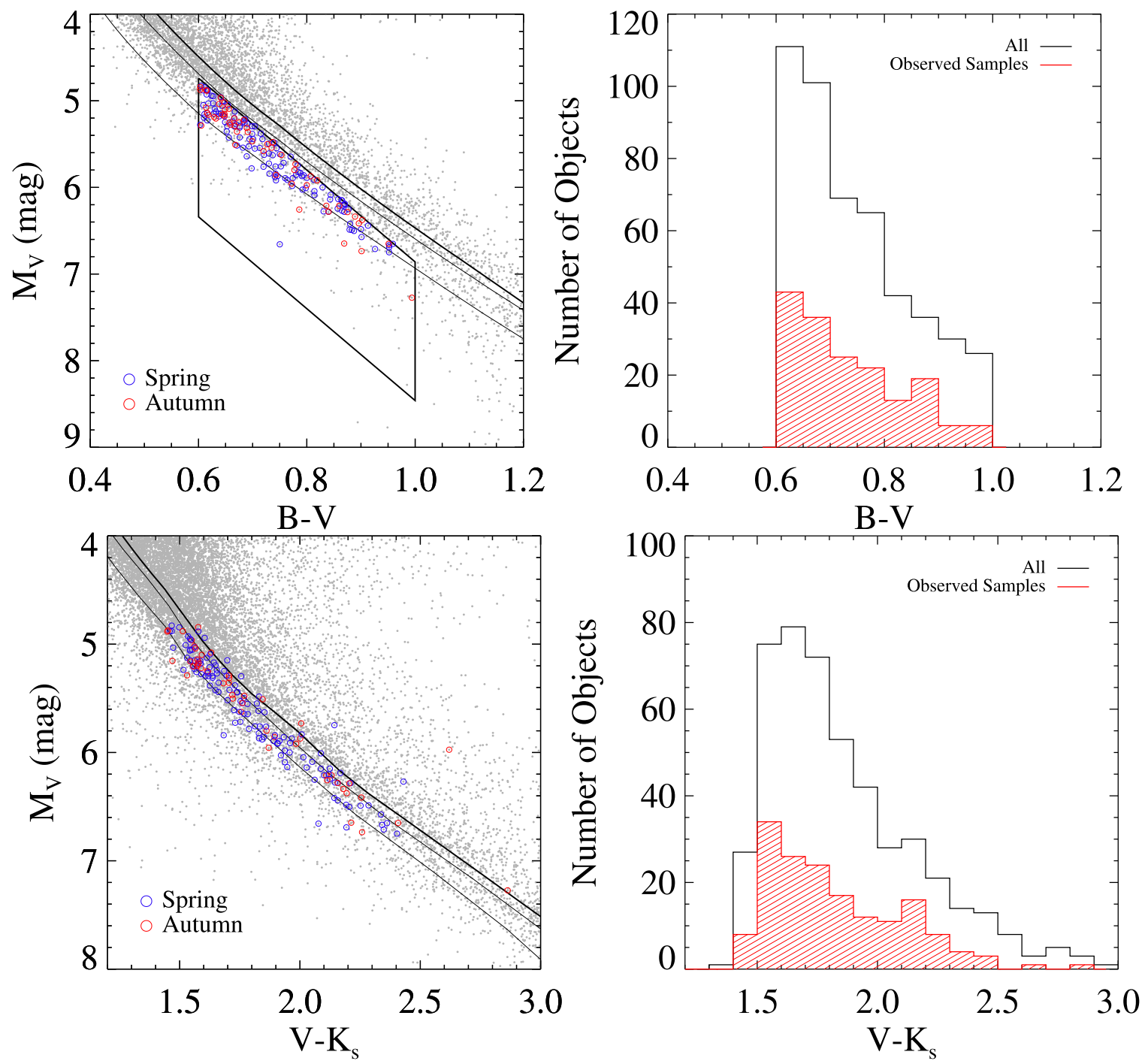

Figure 1. Top left: color-magnitude diagram of field stars in the solar neighborhood with Hipparcos parallaxes $\left(\sigma_{\pi} / \pi \leqslant 0.07\right)$. The parallelogram represents a colormagnitude selection of spectroscopic targets in this study, where the KPNO samples observed in the spring $(N=120)$ and autumn $(N=53)$ runs are shown in blue and red circles, respectively. The thick solid line is the observed MS of the Hyades $([\mathrm{Fe} / \mathrm{H}]=+0.13)$ at the Hipparcos distance to the cluster (Perryman et al. 1998), while thin lines are theoretical isochrones with empirical colors at $[\mathrm{Fe} / \mathrm{H}]=-0.3$ and 0.0 , respectively (An et al. 2015). Bottom left: same as in the top lefthand panel, but with $V-K_{s}$ colors. Right panels: color distributions of the KPNO sample stars (red histogram) in $B-V$ (top) and $V-K_{s}$ (bottom), respectively. The black histogram represents a distribution of all stars in the Hipparcos catalog $\left(\sigma_{\pi} / \pi \leqslant 0.07\right)$ that are found within the parallelogram in the top lefthand panel.

significantly fainter than the above brightness limit (see discussions in Section 4.2).

In total $480 \mathrm{G}$ - and K-type dwarfs satisfy this selection criteria. We combined the $V$-band magnitude with $K_{s}$ in the Two Micron All Sky Survey (2MASS; Skrutskie et al. 2006). ${ }^{6}$ With the exception of five stars (HIP 9172, HIP 56837, HIP 91438, HIP 96100, and HIP 101997), our targets have valid $K_{s}$-band measurements, which are not saturated, undetected, blended, or contaminated. The bottom lefthand panel in Figure 1 shows the same set of stars in the $V-K_{s} \mathrm{CMD}$, although we did not employ $V-K_{s}$ colors in our sample selection.

\footnotetext{
6 This publication makes use of data products from the Two Micron All Sky Survey, which is a joint project of the University of Massachusetts and the Infrared Processing and Analysis Center/California Institute of Technology, funded by the National Aeronautics and Space Administration and the National Science Foundation.
}

\subsection{Observations and Data Reductions}

We selected a random subset of Hipparcos field stars that satisfy our color-magnitude cut, and obtained their highresolution $(\lambda / \Delta \lambda \sim 60,000)$ spectra with the echelle spectrograph on the Mayall $4 \mathrm{~m}$ telescope at Kitt Peak National Observatory (KPNO). Most of our targets are bright $(V \sim 9 \mathrm{mag}$ ) and spread all around sky, except those with low declinations $\left(\delta \lesssim-30^{\circ}\right)$ due to observing restrictions at KPNO. Our observing campaign was composed of five nights in 2010 May and four nights in 2010 September. In the lefthand panels of Figure 1, targets observed in spring and autumn are marked in blue and red circles, respectively. Unresolved binaries are cooler than single stars and therefore better represented in $V-K_{s}$ thanks to its longer wavelength baseline. Our color-magnitude cut is likely smeared out in the $V-K_{S}$ CMD by these unresolved binaries, along with photometric color errors. The $B-V$ and $V-K_{s}$ color distributions of our observed sample are shown by a red shaded histogram in the righthand panels in Figure 1. 
The open histogram represents a distribution of the initial sample selected above from the Hipparcos catalog (those within a parallelogram in the top lefthand panel). The overall shapes of the red shaded and open histograms in each panel are similar to one another, as expected from a random selection of stars in our spectroscopic observations.

The sky was clear in spring, but the dome was closed during two nights in autumn due to bad weather conditions. We used a red long camera and settled on the 58.5-63 grating with a $\sim 1^{\prime \prime}$ slit width. With the $2048 \times 2048$ T2KB CCD and 226-1 cross disperser, the wavelength coverages were set to $4340 \AA-$ $7670 \AA$ in May, and $4400 \AA-7870 \AA$ in September. The spectra are of high quality, with high signal-to-noise ratios $(\mathrm{S} / \mathrm{N})(>100$ per pixel).

In total, 120 stars were observed in spring and 53 stars were observed in autumn. Three stars were observed in both the spring and autumn runs (HIP 113884, HIP 104733, HIP 98792). From our observing runs, spectra for 170 field stars in the Hipparcos catalog were obtained. Table 1 lists our sample stars with $V, B-V$, and $V-K_{s}$ colors and their errors from the Hipparcos catalog. A minimum error in $V$ and $B-V$ was set to $0.02 \mathrm{mag}$. The 9 th and 10 th columns show revised Hipparcos parallaxes $(\pi)$ and their errors (van Leeuwen 2007a, 2007b). The $M_{V}$ and its error in the next two columns were computed using $V$ and $\pi$ and their associated errors. Seven targets were identified as a spectroscopic binary system in the 9th catalog of spectroscopic binary orbits (Pourbaix et al. 2004), ${ }^{7}$ and are marked in Table 1. The number of repeat observations for each target is indicated in the following columns. About a third of the sample stars $(N=69)$ were observed two to three times, and eight stars were observed on two different nights to check for systematics in our abundance measurements.

We reduced raw data frames using standard data processing packages in IRAF. ${ }^{8}$ This included a bias correction, bad pixel correction, wavelength calibration using Th-Ar lamp, spectral extraction, and radial velocity correction. For data taken in autumn, we found a uniformly increasing bias pattern toward one side of the CCD, which consistently appeared over the entire observing run. We combined all of the zero-exposure frames, made an average bias frame, and subtracted it from our science data frames. We corrected for a wavelength shift from a line-of-sight velocity using the $\mathrm{H} \alpha$ line profile. When the $\mathrm{H} \alpha$ line was not available, the $\mathrm{Na}$ I 5890.0 A line was used for a radial velocity correction.

\section{DERIVATION OF STELLAR PARAMETERS}

Our conclusions about stars with the Hipparcos parallaxes are sensitive to the adopted sizes of errors in metallicity, while stellar parameters-effective temperatures $\left(T_{\text {eff }}\right)$, surface gravity $(\log g)$, and metallicity $([\mathrm{Fe} / \mathrm{H}])$ - derived from spectroscopy are subject to various systematic errors. Because such errors could originate from different spectroscopic analysis techniques (e.g., Torres et al. 2012), we derived stellar parameters in two parallel approaches. We employed a spectral synthesis code MOOG $^{9}$ (Sneden 1973) based on equivalent

\footnotetext{
7 http://sb9.astro.ulb.ac.be

8 IRAF is distributed by National Optical Astronomy Observatories (NOAO), which is operated by the Association of Universities for Research in Astronomy, Inc., under arrangement with the National Science Foundation, United States.

9 http://www.as.utexas.edu/ chris/moog.html
}

width (EW) measurements of iron lines (Section 3.1). Because this procedure was performed by hand, we restricted our MOOG analysis to a subset of stars in the sample $(N=74$ out of 170) with highly precise parallax measurements $\left(\sigma_{\pi} / \pi \leqslant 0.03\right)$, and those $(N=13)$ having largest $M_{V}$ differences between Hipparcos and MS fitting despite their less accurate parallaxes $\left(\sigma_{\pi} / \pi>0.03\right)$. For the entire sample (including those analyzed using MOOG), we employed an automated spectral matching technique (SMT) that iteratively fits synthetic spectra to an observed spectrum to search for the best matching parameters with the least human intervention. For an efficient estimation of stellar parameters with SMT, we degraded our spectra to a medium resolution $(R=10,000)$, while keeping the same precision in the stellar parameter estimates. Below we describe each of these approaches, along with external checks with previous work in the literature.

\subsection{Stellar Parameters from $M O O G$}

We selected stars with accurate parallaxes $\left(\sigma_{\pi} / \pi \leqslant 0.03\right)$ in our sample $(N=74)$, and used MOOG to derive their precise atmospheric parameters, taking advantage of high-resolution $(R \sim 60,000)$ spectra of high quality $(\mathrm{S} / \mathrm{N}>100)$. We selected a list of Fe lines that were commonly included in both Bensby et al. (2003) and Boesgaard et al. (2013). Within the wavelength range of our echelle data, there were $37 \mathrm{Fe}$ I lines and $5 \mathrm{Fe}$ II lines. These lines are listed in Table 2 along with their central wavelengths, excitation potentials (EP), and oscillator strengths $(\log g f)$. We determined a local continuum for each absorption line using a polynomial with a degree of 3 , and measured its EW using SPECTRE ${ }^{10}$ (Sneden, version 2003).

We constructed Kurucz stellar model atmospheres based on newly computed opacity distribution functions (ODFs) with updated opacities and abundances (Castelli \& Kurucz 2004). ${ }^{11}$ We interpolated ODFs at a given set of $[\mathrm{Fe} / \mathrm{H}]$ and microturbulence $\xi$, and constructed desired models using the ATLAS9 code of stellar atmosphere. We assumed solar abundance ratios in Grevesse \& Sauval (1999) with an enhancement in the $\alpha$-elements ratios $(\mathrm{O}, \mathrm{Mg}, \mathrm{Si}, \mathrm{Ca}$, and $\mathrm{Ti})$ relative to $\mathrm{Fe}$ : +0.4 for $[\mathrm{Fe} / \mathrm{H}] \leqslant-1.0,+0.3$ for $[\mathrm{Fe} / \mathrm{H}]=-0.75, \quad+0.2$ for $[\mathrm{Fe} / \mathrm{H}]=-0.5, \quad+0.1$ for $[\mathrm{Fe} / \mathrm{H}]=-0.25$, and 0.0 for $[\mathrm{Fe} / \mathrm{H}] \geqslant+0.0$.

We used the abfind driver in MOOG to self-consistently constrain $T_{\text {eff }}, \log g,[\mathrm{Fe} / \mathrm{H}]$, and microturbulence $(\xi)$. The effective temperature was derived by requiring that individual line abundances be independent of excitation potential and $\xi$ be independent of line strength. Insisting on ionization equilibrium between $\mathrm{Fe}_{\mathrm{I}}$ and $\mathrm{Fe}$ II allowed for a simultaneous determination of $\log g$ with $T_{\text {eff }}$ and $\xi$. Our sample stars are relatively cool, and therefore non-LTE corrections are likely negligible (see Bensby et al. 2014).

Table 3 lists $T_{\text {eff }}, \log g,[\mathrm{Fe} / \mathrm{H}]$, and $\xi$ of the stars analyzed using MOOG. In addition to 74 stars with accurate parallax measurements, we included 13 stars in our MOOG analysis that show large differences in distance between Hipparcos and MS fitting (see Section 4.2). We adopted a solar Fe abundance $A(\mathrm{Fe})^{12}=7.52$ in Anders \& Grevesse $(1989)$. The $[\mathrm{Fe} / \mathrm{H}]_{\text {corr }}$

\footnotetext{
${ }^{10}$ http://www.as.utexas.edu/ chris/spectre.html

11 http://kurucz.harvard.edu/grids.html

$12 A(\mathrm{Fe}) \equiv \log [N(\mathrm{Fe}) / N(\mathrm{H})]+12$.
} 
Table 1

Photometric Properties of the KPNO Sample

\begin{tabular}{|c|c|c|c|c|c|c|c|c|c|c|c|c|c|}
\hline \multirow[t]{2}{*}{ HIP } & \multirow[t]{2}{*}{ HD } & \multirow{2}{*}{$\begin{array}{c}V \\
(\mathrm{mag})\end{array}$} & \multirow{2}{*}{$\begin{array}{l}\sigma(V) \\
(\mathrm{mag})\end{array}$} & \multirow{2}{*}{$\begin{array}{l}B-V \\
(\mathrm{mag})\end{array}$} & \multirow{2}{*}{$\begin{array}{l}\sigma_{B}-V \\
(\mathrm{mag})\end{array}$} & \multirow{2}{*}{$\begin{array}{l}V-K_{s} \\
(\mathrm{mag})\end{array}$} & \multirow{2}{*}{$\begin{array}{l}\sigma_{V-K_{S}} \\
(\mathrm{mag})\end{array}$} & \multirow{2}{*}{$\begin{array}{c}\pi^{\mathrm{a}} \\
(\operatorname{arcsec})\end{array}$} & \multirow{2}{*}{$\begin{array}{c}\sigma_{\pi}^{\mathrm{a}} \\
(\operatorname{arcsec})\end{array}$} & $M_{V}^{\mathrm{b}}$ & $\sigma\left(M_{V}\right)$ & Numb & of Obs. \\
\hline & & & & & & & & & & (mag) & (mag) & Spring & Autumn \\
\hline 1674 & 1624 & 8.48 & 0.02 & 0.80 & 0.02 & 2.62 & 0.03 & 31.54 & 0.96 & 5.97 & 0.11 & $\ldots$ & 2 \\
\hline 4907 & 5996 & 7.66 & 0.02 & 0.75 & 0.02 & 1.76 & 0.03 & 39.20 & 0.56 & 5.63 & 0.10 & $\ldots$ & 1 \\
\hline 5313 & 6664 & 7.78 & 0.02 & 0.61 & 0.02 & 1.53 & 0.03 & 31.79 & 1.66 & 5.29 & 0.13 & $\ldots$ & 2 \\
\hline 5521 & 6963 & 7.66 & 0.02 & 0.73 & 0.02 & 1.72 & 0.03 & 36.95 & 0.79 & 5.50 & 0.10 & $\ldots$ & 1 \\
\hline 9172 & 11926 & 7.57 & 0.02 & 0.66 & 0.02 & $\ldots$ & & 31.99 & 0.86 & 5.09 & 0.06 & $\ldots$ & 1 \\
\hline 9829 & 12846 & 6.89 & 0.02 & 0.65 & 0.02 & 1.59 & 0.03 & 43.89 & 0.57 & 5.10 & 0.04 & $\ldots$ & 2 \\
\hline 10276 & 13483 & 8.46 & 0.02 & 0.77 & 0.03 & 1.87 & 0.03 & 31.58 & 1.11 & 5.96 & 0.11 & $\ldots$ & 2 \\
\hline 10629 & 13783 & 8.29 & 0.02 & 0.69 & 0.02 & 1.71 & 0.03 & 25.37 & 0.93 & 5.31 & 0.08 & $\ldots$ & 2 \\
\hline 11507 & 15361 & 8.60 & 0.02 & 0.66 & 0.03 & 1.62 & 0.03 & 21.44 & 0.89 & 5.25 & 0.04 & $\ldots$ & 2 \\
\hline 12685 & 16702 & 8.29 & 0.02 & 0.60 & 0.02 & 1.58 & 0.03 & 20.44 & 0.93 & 4.84 & 0.11 & $\ldots$ & 1 \\
\hline 13771 & 18123 & 8.80 & 0.02 & 0.67 & 0.03 & 1.71 & 0.03 & 19.87 & 1.15 & 5.29 & 0.10 & $\ldots$ & 2 \\
\hline 14241 & 19034 & 8.08 & 0.02 & 0.67 & 0.03 & 1.70 & 0.03 & 28.52 & 0.97 & 5.36 & 0.04 & $\ldots$ & 2 \\
\hline 14300 & 18916 & 7.99 & 0.02 & 0.68 & 0.02 & 1.59 & 0.03 & 27.97 & 0.94 & 5.22 & 0.07 & $\ldots$ & 2 \\
\hline 15062 & 20065 & 8.13 & 0.02 & 0.62 & 0.02 & 1.58 & 0.03 & 25.52 & 0.90 & 5.16 & 0.14 & $\ldots$ & 1 \\
\hline 15442 & 20619 & 7.03 & 0.02 & 0.65 & 0.02 & 1.56 & 0.03 & 39.64 & 0.74 & 5.02 & 0.15 & $\ldots$ & 1 \\
\hline 17265 & 23065 & 8.27 & 0.02 & 0.74 & 0.03 & 1.89 & 0.04 & 32.89 & 0.74 & 5.86 & 0.09 & $\ldots$ & 1 \\
\hline 18324 & 24238 & 7.82 & 0.02 & 0.86 & 0.02 & 2.13 & 0.03 & 47.60 & 0.84 & 6.21 & 0.10 & $\ldots$ & 1 \\
\hline 20722 & 27857 & 8.05 & 0.02 & 0.64 & 0.02 & 1.55 & 0.03 & 24.12 & 0.87 & 4.96 & 0.11 & $\ldots$ & 1 \\
\hline 21276 & 28495 & 7.76 & 0.02 & 0.78 & 0.02 & 2.01 & 0.03 & 39.37 & 1.11 & 5.73 & 0.11 & $\ldots$ & 1 \\
\hline 21571 & 29021 & 7.76 & 0.02 & 0.69 & 0.02 & 1.68 & 0.03 & 33.07 & 0.77 & 5.36 & 0.04 & $\ldots$ & 2 \\
\hline $21832^{c}$ & 29587 & 7.28 & 0.02 & 0.61 & 0.02 & 1.63 & 0.03 & 36.30 & 0.87 & 5.08 & 0.11 & $\ldots$ & 1 \\
\hline 22175 & 30286 & 7.83 & 0.02 & 0.68 & 0.02 & 1.61 & 0.03 & 30.53 & 0.82 & 5.25 & 0.02 & $\ldots$ & 1 \\
\hline 23431 & 32237 & 8.19 & 0.02 & 0.78 & 0.02 & 1.86 & 0.03 & 33.33 & 1.42 & 5.80 & 0.03 & $\ldots$ & 1 \\
\hline 23786 & 32850 & 7.74 & 0.02 & 0.81 & 0.02 & 2.00 & 0.03 & 42.25 & 0.92 & 5.87 & 0.04 & $\ldots$ & 1 \\
\hline 26505 & 37008 & 7.74 & 0.02 & 0.84 & 0.02 & 2.11 & 0.03 & 49.59 & 0.72 & 6.21 & 0.12 & $\ldots$ & 2 \\
\hline 30862 & 45391 & 7.14 & 0.02 & 0.63 & 0.02 & 1.57 & 0.03 & 40.70 & 0.62 & 5.19 & 0.09 & $\ldots$ & 2 \\
\hline $44259^{c}$ & 77065 & 8.78 & 0.02 & 0.83 & 0.04 & 2.15 & 0.03 & 31.54 & 1.05 & 6.28 & 0.05 & 2 & $\ldots$ \\
\hline 44262 & 77052 & 8.84 & 0.02 & 0.64 & 0.04 & 1.55 & 0.03 & 16.75 & 1.11 & 4.96 & 0.04 & 2 & $\ldots$ \\
\hline 52278 & 92320 & 8.38 & 0.02 & 0.69 & 0.02 & 1.57 & 0.03 & 23.79 & 0.78 & 5.26 & 0.07 & 1 & $\ldots$ \\
\hline 52470 & 92786 & 7.98 & 0.02 & 0.78 & 0.02 & 1.85 & 0.03 & 37.54 & 0.76 & 5.86 & 0.10 & 1 & $\ldots$ \\
\hline 54906 & 97658 & 7.76 & 0.02 & 0.84 & 0.02 & 2.03 & 0.03 & 47.36 & 0.75 & 6.14 & 0.14 & 1 & $\ldots$ \\
\hline 55210 & 98281 & 7.29 & 0.02 & 0.75 & 0.02 & 1.83 & 0.04 & 46.37 & 0.64 & 5.62 & 0.12 & 2 & $\ldots$ \\
\hline 56092 & 238006 & 9.69 & 0.02 & 0.95 & 0.04 & 2.19 & 0.03 & 25.17 & 1.55 & 6.69 & 0.02 & 2 & $\ldots$ \\
\hline 56337 & 100310 & 8.82 & 0.02 & 0.73 & 0.03 & 1.73 & 0.03 & 22.79 & 1.16 & 5.61 & 0.05 & 2 & $\ldots$ \\
\hline 56837 & 101227 & 8.44 & 0.02 & 0.68 & 0.02 & $\ldots$ & $\ldots$ & 26.34 & 0.88 & 5.54 & 0.14 & 1 & $\ldots$ \\
\hline 57939 & 103095 & 6.45 & 0.02 & 0.75 & 0.02 & 2.08 & 0.03 & 109.98 & 0.41 & 6.66 & 0.12 & 2 & $\ldots$ \\
\hline 57992 & 103126 & 8.29 & 0.02 & 0.74 & 0.02 & 1.84 & 0.03 & 32.98 & 0.56 & 5.88 & 0.11 & 1 & $\ldots$ \\
\hline 58949 & 104988 & 8.17 & 0.02 & 0.74 & 0.02 & 1.85 & 0.03 & 30.97 & 0.85 & 5.63 & 0.12 & 1 & $\ldots$ \\
\hline 60074 & 107146 & 7.04 & 0.02 & 0.61 & 0.02 & 1.50 & 0.03 & 36.42 & 0.53 & 4.84 & 0.06 & 2 & $\ldots$ \\
\hline $60268^{c}$ & 107582 & 8.25 & 0.02 & 0.61 & 0.02 & 1.65 & 0.03 & 24.48 & 0.62 & 5.20 & 0.03 & 1 & $\ldots$ \\
\hline 63322 & 112733B & 9.31 & 0.02 & 0.87 & 0.04 & 2.43 & 0.03 & 24.64 & 1.67 & 6.27 & 0.07 & 2 & $\ldots$ \\
\hline 63346 & 112956 & 8.06 & 0.02 & 0.69 & 0.02 & 1.74 & 0.03 & 30.00 & 0.56 & 5.44 & 0.08 & 1 & $\ldots$ \\
\hline 63636 & 113319 & 7.51 & 0.02 & 0.65 & 0.02 & 1.54 & 0.03 & 32.15 & 0.56 & 5.05 & 0.07 & 2 & $\ldots$ \\
\hline 64076 & 114216 & 8.50 & 0.02 & 0.61 & 0.02 & 1.55 & 0.03 & 20.40 & 0.62 & 5.05 & 0.07 & 2 & $\ldots$ \\
\hline 64131 & 114172 & 8.56 & 0.02 & 0.62 & 0.02 & 1.56 & 0.03 & 21.56 & 0.88 & 5.23 & 0.13 & 2 & $\ldots$ \\
\hline 65165 & 116272 & 8.48 & 0.02 & 0.77 & 0.02 & 1.78 & 0.03 & 27.05 & 0.63 & 5.64 & 0.08 & 1 & $\ldots$ \\
\hline 65352 & 116442 & 7.03 & 0.02 & 0.81 & 0.02 & 1.94 & 0.03 & 64.73 & 1.33 & 6.09 & 0.13 & 2 & $\ldots$ \\
\hline 65355 & 116443 & 7.29 & 0.02 & 0.88 & 0.02 & 2.06 & 0.03 & 61.94 & 1.34 & 6.25 & 0.12 & 1 & $\ldots$ \\
\hline 65935 & $\ldots$ & 9.63 & 0.02 & 0.90 & 0.04 & 2.19 & 0.03 & 23.45 & 1.35 & 6.48 & 0.02 & 2 & $\ldots$ \\
\hline 66509 & 118659 & 8.83 & 0.02 & 0.65 & 0.03 & 1.70 & 0.03 & 19.64 & 1.13 & 5.29 & 0.15 & 1 & $\ldots$ \\
\hline 67211 & 119932 & 9.31 & 0.03 & 0.95 & 0.05 & 2.40 & 0.03 & 30.75 & 1.42 & 6.75 & 0.12 & 2 & $\ldots$ \\
\hline 67282 & 120067 & 8.70 & 0.02 & 0.83 & 0.03 & 1.96 & 0.03 & 28.91 & 0.97 & 6.00 & 0.12 & 2 & $\ldots$ \\
\hline 67773 & 121131 & 8.36 & 0.02 & 0.81 & 0.02 & 2.01 & 0.03 & 34.41 & 1.22 & 6.04 & 0.04 & 1 & $\ldots$ \\
\hline 67904 & 121320 & 7.88 & 0.02 & 0.70 & 0.02 & 1.63 & 0.03 & 31.73 & 0.71 & 5.38 & 0.12 & 3 & $\ldots$ \\
\hline 70253 & 126244 & 8.23 & 0.02 & 0.61 & 0.02 & 1.59 & 0.03 & 24.27 & 0.64 & 5.16 & 0.13 & 1 & $\ldots$ \\
\hline 70319 & 126053 & 6.27 & 0.02 & 0.63 & 0.02 & 1.63 & 0.03 & 58.17 & 0.52 & 5.09 & 0.05 & 3 & $\ldots$ \\
\hline 71720 & 128985 & 9.17 & 0.02 & 0.86 & 0.04 & 1.95 & 0.03 & 24.74 & 1.37 & 6.14 & 0.06 & 1 & $\ldots$ \\
\hline 72577 & 130871 & 9.06 & 0.02 & 0.95 & 0.04 & 2.34 & 0.03 & 33.18 & 1.85 & 6.67 & 0.13 & 2 & $\ldots$ \\
\hline 72663 & 131025 & 8.48 & 0.02 & 0.68 & 0.03 & 1.64 & 0.03 & 23.25 & 1.30 & 5.31 & 0.10 & 1 & $\ldots$ \\
\hline 72703 & 131179 & 8.36 & 0.02 & 0.70 & 0.03 & 1.66 & 0.03 & 26.08 & 0.87 & 5.44 & 0.03 & 1 & $\ldots$ \\
\hline 73005 & 132142 & 7.76 & 0.02 & 0.81 & 0.02 & 1.96 & 0.03 & 42.75 & 0.45 & 5.91 & 0.08 & 1 & $\ldots$ \\
\hline 73138 & 132051 & 8.65 & 0.02 & 0.77 & 0.03 & 1.94 & 0.03 & 29.25 & 1.60 & 5.98 & 0.08 & 1 & $\ldots$ \\
\hline 73677 & 133564 & 8.80 & 0.02 & 0.63 & 0.02 & 1.54 & 0.03 & 18.58 & 0.74 & 5.15 & 0.10 & 1 & $\ldots$ \\
\hline
\end{tabular}


Table 1

(Continued)

\begin{tabular}{|c|c|c|c|c|c|c|c|c|c|c|c|c|c|}
\hline \multirow[t]{2}{*}{ HIP } & \multirow[t]{2}{*}{ HD } & \multirow{2}{*}{$\begin{array}{c}V \\
(\mathrm{mag})\end{array}$} & \multirow{2}{*}{$\begin{array}{l}\sigma(V) \\
(\mathrm{mag})\end{array}$} & \multirow{2}{*}{$\begin{array}{l}B-V \\
(\mathrm{mag})\end{array}$} & \multirow{2}{*}{$\begin{array}{l}\sigma_{B-V} \\
(\mathrm{mag})\end{array}$} & \multirow{2}{*}{$\begin{array}{l}V-K_{s} \\
(\mathrm{mag})\end{array}$} & \multirow{2}{*}{$\begin{array}{l}\sigma_{V-K_{S}} \\
(\mathrm{mag})\end{array}$} & \multirow{2}{*}{$\begin{array}{c}\pi^{\mathrm{a}} \\
(\operatorname{arcsec})\end{array}$} & \multirow{2}{*}{$\begin{array}{c}\sigma_{\pi}^{\mathrm{a}} \\
(\operatorname{arcsec})\end{array}$} & $M_{V}^{\mathrm{b}}$ & $\sigma\left(M_{V}\right)$ & Numb & of Obs. \\
\hline & & & & & & & & & & (mag) & (mag) & Spring & Autumn \\
\hline 73845 & 133910 & 9.10 & 0.02 & 0.63 & 0.03 & 1.56 & 0.03 & 15.36 & 0.90 & 5.03 & 0.09 & 1 & $\ldots$ \\
\hline 74126 & 134251 & 8.88 & 0.02 & 0.66 & 0.03 & 1.65 & 0.04 & 20.33 & 1.09 & 5.42 & 0.13 & 1 & $\ldots$ \\
\hline 74396 & 135144 & 8.59 & 0.02 & 0.87 & 0.02 & 2.12 & 0.03 & 33.27 & 0.76 & 6.20 & 0.10 & 1 & $\ldots$ \\
\hline 75059 & 136563 & 8.57 & 0.02 & 0.67 & 0.02 & 1.53 & 0.03 & 20.50 & 1.04 & 5.12 & 0.06 & 1 & $\ldots$ \\
\hline 75370 & 137826 & 8.74 & 0.02 & 0.67 & 0.03 & 1.76 & 0.03 & 20.43 & 0.73 & 5.30 & 0.09 & 1 & $\ldots$ \\
\hline 75446 & 137336 & 8.98 & 0.02 & 0.73 & 0.03 & 1.73 & 0.03 & 22.37 & 1.15 & 5.73 & 0.14 & 1 & $\ldots$ \\
\hline 75703 & 138134 & 9.05 & 0.02 & 0.75 & 0.03 & 1.83 & 0.03 & 23.14 & 0.79 & 5.87 & 0.13 & 1 & $\ldots$ \\
\hline 76058 & 138442 & 8.73 & 0.02 & 0.64 & 0.03 & 1.59 & 0.03 & 19.55 & 1.10 & 5.18 & 0.08 & 2 & $\ldots$ \\
\hline 76130 & $\ldots$ & 8.98 & 0.02 & 0.61 & 0.03 & 1.47 & 0.03 & 15.15 & 0.94 & 4.88 & 0.08 & 1 & $\ldots$ \\
\hline 76330 & 139194 & 8.62 & 0.02 & 0.86 & 0.02 & 2.12 & 0.04 & 33.50 & 0.83 & 6.25 & 0.11 & 1 & $\ldots$ \\
\hline 76674 & 139837 & 9.00 & 0.02 & 0.74 & 0.03 & 1.82 & 0.03 & 20.37 & 1.05 & 5.55 & 0.12 & 1 & $\ldots$ \\
\hline 77810 & 142229 & 8.08 & 0.02 & 0.62 & 0.02 & 1.47 & 0.03 & 24.53 & 1.25 & 5.03 & 0.15 & 1 & $\ldots$ \\
\hline 78028 & & 8.64 & 0.02 & 0.63 & 0.02 & 1.54 & 0.03 & 18.30 & 0.81 & 4.95 & 0.08 & 1 & $\ldots$ \\
\hline 78241 & 143291 & 8.01 & 0.02 & 0.76 & 0.02 & 1.91 & 0.03 & 38.11 & 0.76 & 5.91 & 0.08 & 1 & $\ldots$ \\
\hline 78336 & 143295 & 9.09 & 0.02 & 0.88 & 0.04 & 2.25 & 0.03 & 30.27 & 1.10 & 6.49 & 0.14 & 1 & $\ldots$ \\
\hline 78550 & 143990 & 8.75 & 0.02 & 0.67 & 0.02 & 1.70 & 0.03 & 22.61 & 0.92 & 5.52 & 0.12 & 1 & $\ldots$ \\
\hline 78775 & 144579 & 6.65 & 0.02 & 0.74 & 0.02 & 1.90 & 0.03 & 68.88 & 0.33 & 5.84 & 0.13 & 1 & $\ldots$ \\
\hline 78923 & 144873 & 8.54 & 0.02 & 0.69 & 0.02 & 1.62 & 0.03 & 21.65 & 0.81 & 5.21 & 0.05 & 1 & $\ldots$ \\
\hline 79629 & 146868 & 7.69 & 0.02 & 0.65 & 0.02 & 1.69 & 0.03 & 32.54 & 0.44 & 5.25 & 0.13 & 2 & $\ldots$ \\
\hline 80243 & 148226 & 8.75 & 0.02 & 0.79 & 0.03 & 1.97 & 0.03 & 26.52 & 0.61 & 5.87 & 0.11 & 1 & $\ldots$ \\
\hline 80262 & 147750 & 8.44 & 0.02 & 0.72 & 0.02 & 1.74 & 0.03 & 25.20 & 0.92 & 5.44 & 0.06 & 1 & $\ldots$ \\
\hline 81186 & 149929 & 9.34 & 0.02 & 0.88 & 0.04 & 2.14 & 0.03 & 26.10 & 0.78 & 6.42 & 0.12 & 2 & $\ldots$ \\
\hline 81288 & 150172 & 8.95 & 0.02 & 0.88 & 0.03 & 2.29 & 0.03 & 32.19 & 0.90 & 6.49 & 0.08 & 1 & $\ldots$ \\
\hline 81605 & 150510 & 8.63 & 0.02 & 0.72 & 0.02 & 1.73 & 0.03 & 22.41 & 0.83 & 5.38 & 0.13 & 1 & $\ldots$ \\
\hline 81831 & $\ldots$ & 8.91 & 0.02 & 0.73 & 0.03 & 1.68 & 0.03 & 24.33 & 1.18 & 5.84 & 0.08 & 1 & $\ldots$ \\
\hline 82120 & 151192 & 8.23 & 0.02 & 0.68 & 0.02 & 1.71 & 0.03 & 27.40 & 1.02 & 5.42 & 0.05 & 2 & $\ldots$ \\
\hline 82210 & 152012 & 8.40 & 0.02 & 0.67 & 0.02 & 1.56 & 0.03 & 22.91 & 0.75 & 5.20 & 0.06 & 1 & $\ldots$ \\
\hline 82388 & 151798 & 7.95 & 0.02 & 0.61 & 0.02 & 1.47 & 0.03 & 23.78 & 0.85 & 4.83 & 0.13 & 2 & $\ldots$ \\
\hline 82644 & 152793 & 8.68 & 0.02 & 0.64 & 0.02 & 1.56 & 0.03 & 19.65 & 0.67 & 5.15 & 0.08 & 2 & $\ldots$ \\
\hline 82712 & $\ldots$ & 8.52 & 0.02 & 0.70 & 0.02 & 1.78 & 0.03 & 25.44 & 0.95 & 5.55 & 0.10 & 1 & $\ldots$ \\
\hline 83141 & $\ldots$ & 9.37 & 0.02 & 0.96 & 0.04 & 2.36 & 0.03 & 28.55 & 0.97 & 6.65 & 0.15 & 2 & $\ldots$ \\
\hline 83276 & 153631 & 7.12 & 0.02 & 0.62 & 0.02 & 1.54 & 0.03 & 36.52 & 1.36 & 4.93 & 0.05 & 2 & $\ldots$ \\
\hline 83500 & $\ldots$ & 8.82 & 0.02 & 0.62 & 0.03 & 1.46 & 0.03 & 16.29 & 0.81 & 4.88 & 0.04 & 1 & $\ldots$ \\
\hline 84520 & 156728 & 8.03 & 0.02 & 0.64 & 0.02 & 1.53 & 0.03 & 24.85 & 0.59 & 5.01 & 0.04 & 1 & $\ldots$ \\
\hline 85235 & 158633 & 6.43 & 0.02 & 0.76 & 0.02 & 1.92 & 0.03 & 78.11 & 0.30 & 5.89 & 0.14 & 2 & $\ldots$ \\
\hline 85653 & 159062 & 7.22 & 0.02 & 0.74 & 0.02 & 1.83 & 0.03 & 44.91 & 0.50 & 5.48 & 0.15 & 1 & $\ldots$ \\
\hline 86568 & $\ldots$ & 9.75 & 0.03 & 0.74 & 0.05 & 1.91 & 0.03 & 17.12 & 1.01 & 5.92 & 0.14 & 2 & $\ldots$ \\
\hline 86765 & 161098 & 7.67 & 0.02 & 0.67 & 0.02 & 1.66 & 0.04 & 33.14 & 0.75 & 5.27 & 0.02 & 1 & $\ldots$ \\
\hline 87089 & 161848 & 8.91 & 0.02 & 0.80 & 0.03 & 2.09 & 0.03 & 26.29 & 1.03 & 6.01 & 0.04 & 1 & $\ldots$ \\
\hline 88166 & $\ldots$ & 9.22 & 0.02 & 0.70 & 0.03 & 1.79 & 0.03 & 20.52 & 1.33 & 5.78 & 0.12 & 2 & $\ldots$ \\
\hline 88972 & 166620 & 6.40 & 0.02 & 0.87 & 0.02 & 2.17 & 0.03 & 90.71 & 0.30 & 6.19 & 0.15 & 1 & $\ldots$ \\
\hline $90355^{\mathrm{c}}$ & 169822 & 7.83 & 0.02 & 0.69 & 0.02 & 1.75 & 0.03 & 34.63 & 1.39 & 5.53 & 0.11 & 1 & $\ldots$ \\
\hline 90415 & $\ldots$ & 9.88 & 0.03 & 0.91 & 0.05 & 2.16 & 0.03 & 20.56 & 0.99 & 6.44 & 0.10 & 3 & $\ldots$ \\
\hline 90708 & $\ldots$ & 9.13 & 0.02 & 0.64 & 0.03 & 1.54 & 0.03 & 15.31 & 0.83 & 5.06 & 0.08 & 1 & $\ldots$ \\
\hline 91364 & 172393 & 8.33 & 0.02 & 0.84 & 0.02 & 2.05 & 0.03 & 35.60 & 0.66 & 6.09 & 0.06 & 1 & $\ldots$ \\
\hline 91381 & 172310 & 8.40 & 0.02 & 0.71 & 0.02 & 1.76 & 0.03 & 26.86 & 1.03 & 5.54 & 0.12 & 1 & $\ldots$ \\
\hline 91438 & 172051 & 5.86 & 0.02 & 0.66 & 0.02 & $\ldots$ & $\ldots$ & 76.42 & 0.46 & 5.28 & 0.08 & $\ldots$ & 1 \\
\hline 91605 & $\ldots$ & 8.57 & 0.02 & 0.93 & 0.03 & 2.35 & 0.03 & 42.48 & 1.11 & 6.71 & 0.05 & 1 & $\ldots$ \\
\hline 91614 & 336669 & 8.99 & 0.02 & 0.69 & 0.03 & 1.62 & 0.03 & 18.00 & 0.98 & 5.27 & 0.06 & 1 & $\ldots$ \\
\hline 91905 & 173665 & 8.54 & 0.02 & 0.75 & 0.03 & 1.81 & 0.03 & 27.81 & 0.54 & 5.76 & 0.09 & 2 & $\ldots$ \\
\hline 92388 & 229635 & 8.58 & 0.02 & 0.72 & 0.03 & 1.86 & 0.03 & 27.29 & 0.83 & 5.76 & 0.06 & 1 & $\ldots$ \\
\hline 92569 & 174719 & 7.51 & 0.02 & 0.68 & 0.02 & 1.66 & 0.03 & 35.20 & 0.62 & 5.24 & 0.06 & 1 & $\ldots$ \\
\hline 93195 & 176157 & 8.37 & 0.02 & 0.82 & 0.02 & 1.98 & 0.03 & 32.41 & 0.97 & 5.92 & 0.10 & $\ldots$ & 1 \\
\hline 93731 & 177745 & 8.54 & 0.02 & 0.79 & 0.02 & 1.92 & 0.03 & 31.47 & 1.01 & 6.03 & 0.09 & 1 & $\ldots$ \\
\hline 94582 & $\ldots$ & 9.54 & 0.02 & 0.78 & 0.04 & 1.83 & 0.03 & 18.09 & 0.68 & 5.82 & 0.14 & 2 & $\ldots$ \\
\hline 95727 & 231510 & 9.01 & 0.02 & 0.77 & 0.03 & 1.89 & 0.03 & 23.76 & 1.46 & 5.89 & 0.05 & 3 & $\ldots$ \\
\hline 96100 & 185144 & 4.69 & 0.02 & 0.80 & 0.02 & $\ldots$ & $\ldots$ & 173.77 & 0.18 & 5.89 & 0.13 & 1 & $\ldots$ \\
\hline 96344 & $\ldots$ & 9.75 & 0.03 & 0.66 & 0.05 & 1.52 & 0.03 & 12.52 & 0.75 & 5.24 & 0.12 & 2 & $\ldots$ \\
\hline 96634 & $\ldots$ & 9.13 & 0.02 & 0.79 & 0.03 & 2.01 & 0.03 & 21.89 & 0.84 & 5.83 & 0.11 & 2 & $\ldots$ \\
\hline 96735 & $\ldots$ & 9.20 & 0.02 & 0.87 & 0.03 & 2.21 & 0.03 & 26.09 & 0.91 & 6.28 & 0.12 & 2 & $\ldots$ \\
\hline $97640^{\mathrm{c}}$ & 226099 & 8.02 & 0.02 & 0.78 & 0.02 & 2.14 & 0.03 & 35.20 & 0.61 & 5.75 & 0.13 & 1 & $\ldots$ \\
\hline 97668 & 187645 & 9.58 & 0.05 & 0.90 & 0.08 & 2.26 & 0.05 & 26.95 & 1.85 & 6.74 & 0.11 & $\ldots$ & 2 \\
\hline $98288^{c}$ & 190061 & 9.27 & 0.02 & 0.62 & 0.03 & 1.58 & 0.03 & 13.26 & 0.68 & 4.88 & 0.07 & 2 & $\ldots$ \\
\hline
\end{tabular}


Table 1

(Continued)

\begin{tabular}{|c|c|c|c|c|c|c|c|c|c|c|c|c|c|}
\hline \multirow[t]{2}{*}{ HIP } & \multirow[t]{2}{*}{ HD } & \multirow{2}{*}{$\begin{array}{c}V \\
(\mathrm{mag})\end{array}$} & \multirow{2}{*}{$\begin{array}{l}\sigma(V) \\
(\mathrm{mag})\end{array}$} & \multirow{2}{*}{$\begin{array}{l}B-V \\
(\mathrm{mag})\end{array}$} & \multirow{2}{*}{$\begin{array}{l}\sigma_{B}-V \\
(\mathrm{mag})\end{array}$} & \multirow{2}{*}{$\begin{array}{l}V-K_{s} \\
(\mathrm{mag})\end{array}$} & \multirow{2}{*}{$\begin{array}{l}\sigma_{V-K_{S}} \\
(\mathrm{mag})\end{array}$} & \multirow{2}{*}{$\begin{array}{c}\pi^{\mathrm{a}} \\
(\operatorname{arcsec})\end{array}$} & \multirow{2}{*}{$\begin{array}{c}\sigma_{\pi}^{\mathrm{a}} \\
(\operatorname{arcsec})\end{array}$} & \multirow{2}{*}{$\begin{array}{c}M_{V}^{\mathrm{b}} \\
\text { (mag) }\end{array}$} & \multirow{2}{*}{$\begin{array}{c}\sigma\left(M_{V}\right) \\
(\mathrm{mag})\end{array}$} & \multicolumn{2}{|c|}{ Number of Obs. } \\
\hline & & & & & & & & & & & & Spring & Autumn \\
\hline 98413 & 189242 & 9.23 & 0.02 & 0.90 & 0.03 & 2.26 & 0.03 & 27.32 & 1.43 & 6.42 & 0.04 & $\ldots$ & 2 \\
\hline 98559 & 189540 & 9.30 & 0.02 & 0.70 & 0.03 & 1.72 & 0.03 & 17.12 & 1.10 & 5.47 & 0.11 & $\ldots$ & 2 \\
\hline 98677 & 190067 & 7.15 & 0.02 & 0.74 & 0.02 & 1.83 & 0.03 & 52.70 & 0.65 & 5.76 & 0.15 & 1 & $\ldots$ \\
\hline 98792 & 190404 & 7.27 & 0.02 & 0.84 & 0.02 & 2.16 & 0.03 & 63.42 & 0.57 & 6.28 & 0.02 & 1 & 2 \\
\hline 98965 & 190609 & 8.63 & 0.02 & 0.72 & 0.02 & 1.75 & 0.03 & 25.04 & 1.29 & 5.63 & 0.05 & 1 & $\ldots$ \\
\hline 99355 & 192139 & 8.74 & 0.02 & 0.89 & 0.03 & 2.21 & 0.03 & 35.68 & 0.83 & 6.50 & 0.09 & 1 & $\ldots$ \\
\hline 99774 & & 9.12 & 0.02 & 0.60 & 0.03 & 1.59 & 0.03 & 17.01 & 0.65 & 5.28 & 0.14 & 2 & $\ldots$ \\
\hline $99965^{\mathrm{c}}$ & 193216 & 8.16 & 0.02 & 0.76 & 0.02 & 1.75 & 0.03 & 32.51 & 0.55 & 5.72 & 0.12 & 1 & $\ldots$ \\
\hline 100259 & 193554 & 8.27 & 0.02 & 0.62 & 0.02 & 1.60 & 0.03 & 21.64 & 0.77 & 4.94 & 0.03 & 1 & $\ldots$ \\
\hline 101579 & 196124 & 8.94 & 0.02 & 0.95 & 0.03 & 2.41 & 0.03 & 34.91 & 1.20 & 6.65 & 0.03 & $\ldots$ & 2 \\
\hline 101997 & 196761 & 6.37 & 0.02 & 0.72 & 0.02 & $\ldots$ & $\ldots$ & 69.54 & 0.40 & 5.58 & 0.13 & $\ldots$ & 1 \\
\hline 102290 & 197300 & 8.65 & 0.02 & 0.60 & 0.03 & 1.45 & 0.03 & 17.54 & 0.96 & 4.87 & 0.10 & $\ldots$ & 2 \\
\hline 102521 & 197818 & 7.66 & 0.02 & 0.61 & 0.02 & 1.51 & 0.03 & 27.83 & 0.78 & 4.88 & 0.04 & $\ldots$ & 1 \\
\hline 103611 & 199803 & 8.57 & 0.02 & 0.68 & 0.03 & 1.61 & 0.03 & 22.15 & 1.03 & 5.29 & 0.12 & 2 & $\ldots$ \\
\hline 103895 & & 9.17 & 0.02 & 0.69 & 0.03 & 1.77 & 0.03 & 19.70 & 0.95 & 5.64 & 0.15 & 2 & $\ldots$ \\
\hline 103896 & 200297 & 8.55 & 0.02 & 0.63 & 0.03 & 1.56 & 0.03 & 21.42 & 1.10 & 5.21 & 0.12 & $\ldots$ & 2 \\
\hline 104375 & 202123 & 8.68 & 0.02 & 0.87 & 0.03 & 2.10 & 0.03 & 32.10 & 0.63 & 6.21 & 0.13 & 1 & $\ldots$ \\
\hline 104733 & 202108 & 7.33 & 0.02 & 0.65 & 0.02 & 1.58 & 0.03 & 37.13 & 0.56 & 5.18 & 0.03 & 1 & 1 \\
\hline 106231 & & 9.25 & 0.02 & 0.99 & 0.04 & 2.86 & 0.03 & 40.32 & 1.06 & 7.27 & 0.07 & $\ldots$ & 2 \\
\hline 106949 & & 8.97 & 0.02 & 0.65 & 0.02 & 1.66 & 0.03 & 18.89 & 1.00 & 5.36 & 0.11 & 2 & $\ldots$ \\
\hline 107038 & 207897 & 8.40 & 0.02 & 0.87 & 0.02 & 2.09 & 0.03 & 35.49 & 0.48 & 6.15 & 0.05 & 1 & $\ldots$ \\
\hline 107700 & 208002 & 8.98 & 0.02 & 0.86 & 0.03 & 2.12 & 0.03 & 27.20 & 0.78 & 6.15 & 0.08 & 1 & $\ldots$ \\
\hline 108774 & 209393 & 7.94 & 0.02 & 0.70 & 0.02 & 1.62 & 0.03 & 29.61 & 0.82 & 5.30 & 0.05 & 1 & $\ldots$ \\
\hline 108947 & 209599 & 8.39 & 0.02 & 0.79 & 0.03 & 1.93 & 0.03 & 31.24 & 0.96 & 5.86 & 0.07 & 1 & $\ldots$ \\
\hline 109310 & 210323 & 8.41 & 0.02 & 0.62 & 0.02 & 1.45 & 0.03 & 19.69 & 0.97 & 4.88 & 0.07 & $\ldots$ & 2 \\
\hline 110508 & 212291 & 7.91 & 0.02 & 0.70 & 0.02 & 1.64 & 0.03 & 30.07 & 0.60 & 5.30 & 0.06 & 1 & $\ldots$ \\
\hline 111883 & & 8.76 & 0.02 & 0.89 & 0.03 & 2.18 & 0.03 & 32.84 & 0.97 & 6.34 & 0.09 & $\ldots$ & 2 \\
\hline 111888 & 214683 & 8.48 & 0.02 & 0.90 & 0.03 & 2.34 & 0.03 & 41.51 & 0.77 & 6.57 & 0.15 & 1 & $\ldots$ \\
\hline 111977 & 215065 & 7.46 & 0.02 & 0.63 & 0.02 & 1.61 & 0.03 & 34.10 & 0.47 & 5.12 & 0.14 & 1 & $\ldots$ \\
\hline 112245 & 215500 & 7.50 & 0.02 & 0.73 & 0.02 & 1.77 & 0.03 & 39.76 & 0.56 & 5.50 & 0.05 & 1 & $\ldots$ \\
\hline 112870 & 216259 & 8.29 & 0.02 & 0.87 & 0.02 & 2.21 & 0.03 & 46.97 & 1.01 & 6.65 & 0.09 & $\ldots$ & 1 \\
\hline 113231 & 216777 & 8.00 & 0.02 & 0.65 & 0.02 & 1.64 & 0.03 & 27.21 & 1.12 & 5.17 & 0.03 & 1 & $\ldots$ \\
\hline 113884 & 217924 & 7.22 & 0.02 & 0.62 & 0.02 & 1.57 & 0.03 & 38.37 & 2.11 & 5.14 & 0.05 & 1 & 2 \\
\hline 113989 & 218209 & 7.50 & 0.02 & 0.64 & 0.02 & 1.70 & 0.03 & 33.84 & 0.38 & 5.15 & 0.06 & 1 & $\ldots$ \\
\hline 114340 & 218614 & 8.78 & 0.02 & 0.67 & 0.03 & 1.57 & 0.03 & 19.23 & 1.09 & 5.20 & 0.08 & $\ldots$ & 2 \\
\hline 114385 & 218739 & 7.14 & 0.02 & 0.65 & 0.02 & 1.47 & 0.03 & 40.08 & 2.19 & 5.15 & 0.06 & $\ldots$ & 1 \\
\hline 115194 & 219953 & 8.86 & 0.02 & 0.79 & 0.03 & 2.11 & 0.03 & 30.20 & 1.04 & 6.26 & 0.09 & $\ldots$ & 2 \\
\hline 115411 & 220293 & 8.74 & 0.02 & 0.67 & 0.03 & 1.85 & 0.03 & 22.58 & 1.04 & 5.51 & 0.08 & $\ldots$ & 1 \\
\hline 115445 & 220339 & 7.79 & 0.02 & 0.90 & 0.03 & 2.19 & 0.03 & 52.27 & 0.86 & 6.38 & 0.03 & $\ldots$ & 1 \\
\hline 116005 & 221239 & 8.35 & 0.02 & 0.88 & 0.02 & 2.21 & 0.03 & 38.64 & 0.86 & 6.29 & 0.04 & $\ldots$ & 3 \\
\hline 116410 & 221822 & 8.40 & 0.02 & 0.74 & 0.03 & 1.76 & 0.03 & 26.87 & 1.35 & 5.54 & 0.14 & $\ldots$ & 1 \\
\hline 118207 & 224540 & 8.37 & 0.02 & 0.64 & 0.02 & 1.55 & 0.03 & 22.83 & 0.58 & 5.16 & 0.13 & $\ldots$ & 2 \\
\hline 118251 & & 8.15 & 0.02 & 0.64 & 0.02 & 1.57 & 0.03 & 23.86 & 0.65 & 5.04 & 0.09 & $\ldots$ & 1 \\
\hline 118278 & 224619 & 7.48 & 0.02 & 0.74 & 0.02 & 1.77 & 0.04 & 39.84 & 0.78 & 5.48 & 0.07 & $\ldots$ & 1 \\
\hline
\end{tabular}

Notes.

${ }^{a}$ Parallaxes and parallax errors from the revised Hipparcos catalog (van Leeuwen 2007a, 2007b).

b Absolute magnitude computed from the Hipparcos parallax.

${ }^{\mathrm{c}}$ A spectroscopic binary listed in the ninth catalog of spectroscopic binary orbits (Pourbaix et al. 2004, http://sb9.astro.ulb.ac.be).

is a corrected $[\mathrm{Fe} / \mathrm{H}]$ value, which was put on the metallicity scale adopted in this paper and is described below in detail. We computed an error in $[\mathrm{Fe} / \mathrm{H}]$ by propagating errors in $\mathrm{Fe}$ I and $\mathrm{Fe}$ II abundances. If repeat measurements $\left(N_{\mathrm{obs}}\right)$ exist, we took a standard deviation of these measurements divided by a square root of $N_{\mathrm{obs}}$ or the one propagated from individual abundance errors, whichever is larger. A typical root-mean-square (rms) dispersion of $\mathrm{Fe}$ I abundance is approximately $0.15 \mathrm{dex}$ for each star. Given the large number of iron lines used in this study $(N=37)$, this results in an unrealistically small error in $[\mathrm{Fe} / \mathrm{H}]$ ( $\sigma \approx 0.02 \mathrm{dex}$ ). To estimate more realistic errors in abundance, we ran additional models with different input stellar parameters
$\left(\Delta T_{\text {eff }}= \pm 100 \mathrm{~K}, \Delta \log g= \pm 0.3 \mathrm{dex}, \Delta[\mathrm{Fe} / \mathrm{H}]= \pm 0.1 \mathrm{dex}\right.$, and $\Delta \xi$ by $\pm 0.3 \mathrm{~km} \mathrm{~s}^{-1}$ ). Table 4 shows the mean abundance errors obtained from all stars analyzed using MOOG. A quadrature sum of these errors yields a systematic error in $[\mathrm{Fe} / \mathrm{H}]$ of the order of $0.08 \mathrm{dex}$.

For an external check on our derived stellar parameters, we compared our values with atmospheric parameters for a large number of field dwarfs in Valenti \& Fischer (2005, hereafter VF05). They used a software package Spectroscopy Made Easy (Valenti \& Piskunov 1996) to derive $T_{\text {eff }}, \log g$, and individual elemental abundances based on high-resolution spectra. They obtained both an overall metallicity $([\mathrm{M} / \mathrm{H}])$ 
Table 2

Atomic Line List

\begin{tabular}{|c|c|c|c|c|}
\hline Element & Ion & $\begin{array}{c}\text { Wavelength } \\
(\AA)\end{array}$ & $\begin{array}{c}\mathrm{EP} \\
(\mathrm{eV})\end{array}$ & $\log g f$ \\
\hline $\mathrm{Fe}$ & 1 & 5775.08 & 4.22 & -1.30 \\
\hline $\mathrm{Fe}$ & 1 & 5809.22 & 3.88 & -1.74 \\
\hline $\mathrm{Fe}$ & 1 & 5849.69 & 3.69 & -2.89 \\
\hline $\mathrm{Fe}$ & 1 & 5852.22 & 4.55 & -1.23 \\
\hline $\mathrm{Fe}$ & 1 & 5855.08 & 4.61 & -1.48 \\
\hline $\mathrm{Fe}$ & 1 & 5856.09 & 4.29 & -1.33 \\
\hline $\mathrm{Fe}$ & 1 & 5858.78 & 4.22 & -2.16 \\
\hline $\mathrm{Fe}$ & 1 & 5859.59 & 4.55 & -0.30 \\
\hline $\mathrm{Fe}$ & 1 & 5861.11 & 4.28 & -2.45 \\
\hline $\mathrm{Fe}$ & 1 & 5862.36 & 4.55 & -0.06 \\
\hline $\mathrm{Fe}$ & 1 & 6027.05 & 4.08 & -1.09 \\
\hline $\mathrm{Fe}$ & 1 & 6127.90 & 4.14 & -1.40 \\
\hline $\mathrm{Fe}$ & 1 & 6151.62 & 2.18 & -3.27 \\
\hline $\mathrm{Fe}$ & 1 & 6157.73 & 4.08 & -1.16 \\
\hline $\mathrm{Fe}$ & 1 & 6159.38 & 4.61 & -1.97 \\
\hline $\mathrm{Fe}$ & 1 & 6165.37 & 4.14 & -1.47 \\
\hline $\mathrm{Fe}$ & 1 & 6173.34 & 2.22 & -2.88 \\
\hline $\mathrm{Fe}$ & 1 & 6180.20 & 2.73 & -2.59 \\
\hline $\mathrm{Fe}$ & 1 & 6213.44 & 2.22 & -2.48 \\
\hline $\mathrm{Fe}$ & 1 & 6219.28 & 2.20 & -2.42 \\
\hline $\mathrm{Fe}$ & 1 & 6226.74 & 3.88 & -2.12 \\
\hline $\mathrm{Fe}$ & 1 & 6229.23 & 2.84 & -2.87 \\
\hline $\mathrm{Fe}$ & 1 & 6240.65 & 2.22 & -3.17 \\
\hline $\mathrm{Fe}$ & 1 & 6270.23 & 2.86 & -2.61 \\
\hline $\mathrm{Fe}$ & 1 & 6322.69 & 2.59 & -2.43 \\
\hline $\mathrm{Fe}$ & 1 & 6335.34 & 2.20 & -2.18 \\
\hline $\mathrm{Fe}$ & 1 & 6344.15 & 2.43 & -2.92 \\
\hline $\mathrm{Fe}$ & 1 & 6380.75 & 4.19 & -1.38 \\
\hline $\mathrm{Fe}$ & 1 & 6481.88 & 2.28 & -2.96 \\
\hline $\mathrm{Fe}$ & 1 & 6498.94 & 0.96 & -4.70 \\
\hline $\mathrm{Fe}$ & 1 & 6627.56 & 4.55 & -1.58 \\
\hline $\mathrm{Fe}$ & 1 & 6703.57 & 2.76 & -3.06 \\
\hline $\mathrm{Fe}$ & 1 & 6750.15 & 2.42 & -2.62 \\
\hline $\mathrm{Fe}$ & 1 & 7107.46 & 4.19 & -1.34 \\
\hline $\mathrm{Fe}$ & 1 & 7127.57 & 4.99 & -1.36 \\
\hline $\mathrm{Fe}$ & 1 & 7130.92 & 4.22 & -0.69 \\
\hline $\mathrm{Fe}$ & 1 & 7132.99 & 4.08 & -1.63 \\
\hline $\mathrm{Fe}$ & 2 & 6149.23 & 3.89 & -2.84 \\
\hline $\mathrm{Fe}$ & 2 & 6247.55 & 3.89 & -2.43 \\
\hline $\mathrm{Fe}$ & 2 & 6456.39 & 3.90 & -2.19 \\
\hline $\mathrm{Fe}$ & 2 & 6516.08 & 2.89 & -3.43 \\
\hline $\mathrm{Fe}$ & 2 & 7224.46 & 3.89 & -3.36 \\
\hline
\end{tabular}

and an elemental abundance of iron $([\mathrm{Fe} / \mathrm{H}])$ for each star, but we utilized their $[\mathrm{Fe} / \mathrm{H}]$ as we derived metallicities using MOOG from EW measurements of Fe.

Figure 2 shows comparisons in $T_{\text {eff }}$ (top), $\log g$ (middle), and $[\mathrm{Fe} / \mathrm{H}]$ (bottom) between MOOG and VF05. Statistical properties of the parameter comparisons are summarized in the first row of Table 5 . The MOOG $T_{\text {eff }}$ is on average $53 \mathrm{~K}$ higher than VF05 $T_{\text {eff }}$, which is not unexpected from the two independent analyses. However, our spectroscopic $\log g$ estimates are systematically smaller than those from VF05. The observed trend in $\log g$ is similar to those seen from a large number of disk stars in Bensby et al. (2014), who found that $\log g$ determinations from $\mathrm{Fe}_{\mathrm{I}}$ and $\mathrm{Fe}$ II ionization equilibrium are systematically smaller than the one based on the Hipparcos parallax. The offset was seen for dwarfs over a wide range of temperature in their study, but there was no convincing trend observed for giants. Theoretical models (An et al. 2007b, 2015) also suggest that MS stars within a narrow range of color $(0.6 \leqslant B-V \leqslant 1.0)$ have surface gravities $4.4 \lesssim \log g \lesssim 4.6$ over a range of metallicity covered by our sample stars, but our MOOG estimates are about $0.3 \mathrm{dex}$ smaller than these. Nevertheless, the $\log g$ dependence of our metallicity estimate from MOOG is weak (Table 4), and an adjustment of $\log g$ would hardly affect our $[\mathrm{Fe} / \mathrm{H}]$ estimates.

In Figure 2 observed $1 \sigma$ dispersions in the parameter comparisons are $\sigma\left(\Delta T_{\text {eff }}\right)=104 \mathrm{~K}$ and $\sigma(\Delta[\mathrm{Fe} / \mathrm{H}])=0.06$. VF05 computed formal $1 \sigma$ uncertainties of their parameter estimates as $\sigma\left(T_{\text {eff }}\right)=44 \mathrm{~K}$ and $\sigma([\mathrm{Fe} / \mathrm{H}])=0.03$, suggesting that $T_{\text {eff }}$ and $[\mathrm{Fe} / \mathrm{H}]$ determined from our MOOG analysis have a precision of $\sigma\left(T_{\text {eff }}\right) \approx 90 \mathrm{~K}$ and $\sigma([\mathrm{Fe} / \mathrm{H}]) \approx 0.05$ dex. The latter is close to our expectation $(\sigma \approx 0.08 \mathrm{dex})$ from errors computed by varying stellar input parameters in the models (Table 4).

We also checked the accuracy of stellar parameters derived using MOOG against a calibration sample in Casagrande et al. (2010), which has been used in the derivation of their empirical color $-T_{\text {eff }}$ relations based on the Infrared Flux Method (IRFM). They provided a large compilation of stellar parameters from a high-resolution spectroscopy in the literature. We crossidentified stars in their and our catalogs based on coordinates and $V$ magnitudes. There were nine stars in common, and the comparison with their stellar parameters is included in Table 5. The average difference in $T_{\text {eff }}$ between Casagrande et al. (2010) and our MOOG-based estimates is $32 \mathrm{~K}$.

Our $[\mathrm{Fe} / \mathrm{H}]$ estimates from MOOG are systematically lower than those in VF05 and in Casagrande et al. (2010) by $0.07 \pm 0.01$ dex and $0.02 \pm 0.01$ dex, respectively. However, the above metallicity estimates from MOOG were not adjusted for an instrumental correction to the solar $\mathrm{Fe}$ abundance, because we did not obtain a solar spectrum during the observing runs. As such, we adjusted our original $[\mathrm{Fe} / \mathrm{H}]$ estimates from MOOG to match published values in VF05 by adding a constant offset $(\Delta[\mathrm{Fe} / \mathrm{H}]=0.07 \mathrm{dex})$. The last column $\left([\mathrm{Fe} / \mathrm{H}]_{\text {corr }}\right)$ in Table 3 lists $[\mathrm{Fe} / \mathrm{H}]$ values after this correction.

\subsection{Spectral Matching Technique (SMT)}

In addition to the EW analysis using MOOG, we employed an SMT to derive stellar parameters $\left(T_{\mathrm{eff}}, \log g\right.$, and $\left.[\mathrm{Fe} / \mathrm{H}]\right)$ for all of our sample stars. The SMT routine is essentially a modified version of the NGS1 technique adopted in the SEGUE (Sloan Extension for Galactic Understanding and Exploration; Yanny et al. 2009) Stellar Parameter Pipeline (SSPP; Lee et al. 2008a, 2008b). It was originally designed to match a grid of synthetic models to low-resolution stellar spectra in the Sloan Digital Sky Survey (SDSS; York et al. 2000; Stoughton et al. 2002). We modified and optimized the original NGS1 code to analyze our echelle spectra. We found that the accuracy of stellar parameters derived from SMT remains almost unaffected with varying spectral resolutions. For this reason, we linearly re-binned observed spectra with Gaussian smoothing to $0.25 \AA$ per pixel $(R \approx 10,000)$ for a fast and efficient analysis of our extensive data set. The smoothing was also helpful for improving the $\mathrm{S} / \mathrm{N}$ of the spectra.

We generated a grid of models using the Kurucz stellar model atmospheres based on the new ODFs as described previously. We utilized a pre-computed set of models ${ }^{13}$ to construct a

\footnotetext{
${ }^{13}$ Available at http://kurucz.harvard.edu/grids.html.
} 
Table 3

Stellar Parameters derived using MOOG

\begin{tabular}{|c|c|c|c|c|c|c|}
\hline HIP & $\begin{array}{l}T_{\text {eff }} \\
(\mathrm{K})\end{array}$ & $\log g$ & {$[\mathrm{Fe} / \mathrm{H}]$} & $\begin{array}{c}\xi \\
\left(\mathrm{km} \mathrm{s}^{-1}\right)\end{array}$ & {$[\mathrm{Fe} / \mathrm{H}]_{\text {corr }}{ }^{\mathrm{a}}$} & $\sigma([\mathrm{Fe} / \mathrm{H}])$ \\
\hline 4907 & 5405 & 4.34 & -0.16 & 1.2 & -0.09 & 0.019 \\
\hline $5313^{\mathrm{b}}$ & 5881 & 4.05 & -0.29 & 1.3 & -0.22 & 0.017 \\
\hline 5521 & 5398 & 4.00 & -0.29 & 1.1 & -0.22 & 0.009 \\
\hline 9172 & 5638 & 4.04 & -0.13 & 1.4 & -0.06 & 0.009 \\
\hline 9829 & 5795 & 4.29 & -0.22 & 1.1 & -0.14 & 0.020 \\
\hline $10276^{\mathrm{b}}$ & 5368 & 4.42 & -0.25 & 1.2 & -0.18 & 0.016 \\
\hline 15442 & 5760 & 4.18 & -0.27 & 1.3 & -0.20 & 0.026 \\
\hline 17265 & 5339 & 4.23 & -0.69 & 1.3 & -0.62 & 0.026 \\
\hline 18324 & 5216 & 4.01 & -0.55 & 1.4 & -0.48 & 0.030 \\
\hline 21276 & 5448 & 4.27 & -0.17 & 1.4 & -0.10 & 0.024 \\
\hline 21571 & 5636 & 4.36 & -0.30 & 1.3 & -0.23 & 0.016 \\
\hline 21832 & 5609 & 4.17 & -0.67 & 1.3 & -0.60 & 0.026 \\
\hline 22175 & 5765 & 4.28 & -0.16 & 1.4 & -0.09 & 0.025 \\
\hline 23786 & 5261 & 4.13 & -0.27 & 1.2 & -0.20 & 0.023 \\
\hline 26505 & 4948 & 4.10 & -0.60 & 1.0 & -0.53 & 0.015 \\
\hline 30862 & 5663 & 4.15 & -0.56 & 1.2 & -0.49 & 0.010 \\
\hline 52470 & 5367 & 4.34 & -0.46 & 1.1 & -0.39 & 0.028 \\
\hline 54906 & 5403 & 4.31 & -0.26 & 1.3 & -0.19 & 0.032 \\
\hline 55210 & 5314 & 3.81 & -0.36 & 1.1 & -0.28 & 0.018 \\
\hline $56092^{\mathrm{b}}$ & 5030 & 4.33 & -0.44 & 1.2 & -0.37 & 0.020 \\
\hline 57939 & 5072 & 3.77 & -1.42 & 1.1 & -1.35 & 0.021 \\
\hline 57992 & 5431 & 4.36 & -0.43 & 1.0 & -0.36 & 0.030 \\
\hline 58949 & 5291 & 4.14 & -0.32 & 0.9 & -0.25 & 0.024 \\
\hline 60074 & 5915 & 3.92 & -0.12 & 1.6 & -0.05 & 0.019 \\
\hline 60268 & 5678 & 3.98 & -0.60 & 0.8 & -0.53 & 0.031 \\
\hline 63346 & 5779 & 4.36 & -0.45 & 1.6 & -0.38 & 0.031 \\
\hline 63636 & 5739 & 3.90 & -0.25 & 1.7 & -0.18 & 0.028 \\
\hline 65165 & 5431 & 4.13 & -0.13 & 1.2 & -0.06 & 0.026 \\
\hline 65352 & 5178 & 3.99 & -0.48 & 1.1 & -0.40 & 0.018 \\
\hline 65355 & 5054 & 4.13 & -0.42 & 1.0 & -0.35 & 0.026 \\
\hline 67904 & 5304 & 4.37 & -0.44 & 1.1 & -0.37 & 0.014 \\
\hline 70253 & 5550 & 3.80 & -0.66 & 1.0 & -0.59 & 0.021 \\
\hline 70319 & 5608 & 3.88 & -0.46 & 1.2 & -0.39 & 0.014 \\
\hline $71720^{b}$ & 5285 & 4.50 & -0.24 & 1.2 & -0.17 & 0.029 \\
\hline 73005 & 5219 & 4.33 & -0.44 & 0.9 & -0.37 & 0.025 \\
\hline $73138^{b}$ & 5327 & 4.39 & -0.07 & 1.0 & +0.00 & 0.027 \\
\hline $73845^{\mathrm{b}}$ & 5620 & 3.50 & -0.60 & 0.8 & -0.53 & 0.025 \\
\hline $74126^{\mathrm{b}}$ & 5805 & 4.36 & -0.02 & 1.2 & +0.05 & 0.031 \\
\hline 74396 & 5166 & 4.44 & -0.23 & 1.2 & -0.16 & 0.027 \\
\hline $75446^{\mathrm{b}}$ & 5519 & 4.16 & -0.15 & 1.1 & -0.08 & 0.026 \\
\hline 76330 & 5101 & 4.15 & -0.31 & 1.5 & -0.24 & 0.029 \\
\hline $77810^{\mathrm{b}}$ & 5949 & 4.07 & +0.01 & 1.2 & +0.08 & 0.023 \\
\hline 78241 & 5318 & 3.86 & -0.46 & 1.1 & -0.39 & 0.029 \\
\hline $78336^{\mathrm{b}}$ & 5053 & 4.32 & -0.05 & 1.3 & +0.02 & 0.025 \\
\hline 78775 & 5233 & 3.87 & -0.76 & 1.0 & -0.69 & 0.021 \\
\hline 79629 & 5663 & 4.19 & -0.35 & 1.3 & -0.28 & 0.017 \\
\hline 80243 & 5356 & 4.14 & -0.10 & 1.5 & -0.03 & 0.026 \\
\hline 81186 & 5031 & 4.19 & -0.45 & 1.2 & -0.37 & 0.017 \\
\hline 81288 & 4934 & 4.33 & -0.91 & 0.8 & -0.84 & 0.019 \\
\hline $81831^{b}$ & 5509 & 4.16 & -0.37 & 1.2 & -0.30 & 0.025 \\
\hline 84520 & 5708 & 3.90 & -0.29 & 1.3 & -0.22 & 0.023 \\
\hline 85235 & 5263 & 4.21 & -0.51 & 1.0 & -0.43 & 0.015 \\
\hline 85653 & 5465 & 4.43 & -0.48 & 1.3 & -0.41 & 0.018 \\
\hline 86765 & 5625 & 4.21 & -0.27 & 1.1 & -0.20 & 0.025 \\
\hline 88972 & 5048 & 4.26 & -0.35 & 1.1 & -0.28 & 0.027 \\
\hline 91364 & 5268 & 4.11 & -0.43 & 1.3 & -0.36 & 0.028 \\
\hline 91438 & 5605 & 4.25 & -0.31 & 1.2 & -0.24 & 0.020 \\
\hline 91605 & 5026 & 4.04 & -0.52 & 1.3 & -0.45 & 0.026 \\
\hline 91905 & 5396 & 4.20 & -0.42 & 1.2 & -0.34 & 0.019 \\
\hline 92569 & 5651 & 3.29 & -0.27 & 1.3 & -0.20 & 0.025 \\
\hline 93195 & 5191 & 4.10 & -0.18 & 1.2 & -0.11 & 0.025 \\
\hline 96100 & 5438 & 4.25 & -0.28 & 1.4 & -0.21 & 0.026 \\
\hline $97668^{b}$ & 5016 & 4.41 & -0.29 & 1.2 & -0.22 & 0.021 \\
\hline 98677 & 5415 & 4.38 & -0.36 & 0.9 & -0.29 & 0.022 \\
\hline
\end{tabular}

Table 3

(Continued)

\begin{tabular}{lcccccc}
\hline \hline HIP & $\begin{array}{c}T_{\text {eff }} \\
(\mathrm{K})\end{array}$ & $\log g$ & {$[\mathrm{Fe} / \mathrm{H}]$} & $\begin{array}{c}\xi \\
\left(\mathrm{km} \mathrm{s}^{-1}\right)\end{array}$ & {$[\mathrm{Fe} / \mathrm{H}]_{\text {corr }}{ }^{\mathrm{a}}$} & $\sigma([\mathrm{Fe} / \mathrm{H}])$ \\
\hline 98792 & 5104 & 4.46 & -0.70 & 1.1 & -0.63 & 0.013 \\
99355 & 5227 & 4.27 & -0.49 & 1.7 & -0.42 & 0.029 \\
99965 & 5730 & 3.96 & +0.08 & 1.4 & +0.15 & 0.026 \\
101997 & 5511 & 4.24 & -0.30 & 1.1 & -0.23 & 0.027 \\
102521 & 5688 & 4.01 & -0.35 & 1.3 & -0.28 & 0.019 \\
104375 & 5117 & 4.38 & -0.41 & 0.9 & -0.34 & 0.024 \\
104733 & 5774 & 4.09 & -0.25 & 1.3 & -0.18 & 0.018 \\
107038 & 5277 & 4.28 & -0.22 & 1.4 & -0.15 & 0.028 \\
107700 & 5253 & 4.53 & -0.34 & 1.3 & -0.27 & 0.028 \\
108774 & 5653 & 4.06 & -0.25 & 1.4 & -0.18 & 0.026 \\
110508 & 5714 & 3.55 & -0.19 & 1.4 & -0.12 & 0.026 \\
111883 & 5021 & 4.36 & -0.44 & 1.2 & -0.37 & 0.013 \\
111888 & 4904 & 4.44 & -0.40 & 1.2 & -0.33 & 0.032 \\
111977 & 5686 & 4.27 & -0.54 & 1.3 & -0.47 & 0.024 \\
112245 & 5631 & 4.09 & -0.26 & 1.6 & -0.19 & 0.026 \\
112870 & 4930 & 4.30 & -0.74 & 0.8 & -0.67 & 0.026 \\
113989 & 5607 & 4.07 & -0.58 & 1.4 & -0.51 & 0.025 \\
$114385^{\mathrm{b}}$ & 5906 & 4.16 & +0.03 & 1.5 & +0.10 & 0.023 \\
115445 & 5115 & 4.33 & -0.40 & 1.3 & -0.33 & 0.028 \\
116005 & 5024 & 4.35 & -0.28 & 1.2 & -0.21 & 0.015 \\
118207 & 5700 & 4.32 & -0.33 & 1.1 & -0.25 & 0.012 \\
118251 & 5693 & 3.75 & -0.51 & 1.3 & -0.44 & 0.029 \\
118278 & 5604 & 3.87 & -0.21 & 1.4 & -0.14 & 0.031 \\
\hline
\end{tabular}

Notes.

${ }^{\text {a }}$ Corrected to match the metallicity scale of VF05 (see text).

${ }^{\mathrm{b}}$ Stars with less accurate parallax measurements $\left(\sigma_{\pi} / \pi>3 \%\right)$, but included as having larger magnitude excesses $\left(\Delta M_{V}\right)$ than the Pleiades.

Table 4

Systematic Errors in Abundance Measurements from MOOG Analysis

\begin{tabular}{lcccc}
\hline \hline Ion & $\Delta T_{\text {eff }}$ & $\Delta \log g$ & $\Delta[\mathrm{Fe} / \mathrm{H}]$ & \\
& $\pm 100 \mathrm{~K}$ & $\pm 0.3 \mathrm{dex}$ & $\pm 0.1 \mathrm{dex}$ & $\pm 0.2 \mathrm{~km} \mathrm{~s}^{-1}$ \\
\hline $\mathrm{Fe}_{\text {I }}$ & \pm 0.06 & $\mp 0.01$ & \pm 0.01 & $\mp 0.04$ \\
$\mathrm{Fe}_{\text {II }}$ & $\mp 0.06$ & \pm 0.14 & \pm 0.02 & $\mp 0.04$ \\
\hline
\end{tabular}

finer grid by linearly interpolating these models over a wide range of parameter space, covering $4000 \mathrm{~K}<T_{\text {eff }}<10000 \mathrm{~K}$ in steps of $250 \mathrm{~K}, 0.0<\log g<5.0$ in steps of $0.25 \mathrm{dex}$, and $-5.0<[\mathrm{Fe} / \mathrm{H}]<+1.0$ in steps of 0.25 dex. Synthetic model spectra were then generated using the synthe code at $3000 \AA$ $\leqslant \lambda \leqslant 10,000 \AA$ at a resolution of $0.01 \AA$, where we adopted $\xi\left[\mathrm{km} \mathrm{s}^{-1}\right]=-0.345 \times \log g+2.22$, which was derived from the SSPP calibration with high-resolution spectra. After constructing a full set of synthetic spectra, we re-sampled model spectra to $0.25 \AA$ wide linear pixels, corresponding to $R=10,000$ at $5000 \AA$.

We matched synthetic spectra with the observed data at $4500 \AA<\lambda<5500 \AA$ and $5900 \AA<\lambda<6900 \AA$, which contain a large number of isolated $\mathrm{Fe}$ lines along with other various metallic lines. In each of these wavelength ranges, we normalized model spectra using a pseudo-continuum that was constructed by iteratively rejecting data points more than $1 \sigma$ below and $4 \sigma$ above a fitted polynomial curve. The degree of a polynomial was set to 21 to cover each echelle order. We took the same normalization step for the observed spectra, which 

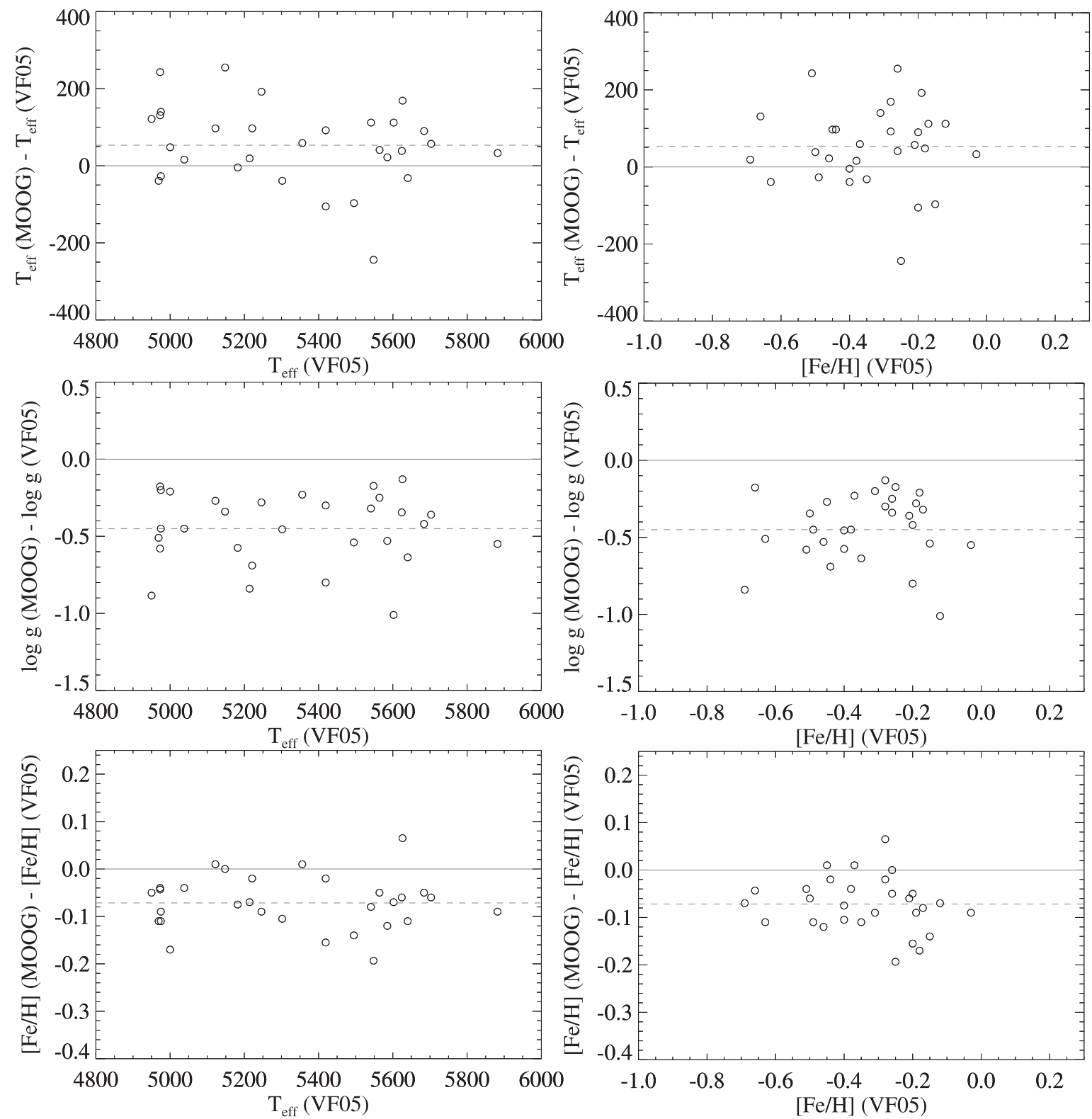

Figure 2. Comparisons in spectroscopic parameters between VF05 and MOOG. The dashed line indicates a mean difference, while the solid line is a zero difference. In the bottom panels, metallicities from MOOG represent raw estimates, before applying a zero-point adjustment (see text).

Table 5

Comparisons between Various Spectroscopic Parameter Estimates

\begin{tabular}{lcccr}
\hline \hline Comparison & $\left\langle\Delta T_{\text {eff }}\right\rangle$ & $\langle\Delta \log g\rangle$ & $\langle\Delta[\mathrm{Fe} / \mathrm{H}]\rangle$ & $N_{\text {star }}$ \\
& $(\mathrm{K})$ & & & \\
\hline MOOG - VF05 & $+53 \pm 10$ & $-0.45 \pm 0.08$ & $-0.07 \pm 0.01$ & 30 \\
MOOG - C10 & $+32 \pm 11$ & $-0.35 \pm 0.12$ & $-0.02 \pm 0.01$ & 9 \\
SMT3 - MOOG & $+37 \pm 4$ & $+0.35 \pm 0.04$ & $+0.16 \pm 0.02$ & 74 \\
SMT3 - SMT2 & $+51 \pm 4$ & $+0.09 \pm 0.01$ & $+0.03 \pm 0.00$ & 170 \\
SMT3 - VF05 & $+85 \pm 14$ & $-0.10 \pm 0.02$ & $+0.07 \pm 0.01$ & 36 \\
SMT3 - C10 & $+84 \pm 25$ & $-0.08 \pm 0.02$ & $+0.14 \pm 0.04$ & 11 \\
\hline
\end{tabular}

Note. All comparisons made using original metallicity estimates.

produced the same level of line-strength suppression. We used the $\chi^{2}$ minimization routine MPFIT ${ }^{14}$ (Markwardt 2009) to search the grid of synthetic spectra for the best-fitting model

$\overline{{ }^{14} \text { http://www.physics.wisc.edu/ craigm/idl/fitting.html }}$ parameters. In this step, we generated a synthetic spectrum at intermediate values of $T_{\text {eff }}, \log g$, and $[\mathrm{Fe} / \mathrm{H}]$ from the model grid using a spline interpolation. Errors in the best-fitting model parameters were determined by the square root of the diagonal elements in the covariance matrix.

Figure 3 shows an example of the results from SMT. The black solid line is an observed spectrum of HIP 98677 after downgrading its resolution to $R=10,000$. The red solid line represents our best-fitting synthetic spectrum with $T_{\text {eff }}=5519 \mathrm{~K}, \log g=4.56 \mathrm{dex},[\mathrm{Fe} / \mathrm{H}]=-0.24 \mathrm{dex}$, and $\xi=0.65 \mathrm{~km} \mathrm{~s}^{-1}$, which shows an excellent match to the observed data. The second through seventh columns in Table 6 summarize results from SMT with $1 \sigma$ errors in $T_{\text {eff }}, \log g$, and $[\mathrm{Fe} / \mathrm{H}]$. For stars observed multiple times, the average values of individual parameter estimates are listed. Errors in these quantities are either random errors derived from SMT or a standard deviation of measurements from multiple observations divided by a square root of $N_{\mathrm{obs}}$, whichever is larger. The mean 


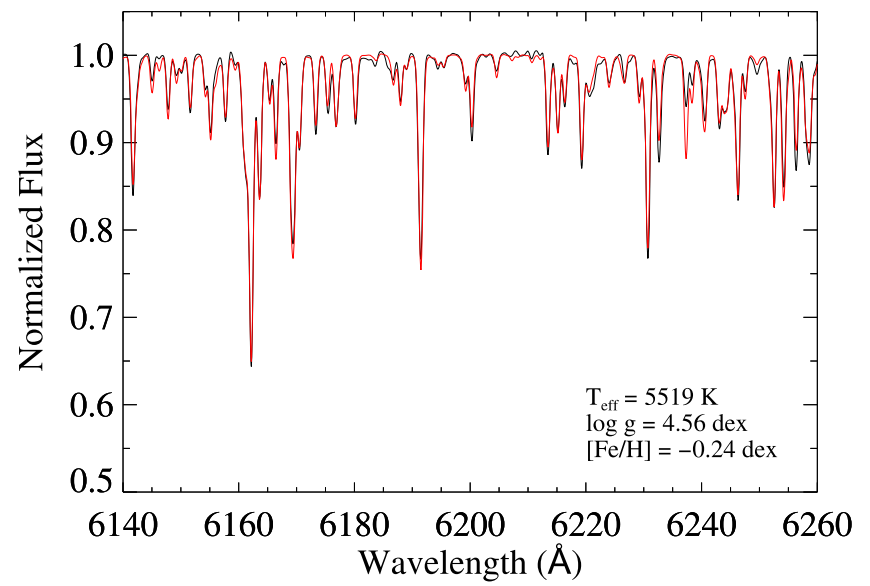

Figure 3. Segment of an observed spectrum of HIP 98677 (gray line). The red line represents the best-fitting synthetic model spectrum in the SMT analysis. The spectral resolution was degraded to $R \sim 10,000$ (see text).

errors are $79 \mathrm{~K}, 0.097 \mathrm{dex}$, and $0.067 \mathrm{dex}$ in $T_{\mathrm{eff}}, \log g$, and $[\mathrm{Fe} / \mathrm{H}]$, respectively.

We repeated the above search for stellar parameters $(\log g$ and $[\mathrm{Fe} / \mathrm{H}]$ ) while holding $T_{\text {eff }}$ fixed using the IRFM relation (Casagrande et al. 2010). We refer to this approach as SMT2, as opposed to SMT3, based on the full three-parameter fitting above. The polynomial equation in the IRFM relation includes metallicity terms, so we derived $T_{\text {eff }}$ using $B-V$ photometry at an initial $[\mathrm{Fe} / \mathrm{H}]$ as derived above. The photometric temperature led to the new $\log g$ and $[\mathrm{Fe} / \mathrm{H}]$ estimates. With these values, we took one more iteration to estimate photometric $T_{\text {eff }}$ and found the best-fitting metallicity and surface gravity for our target stars. The 9th through 14th columns in Table 6 show these values and their errors. The error in $T_{\text {eff }}$ was computed assuming $0.02 \mathrm{mag}$ error in the $B-V$ photometry. The typical errors in $T_{\text {eff }}, \log g$, and $[\mathrm{Fe} / \mathrm{H}]$ from this approach are $60 \mathrm{~K}$, $0.020 \mathrm{dex}$, and $0.016 \mathrm{dex}$, respectively. As summarized in Table 5, SMT2 results are generally consistent with those from SMT3; the differences in the derived parameters are $\Delta T_{\text {eff }}=51 \mathrm{~K}, \Delta \log g=0.09$, and $\Delta[\mathrm{Fe} / \mathrm{H}]=0.03$.

Comparisons of stellar parameters with MOOG are shown in Figures 4 and 5 for SMT3 and SMT2, respectively. In each panel, a solid line represents a zero difference, and a dashed line is an average difference between MOOG and SMT3/ SMT2. Corrected values of $[\mathrm{Fe} / \mathrm{H}]$ from MOOG were used in all comparisons. The SMT3 $T_{\text {eff }}$ estimates are on average higher than those from MOOG, but the difference is small $\left(\Delta T_{\text {eff }}=37\right.$ $\mathrm{K})$. Similarly, the MOOG $T_{\text {eff }}$ estimates are higher than those from SMT2, but the average difference is negligible $(19 \mathrm{~K})$. As discussed above, $\log g$ values from MOOG were probably underestimated, and the comparison with SMT suggests that the difference in $\log g$ is correlated with its metallicity. The metallicities from both SMT3 and SMT2 are systematically higher than those from MOOG by $\Delta[\mathrm{Fe} / \mathrm{H}]=0.09 \mathrm{dex}$ and 0.05 dex, respectively.

Figure 6 displays comparisons in $T_{\text {eff }}, \log g$, and $[\mathrm{Fe} / \mathrm{H}]$ between SMT3 and VF05. The temperatures and metallicities from SMT3 are systematically higher than those from VF05 by $\Delta T_{\text {eff }}=85 \mathrm{~K}$ and $\Delta[\mathrm{Fe} / \mathrm{H}]=0.07 \mathrm{dex}$ (see Table 5). Given the correlation between the two quantities, it seems likely that a higher $T_{\text {eff }}$ has led to a higher $[\mathrm{Fe} / \mathrm{H}]$. There also appears to be a correlation between the $\log g$ difference and metallicity, although the trend is much weaker than those seen in the comparisons of VF05 with MOOG.

The $1 \sigma$ dispersion in the $T_{\text {eff }}$ comparison between SMT3 and VF05 is $95 \mathrm{~K}$. This is consistent with a reported internal precision in each method (44 K and $79 \mathrm{~K}$ for VF05 and SMT3, respectively). Similarly, a $1 \sigma$ dispersion in the $T_{\text {eff }}$ comparison between SMT3 and MOOG is $101 \mathrm{~K}$. This suggests that MOOG temperatures have internal errors of $\sim 90 \mathrm{~K}$, and is consistent with our earlier estimate based on a comparison with VF05. Similarly, a $1 \sigma$ dispersion in the $[\mathrm{Fe} / \mathrm{H}]$ comparison between SMT3 and VF05 is 0.06 dex, which is not far from a quadrature sum of individual internal precision measurements (0.030 dex for VF05 and 0.067 dex for SMT3). A $1 \sigma$ dispersion in the $[\mathrm{Fe} / \mathrm{H}]$ comparison between SMT3 and MOOG is 0.08 dex, and is broadly consistent with $\sigma([\mathrm{Fe} / \mathrm{H}])=0.05-0.08$ for our MOOG results (see Section 3.1).

In terms of a zero point in metallicity, all of the above comparisons suggest that metallicity estimates from SMT3 are about 0.1 dex higher than those from VF05. The level of the systematic offset is not alarmingly large, and an $\sim 0.1$ dex systematic offset in $[\mathrm{Fe} / \mathrm{H}]$ is not uncommon among different spectroscopic analyses (e.g., Torres et al. 2012). A similar offset was also found from a comparison with spectroscopic metallicities in Casagrande et al. (2010, see Table 5): Although there are only 11 stars available in the comparison, the mean difference is $\Delta[\mathrm{Fe} / \mathrm{H}]=0.14 \mathrm{dex}$ in the sense that SMT3 predicts higher metallicities. Given the systematic nature of the difference, we decided to adjust SMT3 metallicities for our sample stars by a constant offset $(\Delta[\mathrm{Fe} / \mathrm{H}]=0.07 \mathrm{dex})$, to be consistent with the metallicity scale of VF05 (and MOOG).

The eighth column in Table 6 (" $[\mathrm{Fe} / \mathrm{H}]_{\text {corr }}$ ") lists metallicities from SMT3 after the zero-point correction. In Figure 7, a black histogram shows a distribution of effective temperature (left panel) and metallicity (right panel) for all of our sample stars as obtained from SMT3. The red shaded histogram represents a subset of these stars, which was analyzed using MOOG. Our sample covers $4800 \lesssim T_{\text {eff }}(\mathrm{K}) \lesssim 5900$ and $-0.8 \lesssim[\mathrm{Fe} / \mathrm{H}] \lesssim 0.2$, and there is no correlation found between $T_{\text {eff }}$ and $[\mathrm{Fe} / \mathrm{H}]$ in either of these two parallel approaches.

\section{RESULTS}

The goal of this work is to identify hypothesized subluminous field stars in the solar neighborhood, which have similar photometric properties with those in the Pleiades. Such stars are sub-luminous in the sense that they would reveal themselves as having fainter absolute magnitudes from Hipparcos than those inferred from MS fitting. If the Hipparcos parallaxes are correct, luminosities of these stars were overestimated in MS fitting for still unknown reasons. Because the luminosity of MS stars is strongly dependent on metallicity, in the following, we first establish a metallicity sensitivity for stellar colors and magnitudes using star samples in the literature that have accurate parallaxes and metallicities (Section 4.1). The comparison in $M_{V}$ between Hipparcos and MS fitting is presented in Section 4.2 for our KPNO sample. We utilize our metallicity measurements from both MOOG and SMT analyses to check our results against potential systematic errors in the adopted metallicity values. Although our sample selection was not designed to find young stars in the solar 
Table 6

Stellar Parameters derived using SMT

\begin{tabular}{|c|c|c|c|c|c|c|c|c|c|c|c|c|c|}
\hline \multirow[b]{2}{*}{ HIP } & \multicolumn{7}{|c|}{ SMT3 } & \multicolumn{6}{|c|}{ SMT2 } \\
\hline & $\begin{array}{l}T_{\text {eff }} \\
(\mathrm{K})\end{array}$ & $\begin{array}{c}\sigma\left(T_{\text {eff }}\right) \\
(\mathrm{K})\end{array}$ & $\log g$ & $\sigma(\log g)$ & {$[\mathrm{Fe} / \mathrm{H}]$} & $\sigma([\mathrm{Fe} / \mathrm{H}])$ & {$[\mathrm{Fe} / \mathrm{H}]_{\text {corr }}{ }^{a}$} & $\begin{array}{l}T_{\text {eff }} \\
(\mathrm{K})\end{array}$ & $\begin{array}{c}\sigma\left(T_{\text {eff }}\right) \\
(\mathrm{K})\end{array}$ & $\log g$ & $\sigma(\log g)$ & {$[\mathrm{Fe} / \mathrm{H}]$} & $\sigma([\mathrm{Fe} / \mathrm{H}])$ \\
\hline 1674 & 5127 & 71 & 4.45 & 0.054 & -0.31 & 0.019 & -0.38 & 5248 & 37 & 4.67 & 0.011 & -0.24 & 0.009 \\
\hline 4907 & 5528 & 24 & 4.67 & 0.128 & -0.01 & 0.070 & -0.09 & 5429 & 57 & 4.54 & 0.016 & -0.07 & 0.013 \\
\hline 5313 & 5865 & 72 & 4.49 & 0.053 & -0.18 & 0.021 & -0.26 & 5839 & 47 & 4.44 & 0.011 & -0.19 & 0.010 \\
\hline 5521 & 5708 & 48 & 4.81 & 0.133 & -0.02 & 0.062 & -0.09 & 5477 & 58 & 4.42 & 0.029 & -0.17 & 0.015 \\
\hline 9172 & 5670 & 54 & 4.58 & 0.089 & +0.01 & 0.072 & -0.06 & 5743 & 69 & 4.61 & 0.015 & +0.05 & 0.014 \\
\hline 9829 & 5634 & 31 & 4.11 & 0.030 & -0.26 & 0.012 & -0.34 & 5687 & 44 & 4.19 & 0.009 & -0.23 & 0.009 \\
\hline 10276 & 5465 & 18 & 4.67 & 0.125 & -0.06 & 0.065 & -0.13 & 5360 & 40 & 4.48 & 0.013 & -0.13 & 0.011 \\
\hline 10629 & 5635 & 26 & 4.34 & 0.033 & -0.42 & 0.023 & -0.49 & 5493 & 43 & 4.12 & 0.011 & -0.54 & 0.011 \\
\hline 11507 & 5662 & 39 & 4.34 & 0.037 & -0.05 & 0.055 & -0.12 & 5720 & 45 & 4.39 & 0.010 & -0.02 & 0.009 \\
\hline 12685 & 5856 & 122 & 4.58 & 0.137 & -0.11 & 0.069 & -0.19 & 5871 & 67 & 4.59 & 0.015 & -0.10 & 0.014 \\
\hline 13771 & 5595 & 19 & 4.24 & 0.021 & -0.23 & 0.016 & -0.31 & 5640 & 43 & 4.31 & 0.009 & -0.20 & 0.007 \\
\hline 14241 & 5509 & 9 & 4.16 & 0.014 & -0.44 & 0.027 & -0.51 & 5577 & 43 & 4.20 & 0.010 & -0.39 & 0.009 \\
\hline 14300 & 5775 & 54 & 4.52 & 0.060 & -0.14 & 0.033 & -0.21 & 5578 & 42 & 4.17 & 0.016 & -0.26 & 0.011 \\
\hline 15062 & 5754 & 61 & 4.24 & 0.032 & -0.32 & 0.016 & -0.39 & 5767 & 65 & 4.27 & 0.023 & -0.31 & 0.014 \\
\hline 15442 & 5803 & 84 & 4.49 & 0.081 & -0.12 & 0.052 & -0.19 & 5711 & 64 & 4.34 & 0.017 & -0.17 & 0.013 \\
\hline 17265 & 5271 & 80 & 4.29 & 0.045 & -0.58 & 0.100 & -0.66 & 5326 & 59 & 4.36 & 0.012 & -0.54 & 0.012 \\
\hline 18324 & 4991 & 150 & 4.30 & 0.059 & -0.42 & 0.044 & -0.49 & 5058 & 50 & 4.39 & 0.015 & -0.37 & 0.013 \\
\hline 20722 & 5742 & 86 & 4.39 & 0.101 & +0.05 & 0.112 & -0.03 & 5799 & 70 & 4.53 & 0.015 & +0.08 & 0.013 \\
\hline 21276 & 5477 & 14 & 4.68 & 0.156 & +0.00 & 0.094 & -0.08 & 5357 & 56 & 4.55 & 0.016 & -0.07 & 0.014 \\
\hline 21571 & 5632 & 31 & 4.41 & 0.044 & -0.16 & 0.033 & -0.23 & 5569 & 42 & 4.31 & 0.009 & -0.21 & 0.009 \\
\hline 21832 & 5757 & 84 & 4.32 & 0.075 & -0.47 & 0.051 & -0.54 & 5740 & 67 & 4.31 & 0.012 & -0.47 & 0.010 \\
\hline 22175 & 5661 & 53 & 4.43 & 0.075 & -0.08 & 0.067 & -0.15 & 5650 & 63 & 4.41 & 0.014 & -0.08 & 0.014 \\
\hline 23431 & 5450 & 24 & 4.40 & 0.069 & -0.43 & 0.048 & -0.51 & 5227 & 59 & 4.11 & 0.016 & -0.60 & 0.017 \\
\hline 23786 & 5423 & 24 & 4.71 & 0.105 & -0.04 & 0.052 & -0.11 & 5269 & 56 & 4.49 & 0.017 & -0.13 & 0.015 \\
\hline 26505 & 5054 & 73 & 4.39 & 0.033 & -0.42 & 0.026 & -0.50 & 5117 & 36 & 4.48 & 0.011 & -0.38 & 0.010 \\
\hline 30862 & 5676 & 37 & 4.13 & 0.034 & -0.48 & 0.040 & -0.55 & 5690 & 46 & 4.15 & 0.010 & -0.48 & 0.010 \\
\hline 44259 & 5210 & 52 & 4.69 & 0.072 & -0.29 & 0.018 & -0.36 & 5147 & 36 & 4.59 & 0.010 & -0.32 & 0.010 \\
\hline 44262 & 5743 & 58 & 4.41 & 0.047 & +0.03 & 0.075 & -0.05 & 5791 & 51 & 4.48 & 0.010 & +0.05 & 0.008 \\
\hline 52278 & 5671 & 45 & 4.18 & 0.029 & -0.41 & 0.032 & -0.48 & 5505 & 62 & 4.00 & 0.024 & -0.54 & 0.016 \\
\hline 52470 & 5397 & 32 & 4.44 & 0.068 & -0.38 & 0.027 & -0.45 & 5242 & 55 & 4.20 & 0.016 & -0.49 & 0.014 \\
\hline 54906 & 5325 & 63 & 4.63 & 0.144 & -0.15 & 0.054 & -0.22 & 5133 & 51 & 4.37 & 0.015 & -0.29 & 0.016 \\
\hline 55210 & 5552 & 15 & 4.58 & 0.070 & -0.10 & 0.035 & -0.17 & 5401 & 40 & 4.34 & 0.012 & -0.21 & 0.011 \\
\hline 56092 & 5096 & 104 & 4.69 & 0.110 & -0.19 & 0.044 & -0.27 & 4850 & 32 & 4.31 & 0.014 & -0.36 & 0.012 \\
\hline 56337 & 5560 & 20 & 4.60 & 0.072 & -0.05 & 0.046 & -0.12 & 5488 & 43 & 4.41 & 0.016 & -0.09 & 0.010 \\
\hline 56837 & 5569 & 21 & 4.37 & 0.044 & -0.29 & 0.014 & -0.36 & 5571 & 60 & 4.38 & 0.016 & -0.28 & 0.015 \\
\hline 57939 & 5147 & 69 & 4.47 & 0.067 & -1.31 & 0.195 & -1.39 & 5141 & 55 & 4.46 & 0.010 & -1.32 & 0.010 \\
\hline 57992 & 5496 & 13 & 4.47 & 0.059 & -0.30 & 0.013 & -0.37 & 5380 & 57 & 4.30 & 0.016 & -0.38 & 0.015 \\
\hline 58949 & 5332 & 54 & 4.37 & 0.072 & -0.23 & 0.029 & -0.30 & 5444 & 57 & 4.55 & 0.016 & -0.15 & 0.015 \\
\hline 60074 & 5842 & 68 & 4.59 & 0.079 & -0.03 & 0.054 & -0.10 & 5869 & 49 & 4.62 & 0.010 & -0.02 & 0.010 \\
\hline 60268 & 5607 & 24 & 4.16 & 0.017 & -0.67 & 0.069 & -0.74 & 5724 & 69 & 4.34 & 0.015 & -0.59 & 0.014 \\
\hline 63322 & 5156 & 88 & 4.63 & 0.094 & -0.03 & 0.060 & -0.10 & 5142 & 38 & 4.63 & 0.010 & -0.04 & 0.010 \\
\hline 63346 & 5589 & 36 & 4.29 & 0.035 & -0.42 & 0.048 & -0.49 & 5484 & 61 & 4.12 & 0.015 & -0.50 & 0.015 \\
\hline 63636 & 5752 & 90 & 4.53 & 0.119 & -0.03 & 0.091 & -0.11 & 5725 & 64 & 4.46 & 0.015 & -0.04 & 0.015 \\
\hline 64076 & 5802 & 64 & 4.38 & 0.043 & -0.16 & 0.031 & -0.23 & 5843 & 46 & 4.46 & 0.011 & -0.13 & 0.010 \\
\hline 64131 & 5625 & 28 & 3.95 & 0.044 & -0.65 & 0.079 & -0.72 & 5705 & 48 & 4.09 & 0.010 & -0.58 & 0.010 \\
\hline 65165 & 5489 & 21 & 4.61 & 0.117 & +0.00 & 0.086 & -0.07 & 5388 & 58 & 4.41 & 0.018 & -0.05 & 0.015 \\
\hline 65352 & 5344 & 30 & 4.57 & 0.065 & -0.27 & 0.013 & -0.34 & 5179 & 37 & 4.29 & 0.016 & -0.38 & 0.010 \\
\hline 65355 & 5171 & 94 & 4.59 & 0.103 & -0.26 & 0.033 & -0.33 & 5026 & 49 & 4.34 & 0.018 & -0.36 & 0.015 \\
\hline 65935 & 5123 & 99 & 4.69 & 0.104 & -0.01 & 0.068 & -0.08 & 5058 & 36 & 4.59 & 0.010 & -0.04 & 0.010 \\
\hline 66509 & 5484 & 17 & 4.15 & 0.025 & -0.63 & 0.094 & -0.70 & 5595 & 65 & 4.35 & 0.014 & -0.54 & 0.014 \\
\hline 67211 & 4817 & 155 & 4.40 & 0.090 & -0.30 & 0.045 & -0.37 & 4867 & 32 & 4.49 & 0.010 & -0.27 & 0.010 \\
\hline 67282 & 5432 & 18 & 4.83 & 0.089 & +0.00 & 0.043 & -0.07 & 5217 & 38 & 4.43 & 0.016 & -0.11 & 0.011 \\
\hline 67773 & 5336 & 43 & 4.65 & 0.095 & -0.20 & 0.030 & -0.27 & 5203 & 52 & 4.44 & 0.016 & -0.30 & 0.015 \\
\hline 67904 & 5268 & 53 & 4.43 & 0.035 & -0.29 & 0.022 & -0.37 & 5566 & 35 & 4.22 & 0.078 & -0.08 & 0.013 \\
\hline 70253 & 5645 & 41 & 4.16 & 0.022 & -0.54 & 0.066 & -0.61 & 5745 & 66 & 4.33 & 0.016 & -0.49 & 0.015 \\
\hline 70319 & 5684 & 30 & 4.21 & 0.020 & -0.32 & 0.009 & -0.40 & 5729 & 37 & 4.26 & 0.007 & -0.30 & 0.007 \\
\hline 71720 & 5316 & 45 & 4.61 & 0.087 & -0.06 & 0.052 & -0.13 & 5121 & 51 & 4.31 & 0.022 & -0.19 & 0.015 \\
\hline 72577 & 4965 & 107 & 4.67 & 0.090 & -0.07 & 0.053 & -0.15 & 4916 & 35 & 4.61 & 0.010 & -0.11 & 0.010 \\
\hline 72663 & 5591 & 37 & 4.31 & 0.046 & -0.22 & 0.034 & -0.29 & 5613 & 61 & 4.35 & 0.014 & -0.20 & 0.014 \\
\hline 72703 & 5682 & 51 & 4.68 & 0.128 & -0.06 & 0.064 & -0.13 & 5561 & 61 & 4.52 & 0.015 & -0.14 & 0.014 \\
\hline 73005 & 5236 & 73 & 4.39 & 0.055 & -0.33 & 0.019 & -0.40 & 5183 & 52 & 4.32 & 0.015 & -0.36 & 0.013 \\
\hline 73138 & 5382 & 45 & 4.64 & 0.162 & +0.02 & 0.118 & -0.05 & 5403 & 62 & 4.65 & 0.014 & +0.04 & 0.014 \\
\hline
\end{tabular}


Table 6

(Continued)

\begin{tabular}{|c|c|c|c|c|c|c|c|c|c|c|c|c|c|}
\hline \multirow[b]{2}{*}{ HIP } & \multicolumn{7}{|c|}{ SMT3 } & \multicolumn{6}{|c|}{ SMT2 } \\
\hline & $\begin{array}{l}T_{\text {eff }} \\
(\mathrm{K})\end{array}$ & $\begin{array}{c}\sigma\left(T_{\text {eff }}\right) \\
(\mathrm{K})\end{array}$ & $\log g$ & $\sigma(\log g)$ & {$[\mathrm{Fe} / \mathrm{H}]$} & $\sigma([\mathrm{Fe} / \mathrm{H}])$ & {$[\mathrm{Fe} / \mathrm{H}]_{\text {corr }}{ }^{\mathrm{a}}$} & $\begin{array}{l}T_{\text {eff }} \\
(\mathrm{K})\end{array}$ & $\begin{array}{c}\sigma\left(T_{\text {eff }}\right) \\
(\mathrm{K})\end{array}$ & $\log g$ & $\sigma(\log g)$ & {$[\mathrm{Fe} / \mathrm{H}]$} & $\sigma([\mathrm{Fe} / \mathrm{H}])$ \\
\hline 73677 & 5673 & 44 & 4.12 & 0.028 & -0.51 & 0.054 & -0.59 & 5686 & 66 & 4.15 & 0.012 & -0.51 & 0.012 \\
\hline 73845 & 6456 & 249 & 4.46 & 0.089 & +0.18 & 0.126 & +0.10 & 5747 & 64 & 3.62 & 0.082 & -0.39 & 0.036 \\
\hline 74126 & 5689 & 57 & 4.59 & 0.110 & -0.05 & 0.069 & -0.12 & 5717 & 64 & 4.62 & 0.014 & -0.04 & 0.014 \\
\hline 74396 & 5269 & 97 & 4.81 & 0.209 & +0.00 & 0.101 & -0.07 & 5119 & 52 & 4.59 & 0.015 & -0.09 & 0.015 \\
\hline 75059 & 5754 & 64 & 4.34 & 0.041 & -0.11 & 0.049 & -0.18 & 5654 & 62 & 4.23 & 0.015 & -0.16 & 0.014 \\
\hline 75370 & 5485 & 15 & 4.19 & 0.024 & -0.38 & 0.025 & -0.45 & 5617 & 61 & 4.41 & 0.013 & -0.28 & 0.015 \\
\hline 75446 & 5561 & 27 & 4.53 & 0.085 & -0.04 & 0.068 & -0.11 & 5489 & 60 & 4.37 & 0.013 & -0.08 & 0.013 \\
\hline 75703 & 5582 & 34 & 4.81 & 0.168 & -0.13 & 0.052 & -0.20 & 5398 & 56 & 4.54 & 0.020 & -0.26 & 0.015 \\
\hline 76058 & 5818 & 50 & 4.55 & 0.047 & -0.15 & 0.026 & -0.23 & 5729 & 45 & 4.45 & 0.012 & -0.21 & 0.011 \\
\hline 76130 & 5863 & 87 & 4.36 & 0.045 & -0.09 & 0.052 & -0.16 & 5861 & 67 & 4.36 & 0.016 & -0.10 & 0.015 \\
\hline 76330 & 5237 & 69 & 4.81 & 0.140 & +0.02 & 0.074 & -0.06 & 5168 & 53 & 4.67 & 0.015 & -0.02 & 0.015 \\
\hline 76674 & 5476 & 16 & 4.79 & 0.146 & +0.04 & 0.087 & -0.03 & 5483 & 59 & 4.81 & 0.015 & +0.04 & 0.015 \\
\hline 77810 & 5860 & 108 & 4.57 & 0.108 & +0.00 & 0.090 & -0.07 & 5849 & 70 & 4.54 & 0.014 & +0.00 & 0.013 \\
\hline 78028 & 5791 & 100 & 4.31 & 0.051 & -0.06 & 0.084 & -0.13 & 5808 & 68 & 4.32 & 0.015 & -0.05 & 0.015 \\
\hline 78241 & 5393 & 44 & 4.49 & 0.106 & -0.31 & 0.018 & -0.39 & 5315 & 55 & 4.40 & 0.015 & -0.38 & 0.015 \\
\hline 78336 & 5131 & 136 & 4.83 & 0.212 & +0.17 & 0.149 & +0.09 & 5168 & 75 & 4.84 & 0.015 & +0.19 & 0.015 \\
\hline 78550 & 5612 & 37 & 4.40 & 0.067 & -0.27 & 0.019 & -0.34 & 5608 & 61 & 4.41 & 0.011 & -0.28 & 0.011 \\
\hline 78775 & 5336 & 61 & 4.39 & 0.069 & -0.61 & 0.112 & -0.68 & 5311 & 64 & 4.36 & 0.014 & -0.62 & 0.014 \\
\hline 78923 & 5685 & 70 & 4.35 & 0.067 & -0.09 & 0.074 & -0.17 & 5600 & 61 & 4.24 & 0.013 & -0.14 & 0.011 \\
\hline 79629 & 5645 & 30 & 4.34 & 0.031 & -0.27 & 0.011 & -0.34 & 5685 & 44 & 4.38 & 0.011 & -0.24 & 0.011 \\
\hline 80243 & 5353 & 40 & 4.65 & 0.140 & +0.11 & 0.108 & +0.04 & 5382 & 60 & 4.69 & 0.016 & +0.13 & 0.013 \\
\hline 80262 & 5624 & 29 & 4.60 & 0.084 & -0.02 & 0.060 & -0.10 & 5524 & 62 & 4.41 & 0.014 & -0.09 & 0.014 \\
\hline 81186 & 5199 & 56 & 4.76 & 0.094 & -0.17 & 0.032 & -0.24 & 5043 & 35 & 4.53 & 0.011 & -0.27 & 0.011 \\
\hline 81288 & 4929 & 156 & 4.44 & 0.085 & -0.83 & 0.146 & -0.91 & 4892 & 64 & 4.38 & 0.014 & -0.86 & 0.013 \\
\hline 81605 & 5602 & 32 & 4.58 & 0.095 & -0.02 & 0.072 & -0.09 & 5540 & 61 & 4.41 & 0.013 & -0.06 & 0.013 \\
\hline 81831 & 5635 & 44 & 4.60 & 0.125 & -0.17 & 0.045 & -0.24 & 5423 & 57 & 4.26 & 0.019 & -0.33 & 0.016 \\
\hline 82120 & 5564 & 16 & 4.29 & 0.023 & -0.35 & 0.015 & -0.42 & 5577 & 43 & 4.30 & 0.009 & -0.34 & 0.009 \\
\hline 82210 & 5807 & 111 & 4.60 & 0.146 & -0.06 & 0.086 & -0.14 & 5665 & 62 & 4.39 & 0.016 & -0.13 & 0.014 \\
\hline 82388 & 5693 & 159 & 4.08 & 0.107 & -0.19 & 0.064 & -0.26 & 6019 & 63 & 4.36 & 0.086 & +0.38 & 0.019 \\
\hline 82644 & 5828 & 105 & 4.59 & 0.126 & -0.08 & 0.073 & -0.15 & 5758 & 65 & 4.45 & 0.015 & -0.12 & 0.015 \\
\hline 82712 & 5595 & 25 & 4.47 & 0.064 & -0.27 & 0.016 & -0.34 & 5491 & 59 & 4.32 & 0.022 & -0.35 & 0.015 \\
\hline 83141 & 4884 & 144 & 4.61 & 0.110 & -0.20 & 0.050 & -0.27 & 4869 & 32 & 4.58 & 0.010 & -0.21 & 0.010 \\
\hline 83276 & 5820 & 52 & 4.00 & 0.044 & -0.11 & 0.032 & -0.18 & 5833 & 47 & 4.10 & 0.011 & -0.10 & 0.011 \\
\hline 83500 & 5852 & 88 & 4.17 & 0.027 & -0.50 & 0.050 & -0.58 & 5705 & 69 & 3.93 & 0.014 & -0.60 & 0.014 \\
\hline 84520 & 5820 & 80 & 4.39 & 0.063 & -0.09 & 0.053 & -0.16 & 5730 & 63 & 4.27 & 0.016 & -0.14 & 0.014 \\
\hline 85235 & 5382 & 31 & 4.44 & 0.060 & -0.36 & 0.018 & -0.43 & 5321 & 56 & 4.35 & 0.015 & -0.39 & 0.015 \\
\hline 85653 & 5439 & 22 & 4.37 & 0.052 & -0.32 & 0.013 & -0.39 & 5387 & 57 & 4.29 & 0.016 & -0.35 & 0.015 \\
\hline 86568 & 5315 & 47 & 4.37 & 0.042 & -0.33 & 0.013 & -0.40 & 5395 & 40 & 4.49 & 0.012 & -0.28 & 0.010 \\
\hline 86765 & 5622 & 35 & 4.34 & 0.040 & -0.19 & 0.031 & -0.26 & 5642 & 62 & 4.37 & 0.014 & -0.17 & 0.014 \\
\hline 87089 & 5184 & 103 & 4.46 & 0.084 & -0.28 & 0.025 & -0.35 & 5258 & 53 & 4.56 & 0.016 & -0.23 & 0.015 \\
\hline 88166 & 5397 & 18 & 4.22 & 0.027 & -0.74 & 0.064 & -0.81 & 5416 & 46 & 4.25 & 0.010 & -0.73 & 0.010 \\
\hline 88972 & 5075 & 129 & 4.50 & 0.100 & -0.12 & 0.060 & -0.19 & 5112 & 51 & 4.56 & 0.015 & -0.09 & 0.015 \\
\hline 90355 & 5620 & 32 & 4.40 & 0.049 & -0.11 & 0.046 & -0.18 & 5580 & 60 & 4.34 & 0.014 & -0.14 & 0.014 \\
\hline 90415 & 4950 & 95 & 4.36 & 0.049 & -0.45 & 0.030 & -0.52 & 4906 & 28 & 4.25 & 0.007 & -0.47 & 0.007 \\
\hline 90708 & 5760 & 84 & 4.39 & 0.073 & -0.06 & 0.079 & -0.13 & 5751 & 65 & 4.38 & 0.015 & -0.06 & 0.015 \\
\hline 91364 & 5396 & 61 & 4.81 & 0.187 & -0.12 & 0.063 & -0.19 & 5127 & 51 & 4.36 & 0.024 & -0.30 & 0.016 \\
\hline 91381 & 5534 & 15 & 4.42 & 0.065 & -0.34 & 0.016 & -0.41 & 5456 & 58 & 4.30 & 0.016 & -0.38 & 0.012 \\
\hline 91438 & 5712 & 41 & 4.54 & 0.065 & -0.13 & 0.035 & -0.20 & 5681 & 62 & 4.45 & 0.030 & -0.14 & 0.012 \\
\hline 91605 & 5071 & 150 & 4.72 & 0.157 & -0.20 & 0.064 & -0.28 & 4925 & 47 & 4.45 & 0.028 & -0.29 & 0.015 \\
\hline 91614 & 5622 & 51 & 4.27 & 0.036 & -0.27 & 0.017 & -0.34 & 5540 & 60 & 4.13 & 0.014 & -0.33 & 0.015 \\
\hline 91905 & 5472 & 11 & 4.44 & 0.052 & -0.22 & 0.018 & -0.30 & 5368 & 40 & 4.32 & 0.012 & -0.30 & 0.011 \\
\hline 92388 & 5449 & 22 & 4.38 & 0.068 & -0.40 & 0.039 & -0.47 & 5426 & 58 & 4.37 & 0.013 & -0.42 & 0.013 \\
\hline 92569 & 5714 & 47 & 4.55 & 0.075 & -0.12 & 0.040 & -0.20 & 5595 & 60 & 4.34 & 0.016 & -0.20 & 0.015 \\
\hline 93195 & 5342 & 44 & 4.69 & 0.131 & +0.04 & 0.087 & -0.03 & 5273 & 56 & 4.62 & 0.014 & +0.00 & 0.014 \\
\hline 93731 & 5414 & 31 & 4.65 & 0.154 & -0.04 & 0.085 & -0.11 & 5315 & 56 & 4.52 & 0.017 & -0.10 & 0.015 \\
\hline 94582 & 5523 & 14 & 4.69 & 0.080 & -0.07 & 0.039 & -0.14 & 5310 & 39 & 4.37 & 0.012 & -0.20 & 0.011 \\
\hline 95727 & 5272 & 66 & 4.16 & 0.061 & -0.30 & 0.162 & -0.37 & 5288 & 38 & 3.47 & 0.256 & -0.37 & 0.196 \\
\hline 96100 & 5437 & 32 & 4.67 & 0.123 & -0.07 & 0.061 & -0.15 & 5276 & 54 & 4.39 & 0.016 & -0.17 & 0.015 \\
\hline 96344 & 5714 & 45 & 4.15 & 0.018 & -0.52 & 0.047 & -0.60 & 5546 & 47 & 3.85 & 0.015 & -0.65 & 0.012 \\
\hline 96634 & 5283 & 51 & 4.54 & 0.072 & -0.10 & 0.043 & -0.18 & 5329 & 40 & 4.60 & 0.010 & -0.07 & 0.010 \\
\hline 96735 & 5179 & 69 & 4.80 & 0.111 & -0.05 & 0.048 & -0.13 & 5122 & 36 & 4.66 & 0.011 & -0.07 & 0.011 \\
\hline 97640 & 5703 & 70 & 4.68 & 0.223 & -1.26 & 0.224 & -1.33 & 4996 & 79 & 3.99 & 0.055 & -1.89 & 0.087 \\
\hline
\end{tabular}


Table 6

(Continued)

\begin{tabular}{|c|c|c|c|c|c|c|c|c|c|c|c|c|c|}
\hline \multirow[b]{2}{*}{ HIP } & \multicolumn{7}{|c|}{ SMT3 } & \multicolumn{6}{|c|}{ SMT2 } \\
\hline & $\begin{array}{l}T_{\text {eff }} \\
(\mathrm{K})\end{array}$ & $\begin{array}{c}\sigma\left(T_{\text {eff }}\right) \\
(\mathrm{K})\end{array}$ & $\log g$ & $\sigma(\log g)$ & {$[\mathrm{Fe} / \mathrm{H}]$} & $\sigma([\mathrm{Fe} / \mathrm{H}])$ & {$[\mathrm{Fe} / \mathrm{H}]_{\text {corr }}{ }^{\mathrm{a}}$} & $\begin{array}{l}T_{\text {eff }} \\
(\mathrm{K})\end{array}$ & $\begin{array}{c}\sigma\left(T_{\text {eff }}\right) \\
(\mathrm{K})\end{array}$ & $\log g$ & $\sigma(\log g)$ & {$[\mathrm{Fe} / \mathrm{H}]$} & $\sigma([\mathrm{Fe} / \mathrm{H}])$ \\
\hline 97668 & 5056 & 74 & 4.59 & 0.065 & -0.11 & 0.035 & -0.19 & 5023 & 35 & 4.58 & 0.010 & -0.14 & 0.010 \\
\hline 98288 & 5778 & 63 & 4.24 & 0.026 & -0.27 & 0.011 & -0.34 & 5780 & 46 & 4.23 & 0.011 & -0.27 & 0.010 \\
\hline 98413 & 5102 & 69 & 4.73 & 0.077 & -0.12 & 0.035 & -0.20 & 5027 & 35 & 4.60 & 0.013 & -0.17 & 0.011 \\
\hline 98559 & 5595 & 23 & 4.34 & 0.037 & -0.08 & 0.046 & -0.15 & 5575 & 43 & 4.32 & 0.008 & -0.09 & 0.008 \\
\hline 98677 & 5519 & 15 & 4.56 & 0.090 & -0.24 & 0.021 & -0.31 & 5389 & 56 & 4.34 & 0.015 & -0.33 & 0.015 \\
\hline 98792 & 5045 & 73 & 4.38 & 0.036 & -0.57 & 0.045 & -0.64 & 5062 & 32 & 4.40 & 0.008 & -0.56 & 0.008 \\
\hline 98965 & 5651 & 46 & 4.63 & 0.123 & -0.11 & 0.053 & -0.19 & 5474 & 58 & 4.34 & 0.017 & -0.25 & 0.016 \\
\hline 99355 & 5135 & 94 & 4.68 & 0.112 & -0.16 & 0.047 & -0.24 & 5035 & 49 & 4.55 & 0.014 & -0.23 & 0.015 \\
\hline 99774 & 5583 & 18 & 4.04 & 0.033 & -0.77 & 0.075 & -0.85 & 5727 & 51 & 4.24 & 0.014 & -0.68 & 0.010 \\
\hline 99965 & 5512 & 28 & 4.46 & 0.083 & +0.09 & 0.102 & +0.02 & 5455 & 65 & 4.38 & 0.015 & +0.06 & 0.015 \\
\hline 100259 & 5928 & 110 & 4.66 & 0.124 & -0.07 & 0.063 & -0.14 & 5793 & 65 & 4.40 & 0.016 & -0.16 & 0.015 \\
\hline 101579 & 4941 & 110 & 4.63 & 0.083 & -0.04 & 0.054 & -0.11 & 4936 & 37 & 4.63 & 0.009 & -0.04 & 0.009 \\
\hline 101997 & 5527 & 15 & 4.40 & 0.059 & -0.21 & 0.030 & -0.28 & 5473 & 58 & 4.35 & 0.014 & -0.24 & 0.014 \\
\hline 102290 & 5859 & 62 & 4.48 & 0.053 & -0.03 & 0.048 & -0.10 & 5899 & 50 & 4.45 & 0.010 & +0.00 & 0.010 \\
\hline 102521 & 5924 & 118 & 4.56 & 0.100 & -0.05 & 0.070 & -0.13 & 5833 & 66 & 4.41 & 0.020 & -0.12 & 0.014 \\
\hline 103611 & 5678 & 35 & 4.60 & 0.073 & -0.07 & 0.043 & -0.14 & 5646 & 45 & 4.59 & 0.009 & -0.09 & 0.009 \\
\hline 103895 & 5453 & 13 & 4.20 & 0.012 & -0.45 & 0.038 & -0.52 & 5519 & 42 & 4.34 & 0.013 & -0.41 & 0.010 \\
\hline 103896 & 5839 & 66 & 4.51 & 0.060 & -0.08 & 0.043 & -0.16 & 5772 & 45 & 4.39 & 0.011 & -0.13 & 0.010 \\
\hline 104375 & 5302 & 64 & 4.75 & 0.159 & -0.23 & 0.025 & -0.31 & 5042 & 50 & 4.28 & 0.034 & -0.39 & 0.016 \\
\hline 104733 & 5790 & 49 & 4.52 & 0.054 & -0.13 & 0.030 & -0.20 & 5709 & 45 & 4.43 & 0.010 & -0.17 & 0.007 \\
\hline 106231 & 4897 & 108 & 4.55 & 0.169 & -0.99 & 0.113 & -1.06 & 4546 & 46 & 4.39 & 0.013 & -1.26 & 0.015 \\
\hline 106949 & 5592 & 17 & 4.25 & 0.012 & -0.46 & 0.028 & -0.53 & 5628 & 45 & 4.28 & 0.010 & -0.43 & 0.010 \\
\hline 107038 & 5230 & 66 & 4.65 & 0.110 & +0.01 & 0.071 & -0.07 & 5143 & 54 & 4.56 & 0.015 & -0.04 & 0.014 \\
\hline 107700 & 5217 & 97 & 4.60 & 0.130 & -0.13 & 0.058 & -0.20 & 5100 & 50 & 4.40 & 0.016 & -0.20 & 0.015 \\
\hline 108774 & 5708 & 61 & 4.65 & 0.128 & -0.07 & 0.066 & -0.15 & 5561 & 60 & 4.38 & 0.015 & -0.15 & 0.015 \\
\hline 108947 & 5388 & 49 & 4.60 & 0.161 & -0.03 & 0.104 & -0.10 & 5315 & 57 & 4.54 & 0.016 & -0.08 & 0.016 \\
\hline 109310 & 5883 & 60 & 4.39 & 0.034 & -0.09 & 0.034 & -0.16 & 5827 & 46 & 4.35 & 0.011 & -0.12 & 0.010 \\
\hline 110508 & 5662 & 41 & 4.57 & 0.090 & -0.09 & 0.050 & -0.16 & 5542 & 60 & 4.33 & 0.017 & -0.17 & 0.015 \\
\hline 111883 & 5132 & 86 & 4.65 & 0.099 & -0.20 & 0.033 & -0.27 & 5019 & 34 & 4.45 & 0.020 & -0.26 & 0.010 \\
\hline 111888 & 5029 & 121 & 4.78 & 0.127 & -0.15 & 0.058 & -0.22 & 5021 & 49 & 4.77 & 0.015 & -0.15 & 0.015 \\
\hline 111977 & 5735 & 58 & 4.34 & 0.051 & -0.43 & 0.035 & -0.50 & 5687 & 65 & 4.29 & 0.015 & -0.45 & 0.015 \\
\hline 112245 & 5565 & 19 & 4.40 & 0.047 & -0.12 & 0.041 & -0.20 & 5454 & 57 & 4.29 & 0.015 & -0.21 & 0.013 \\
\hline 112870 & 4944 & 191 & 4.34 & 0.072 & -0.63 & 0.118 & -0.71 & 4974 & 57 & 4.39 & 0.015 & -0.62 & 0.015 \\
\hline 113231 & 5670 & 52 & 4.24 & 0.022 & -0.32 & 0.014 & -0.39 & 5675 & 63 & 4.27 & 0.014 & -0.32 & 0.013 \\
\hline 113884 & 5815 & 52 & 4.24 & 0.014 & -0.25 & 0.013 & -0.32 & 5785 & 38 & 4.17 & 0.008 & -0.26 & 0.008 \\
\hline 113989 & 5575 & 33 & 4.11 & 0.040 & -0.46 & 0.059 & -0.53 & 5669 & 64 & 4.24 & 0.013 & -0.39 & 0.014 \\
\hline 114340 & 5667 & 31 & 4.59 & 0.061 & +0.01 & 0.047 & -0.07 & 5705 & 47 & 4.65 & 0.010 & +0.02 & 0.010 \\
\hline 114385 & 5794 & 71 & 4.55 & 0.081 & +0.06 & 0.086 & -0.01 & 5771 & 65 & 4.41 & 0.013 & +0.05 & 0.013 \\
\hline 115194 & 5040 & 89 & 4.37 & 0.035 & -0.61 & 0.061 & -0.69 & 5218 & 40 & 4.62 & 0.011 & -0.48 & 0.011 \\
\hline 115411 & 5502 & 14 & 4.30 & 0.038 & -0.33 & 0.015 & -0.40 & 5623 & 61 & 4.54 & 0.021 & -0.24 & 0.015 \\
\hline 115445 & 5180 & 167 & 4.76 & 0.225 & -0.10 & 0.086 & -0.17 & 5004 & 48 & 4.42 & 0.046 & -0.20 & 0.014 \\
\hline 116005 & 5129 & 69 & 4.72 & 0.075 & -0.02 & 0.046 & -0.09 & 5117 & 31 & 4.69 & 0.009 & -0.03 & 0.007 \\
\hline 116410 & 5484 & 13 & 4.34 & 0.041 & -0.31 & 0.013 & -0.38 & 5399 & 57 & 4.21 & 0.016 & -0.36 & 0.015 \\
\hline 118207 & 5691 & 37 & 4.25 & 0.018 & -0.27 & 0.012 & -0.34 & 5697 & 45 & 4.23 & 0.009 & -0.26 & 0.008 \\
\hline 118251 & 5696 & 69 & 4.20 & 0.016 & -0.40 & 0.039 & -0.47 & 5677 & 64 & 4.18 & 0.014 & -0.41 & 0.014 \\
\hline 118278 & 5533 & 15 & 4.41 & 0.052 & -0.07 & 0.057 & -0.15 & 5460 & 59 & 4.27 & 0.012 & -0.11 & 0.012 \\
\hline
\end{tabular}

Note.

${ }^{\text {a }}$ Corrected to match the metallicity scale of VF05 (see text).

neighborhood, a small number of stars were found in our sample with strong lithium absorptions and/or chromospheric activity levels that are characteristic of stars in the Pleiades. In Section 4.3 we use these stars to test a hypothesis that the Pleiades' distance is overestimated in MS fitting due to the young ages of its members. Finally, we present our best estimate of an MS-fitting distance to the Pleiades based on the observed MS of the Hyades and the empirical metallicity sensitivities of stellar colors and magnitudes (Section 4.4).

\subsection{Metallicity Sensitivity Function}

An absolute magnitude of an ith star in our sample can be computed either based on the Hipparcos parallax $\left(M_{V, i}^{\mathrm{HIP}}\right)$ or MS fitting $\left(M_{V, i}^{\mathrm{MS}}\right)$. The difference between the two $M_{V}$ estimates is equal to a difference in distance modulus:

$$
\begin{gathered}
\Delta M_{V, i} \equiv M_{V, i}^{\mathrm{HIP}}-M_{V, i}^{\mathrm{MS}} \\
=\left(V-M_{V}\right)_{0, i}^{\mathrm{MS}}-\left(V-M_{V}\right)_{0, i}^{\mathrm{HIP}} .
\end{gathered}
$$



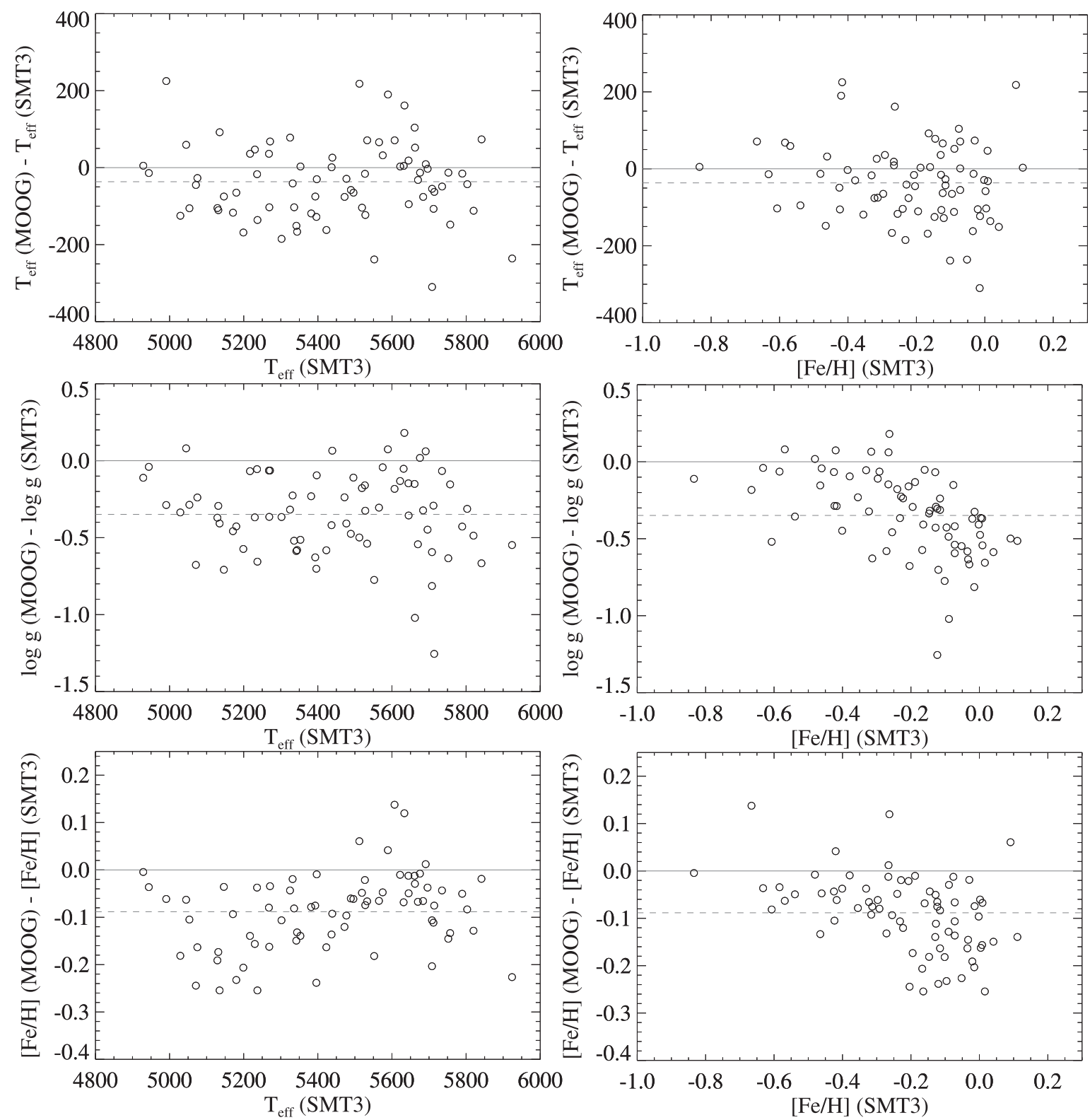

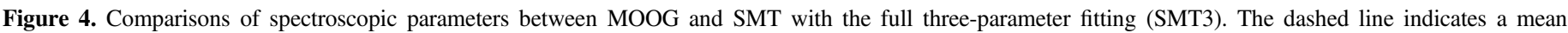

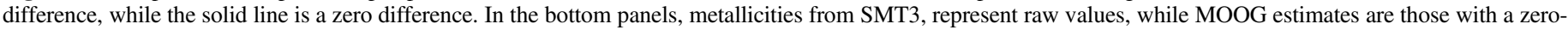
point adjustment (see text).

While $M_{V, i}^{\mathrm{HIP}}$ can be directly estimated from a $V$-band magnitude and a trigonometric parallax for individual stars, the MS-fitting approach requires a well-defined set of color-magnitude relations over a wide range of metallicity. More specifically, $M_{V, i}^{\mathrm{MS}}$ in the above equations is a function of both temperature (or color) and metallicity, and should be known a priori to derive a distance from MS fitting. Within the temperature range of our sample, metal-poor MS stars are fainter than metal-rich stars at a given $B-V$ color, and the amount of offset in $M_{V}$ as a function of metallicity can be constrained either from observations or theory.

In this paper, we employed a purely empirical approach, instead of relying on theoretical stellar isochrones, to search for anomalously sub-luminous stars in the solar neighborhood. We utilized an observed MS of the Hyades at its well-known metallicity and distance (Pinsonneault et al. 2004), and applied metallicity effects on stellar colors and magnitudes. This was done by rewriting the absolute magnitude of a star as

$$
M_{V, i}^{\mathrm{MS}}=M_{V}^{\text {Hyades }}\left(X_{i}\right)+\overline{\delta M_{V}}\left([\mathrm{Fe} / \mathrm{H}]_{i}\right),
$$

where $M_{V}^{\text {Hyades }}\left(X_{i}\right)$ is the $M_{V}$ of the Hyades' MS at a given color (or temperature) of a star in $X$ passband, such as in $B-V$. The $\overline{\delta M_{V}}\left([\mathrm{Fe} / \mathrm{H}]_{i}\right)$ represents a metallicity term that may also depend on colors. However, the color range of our KPNO sample is sufficiently narrow that color-magnitude relations at different metallicities are almost parallel to one another (see the theoretical lines in the top lefthand panel of Figure 1), ${ }^{15}$ and

\footnotetext{
${ }^{15}$ The approximate behavior of stars with differing metallicities can be understood with a simple homology relation (e.g., Portinari et al. 2010; see their Equation (4)). Note, however, that the influence of helium enrichment should be disentangled in order to measure the metallicity effect on stellar luminosity.
} 

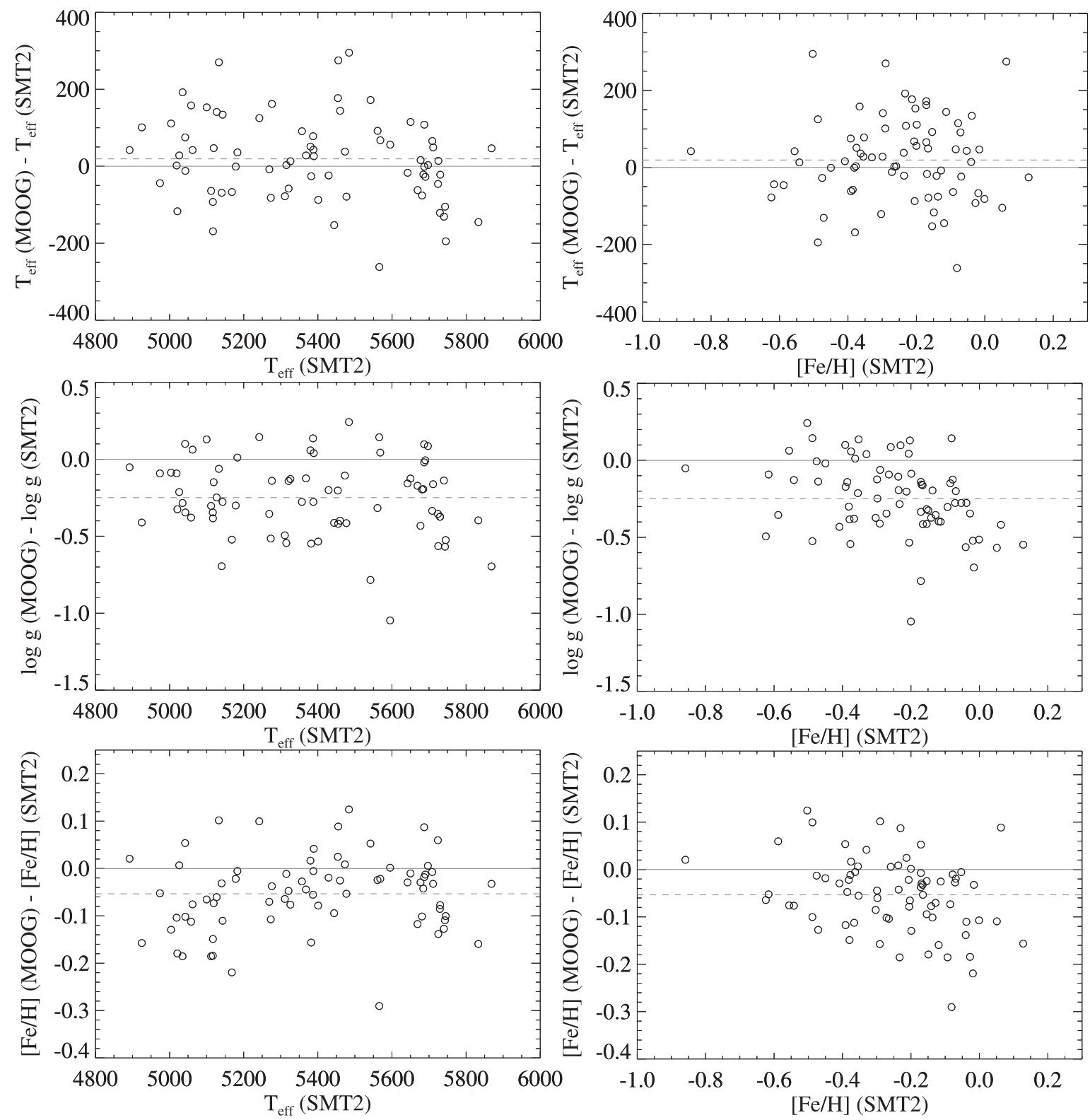

Figure 5. Same as in Figure 4, but comparisons with SMT analysis based on IRFM temperatures (SMT2).

$\overline{\delta M_{V}}$ can essentially be treated as a function of metallicity alone. Theoretical predictions can be used to derive a metallicity term in broadband colors, but they are still uncertain because of the large remaining uncertainties in input physics and stellar model parameters; ultimately they need to be constrained against a well-defined set of observational data (e.g., An et al. 2007a, 2007b, 2015). Comparisons of our sample stars with theoretical models will be discussed in the next paper of this series.

To derive the metallicity correction term in Equation (3) on an empirical basis, we employed the Hipparcos data. Here, our core assumption is that parallaxes for the majority of stars in the Hipparcos catalog are correct, but only a small fraction of these stars, such as those in the Pleiades, have larger parallax errors than those specified in the catalog. For this, we computed a difference between $M_{V}$ from Hipparcos and $M_{V}$ from the
Hyades' MS at a given color of a star:

$$
\begin{gathered}
\delta M_{V, i} \equiv M_{V, i}^{\mathrm{HIP}}-M_{V}^{\text {Hyades }}\left(X_{i}\right) \\
=V_{i}+5 \log \pi_{i}+5-M_{V}^{\text {Hyades }}\left(X_{i}\right),
\end{gathered}
$$

where $V_{i}$ and $\pi_{i}$ are an observed $V$ magnitude and a star's parallax, respectively. The average $\delta M_{V}$ for a sufficiently large number of stars in the narrow bin of metallicity is

$$
\overline{\delta M_{V}}([\mathrm{Fe} / \mathrm{H}])=\left\langle M_{V}^{\mathrm{HIP}}\right\rangle-M_{V}^{\text {Hyades }},
$$

and yields an empirical correction to be added to the $M_{V}^{\text {Hyades }}$ of a star (Equation (3)). The $\overline{\delta M_{V}}$ can be computed for various bins in $[\mathrm{Fe} / \mathrm{H}]$, and these corrections can be expressed as a metallicity sensitivity function in a given color index.

Using Equations (3) and (4), the difference between the MSfitting and Hipparcos-based distance in Equation (1) can be 

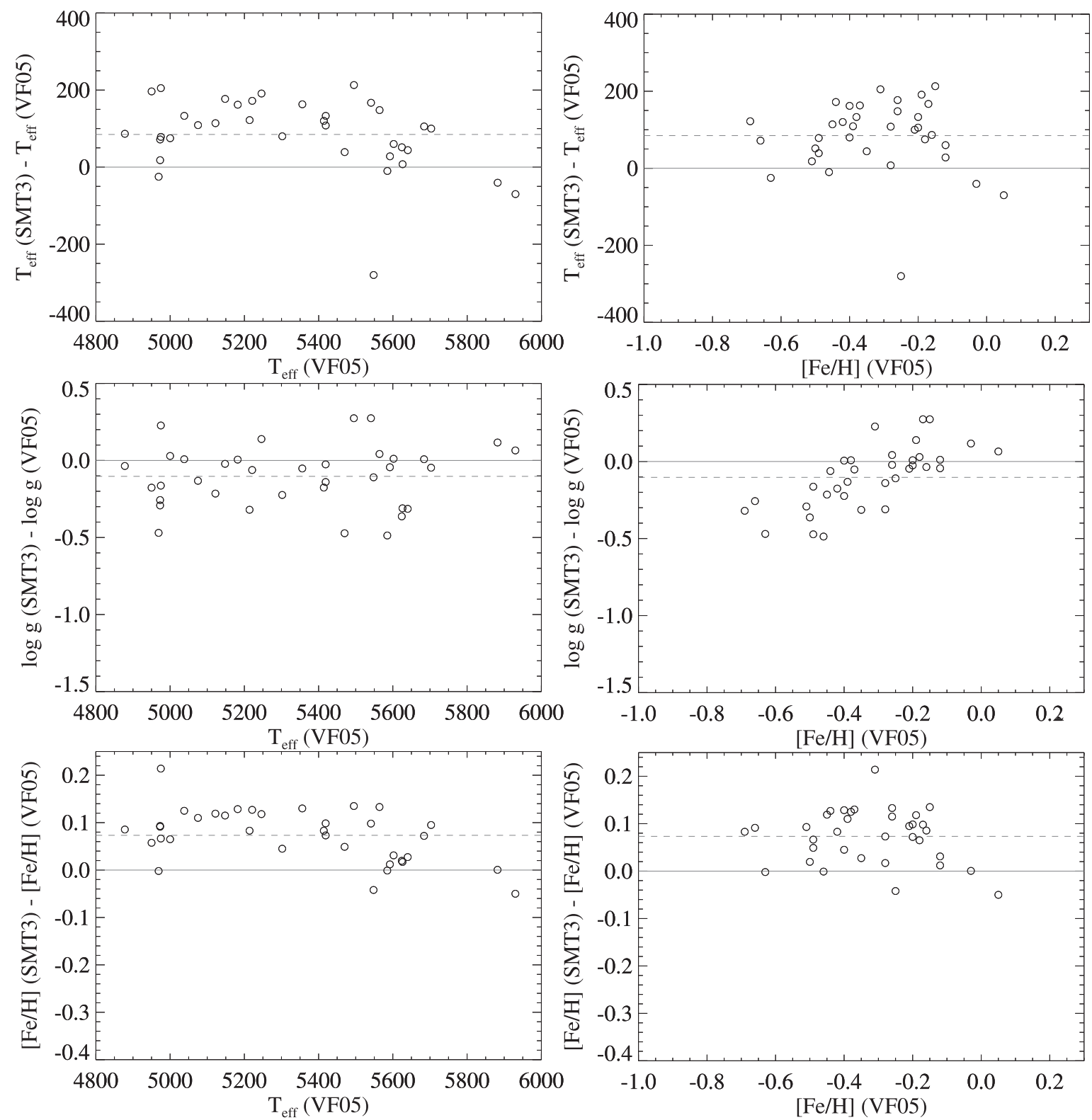

Figure 6. Same as in Figure 4, but showing comparisons between SMT3 and VF05. In the bottom panels, metallicities from SMT3, represent raw estimates before applying a zero-point adjustment (see text).

expressed as

$$
\begin{gathered}
\Delta M_{V, i} \equiv M_{V, i}^{\mathrm{HIP}}-M_{V}^{\mathrm{Hyades}}\left(X_{i}\right)-\overline{\delta M_{V}}\left([\mathrm{Fe} / \mathrm{H}]_{i}\right) \\
=\delta M_{V, i}-\overline{\delta M_{V}}\left([\mathrm{Fe} / \mathrm{H}]_{i}\right) .
\end{gathered}
$$

Because the metallicity correction term $\overline{\delta M_{V}}([\mathrm{Fe} / \mathrm{H}])$ depends only on metallicity, searching for the anomalously subluminous stars with large differences in $M_{V}$ between the MSfitting and the Hipparcos distance is equivalent to finding outliers in $\delta M_{V}$ at a given metallicity.

We utilized stars in VF05 with good parallaxes $\left(\sigma_{\pi} / \pi \leqslant 0.03\right)$ to derive an empirical metallicity sensitivity function, or the amount of offset in $M_{V}$ as a function of metallicity, in broadband colors. We restricted the sample to $0.7 \leqslant B-V \leqslant 1.0$ to make the sensitivity measurement suitable for our KPNO targets. The (conservative) lower limit
$(B-V=0.7)$ was set to eliminate potential contaminations by bright turn-off stars. The top panel in Figure 8 shows $\delta M_{V}$ of these stars computed using Equation (5) in $B-V$ colors. These values decrease toward higher metallicity, because metal-rich MS stars are brighter than metal-poor stars at a given $B-V$ color. The observed trend in this panel reflects the metallicity sensitivity function (Equation (6)).

The observed metallicity sensitivity seen in the top panel of Figure 8 tends to become steeper at higher metallicities. On the other hand, our sample is restricted to those having metallicities below solar, where the observed trend can be approximated by a straight line. Another consideration when deriving a metallicity sensitivity function is that $M_{V}$ estimated using Hyades' MS $\left(M_{V}^{\text {Hyades }}\right)$ must be equal to $M_{V}$ from Hipparcos $\left(M_{V}^{\mathrm{HIP}}\right)$ at the metallicity of the Hyades $([\mathrm{Fe} / \mathrm{H}]=+0.13)$, or $\delta M_{V}=0$, by definition. However, more stars are found 

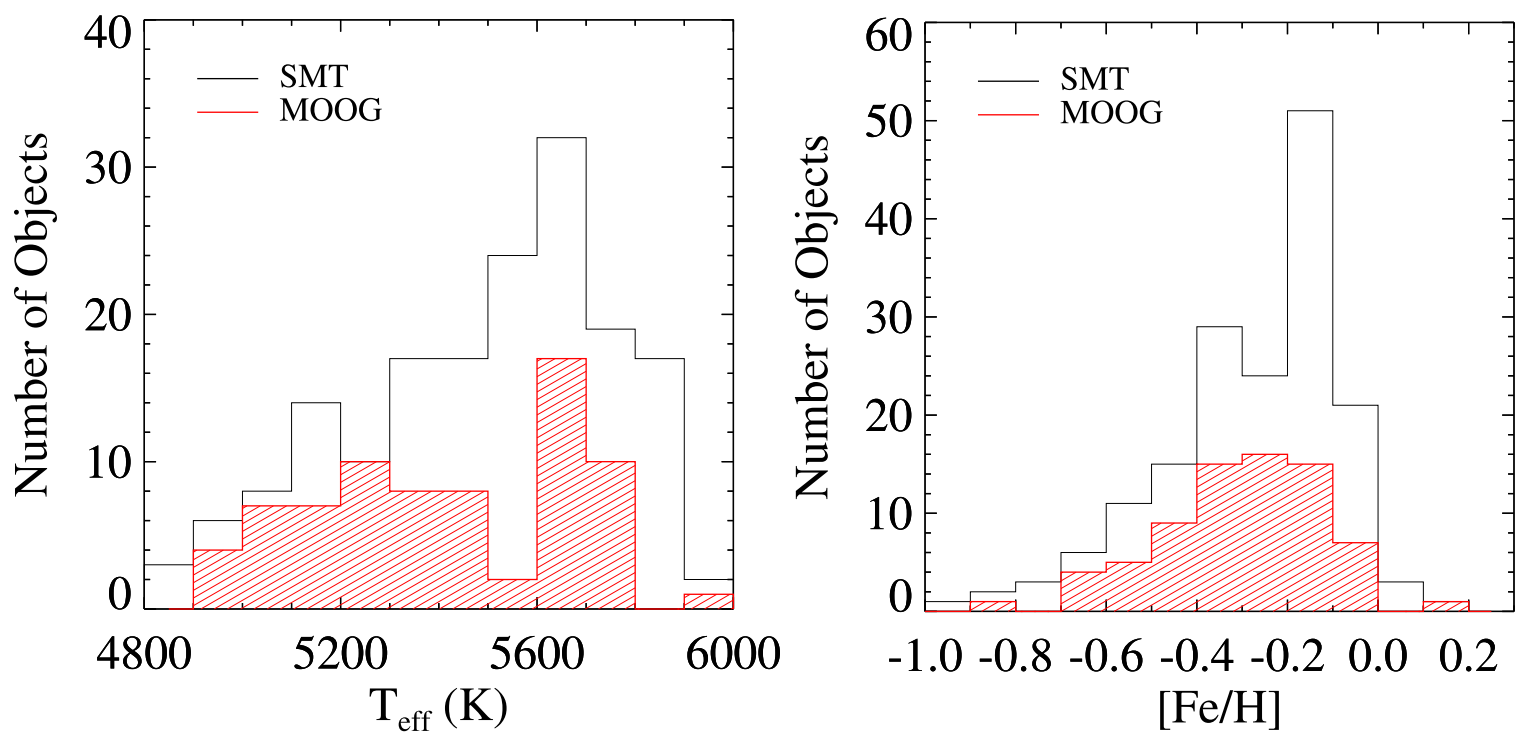

Figure 7. Distributions of effective temperature (left) and metallicity (right) of the KPNO samples as obtained using SMT3 (black histogram) and those from MOOG (red shaded histogram). Metallicities are those after applying a zero-point adjustment.
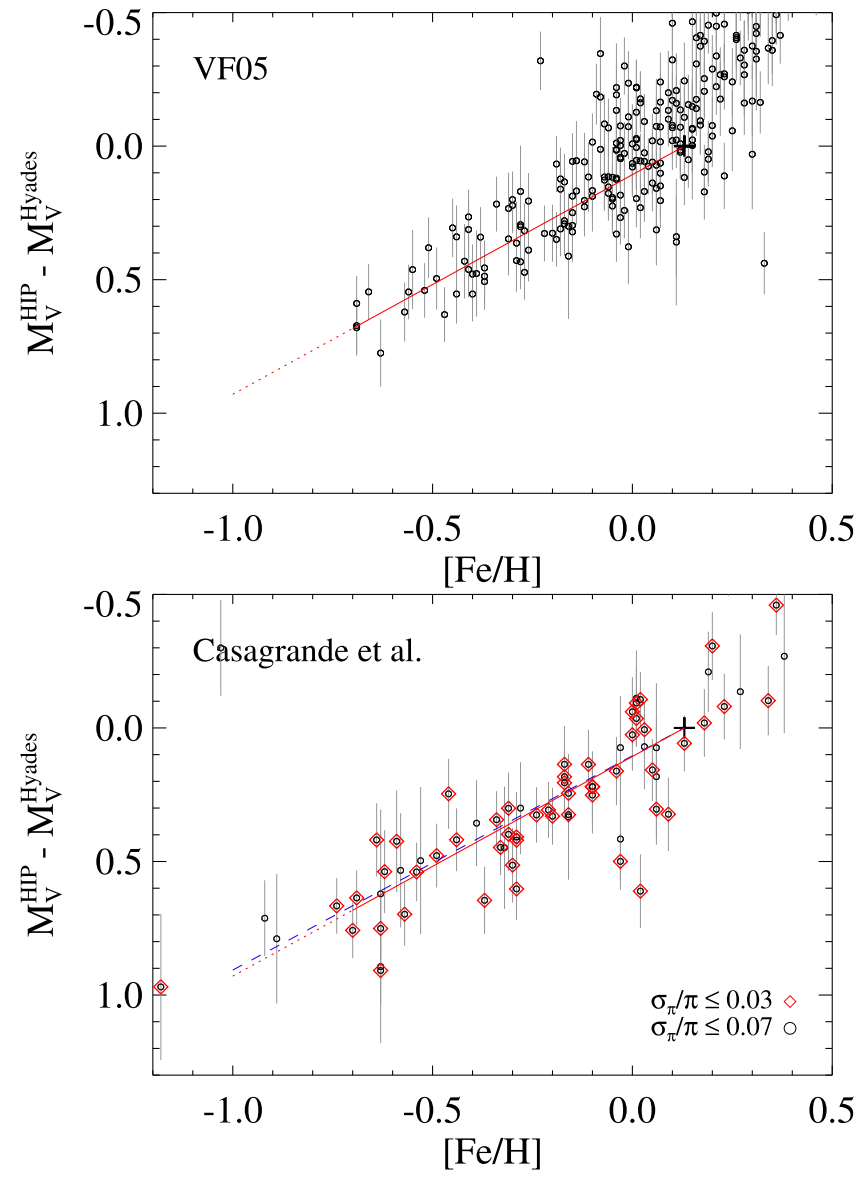

Figure 8. Top: the $\delta M_{V}$ of the Hipparcos field dwarfs with metallicities from VF05. Only those in $0.7 \leqslant B-V \leqslant 1.0$ with good parallaxes $\left(\sigma_{\pi} / \pi \leqslant 0.03\right)$ are shown. The red solid line is a linear fit to stars in $-0.7 \leqslant[\mathrm{Fe} / \mathrm{H}] \leqslant-0.1$, forced to match the position of the Hyades at $[\mathrm{Fe} / \mathrm{H}]=+0.13$ with a zero magnitude difference (black cross), and is shown in both panels. Bottom: same as in the top panel, but showing calibration stars in Casagrande et al. (2010). The blue dashed line is a linear fit to stars with fractional errors in parallax of better than $3 \%$ (red diamond points), which passes through the black cross. with negative $\delta M_{V}$ than those with positive values at $[\mathrm{Fe} / \mathrm{H}] \sim+0.13$. This could be due to a scale error in metallicity or the presence of unresolved binaries in the sample, which are brighter than single MS stars, and therefore have systematically smaller $\delta M_{V}$. Without having a complete census on binarity, we simply proceeded with fitting a straight line to the data for stars with $-1.0 \leqslant[\mathrm{Fe} / \mathrm{H}] \leqslant-0.1$, as shown by the red line, forced to pass through our adopted reference point, the Hyades (cross mark). Our metallicity sensitivity line has a slope of $-0.8 \mathrm{mag} \mathrm{dex}^{-1}$, and is used in the following analysis as a reference value for the mean metallicity sensitivity function in $B-V$.

We additionally used stars in Casagrande et al. (2010) to independently check this metallicity sensitivity function. We cross-identified stars in Casagrande et al. with the Hipparcos catalog using a $10^{\prime \prime}$ search radius along with stellar colors and magnitudes. In the bottom panel of Figure 8, stars with fractional errors in parallax of better than $3 \%$ are shown with a diamond and those with $\leqslant 7 \%$ as circled points, with spectroscopic metallicities as reported in Casagrande et al. (2010). As in the top panel, we selected stars in $0.7 \leqslant B-V \leqslant 1.0$. The blue dashed line is a linear fit to stars in Casagrande et al. with $\leqslant 3 \%$ fractional errors in parallax (red diamond points), and shows nearly the same slope as the one obtained using stars in VF05 (red solid line).

In Figure 8 , the dispersion of the data points around the mean line is $\sigma\left(\delta M_{V}\right)=0.12 \mathrm{mag}$ for VF05 and 0.10 mag for Casagrande et al. (2010), when only stars with good parallaxes $\left(\sigma_{\pi} / \pi \leqslant 0.03\right)$ are used. Photometric errors in $B-V$ of $\sim 0.02 \mathrm{mag}$, which are reasonable to assume, are translated into $0.1 \mathrm{mag}$ error in $M_{V}$, because the slope of MS is about 5 on a $B-V$ CMD. The error in the observed $V$ magnitude directly affects the error in $\delta M_{V}$ (see Equation (5)), but is negligible compared to that from a color error. A parallax error likely produces an error in $\delta M_{V}$ of $\sim 0.05 \mathrm{mag}$, and the error in $[\mathrm{Fe} / \mathrm{H}]$ of $\sim 0.05 \mathrm{dex}$ is translated into $0.04 \mathrm{mag}$ error in $\delta M_{V}$. All together, the error in $\delta M_{V}$ is largely dominated by photometric color errors. The remaining errors could come from unresolved companions of binaries and/or an older age of a star than the 


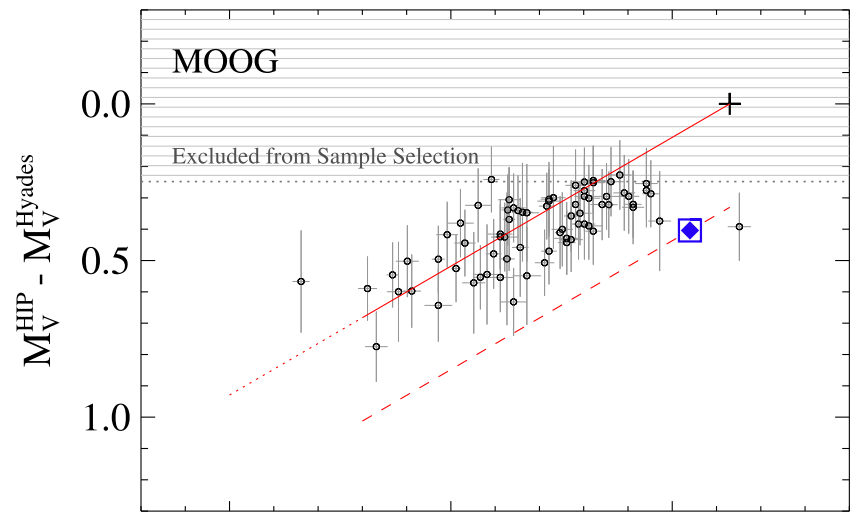

$\begin{array}{lll}-1.0 & -0.5 & 0.0\end{array}$ $[\mathrm{Fe} / \mathrm{H}]$

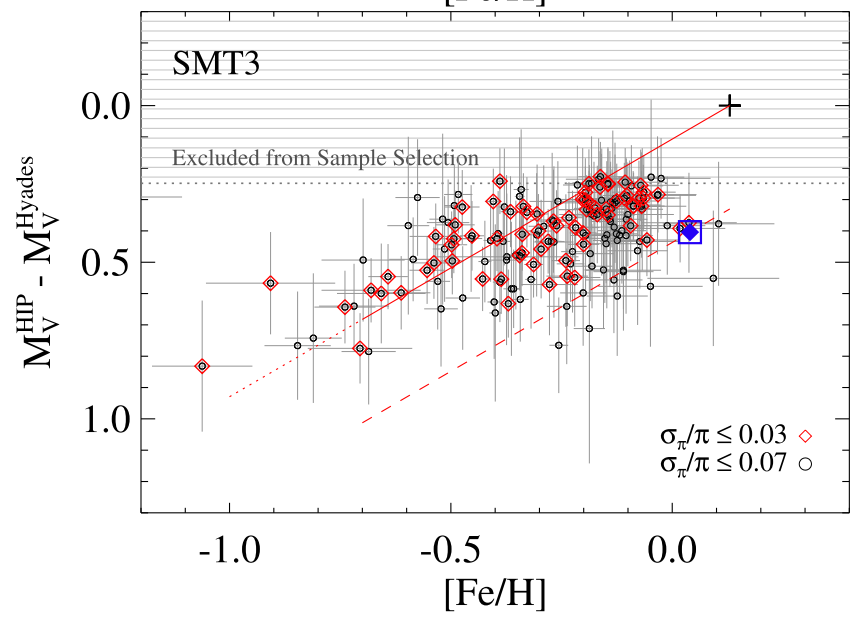

Figure 9. $\delta M_{V}$ of the KPNO sample with MOOG (top) and SMT3 (bottom) metallicity estimates. Stars in the top panel are those having good parallax measurements $\left(\sigma_{\pi} / \pi \leqslant 0.03\right)$. The same set of stars is also shown as red diamond points in the bottom panel. The red solid line represents a mean metallicity sensitivity function as derived from the VF05 stars in the top panel of Figure 8. The blue diamond point with a box indicates Pleiades, and its magnitude excess $\left(\Delta M_{V}\right)$ is shown by a red dashed line. The gray shaded area represents the $\delta M_{V}$ limit set by our sample selection.

Hyades (550 Myr), which would make stars look brighter than a single-star zero-age MS.

While the observed dispersion is close to our expectation, there are a few outliers in Figure 8 that are far below the metallicity sensitivity line, with the largest magnitude offset $\Delta M_{V}$ of about $0.5 \mathrm{mag}$. According to Equation (8), this offset corresponds to a larger Hipparcos-based absolute magnitude than $M_{V}$ from MS fitting. One possibility is that the $B-V$ color of a star is too blue by $0.1 \mathrm{mag}$, or that $V$ mag is too large by $0.5 \mathrm{mag}$, but the sizes of photometric errors required are uncomfortably large. The other possibility is that a star's spectroscopic metallicity was overestimated by $\Delta[\mathrm{Fe} / \mathrm{H}]=0.5$, but again the expected error seems too large for a high-resolution spectroscopic analysis. In addition, this can be due to an overestimated parallax measurement in the Hipparcos catalog, which may pose a similar problem with the Pleiades stars. However, a parallax error cannot be condemned for a large $\Delta M_{V}$ unless other sources of errors are well understood. In the following analysis, we used our homogeneous KPNO data set with well-determined spectroscopic metallicities to better identify and characterize the properties of outliers in the $\delta M_{V}$ versus $[\mathrm{Fe} / \mathrm{H}]$ diagram.

\subsection{Magnitude Excess of KPNO Sample}

Figure 9 shows the same $\delta M_{V}$ versus $[\mathrm{Fe} / \mathrm{H}]$ plot as in Figure 8 , but for our KPNO sample, where $\delta M_{V}$ of stars and their errors are those listed in the second and third columns in Table 7. Stars with MOOG and SMT3 metallicities (both scaled to match the VF05 metallicities) are used in the top and the bottom panel, respectively. In each panel, the cross mark indicates the Hyades at the cluster's distance from Hipparcos $\left(\Delta M_{V}=\delta M_{V}=0\right)$, and the same red line as in the top panel of Figure 8 is shown for the empirical metallicity sensitivity function in $B-V$ CMDs. A magnitude excess in this study is defined as having a shorter Hipparcos distance than that from MS fitting, or a positive offset of a star from the mean metallicity function $\left(\Delta M_{V}>0\right)$. While our sample does not include stars in the Pleiades, we mark the position of the Pleiades as a blue diamond point at its well-known metallicity $[\mathrm{Fe} / \mathrm{H}]=+0.04$ (see references in An et al. 2007b). We found $\delta M_{V}=0.40$ from 33 single MS stars in the Pleiades using the Hyades' MS. The Hipparcos distance of the Pleiades is shorter than the MSfitting distance by $\Delta(m-M)_{0}=\Delta M_{V}=0.33$ (Pinsonneault et al. 1998; An et al. 2007b). Below we use $\Delta M_{V}=0.33$ as a reference to judge whether stars in our KPNO sample are anomalously faint, or have a large magnitude excess, like those in the Pleiades at its Hipparcos distance.

In the top panel of Figure 9, a total of 74 field stars with MOOG metallicities are shown, representing the entire KPNO sample with parallax errors of better than $3 \%$. In the bottom panel, 167 sample stars with SMT3 metallicities are shown with parallax errors of better than $7 \%$. The red diamond points are a subset of these stars, having more accurate parallaxes $\left(\sigma_{\pi} / \pi \leqslant 0.03\right)$. The vertical error bars are a quadrature sum of errors from photometry and parallax (see Equation (5)). As in Figure 8, we included an error in $\delta M_{V}$ from a photometric color by multiplying a $B-V$ error by a slope of the Hyades' MS. In the bottom panel, horizontal error bars are metallicity errors reported by SMT; HIP 95727 is not displayed because of its large metallicity error (0.51 dex).

In Figure 9 the gray shaded region represents a forbidden area that was excluded from our color-magnitude selection of the KPNO sample. As shown in Figure 1, the upper limit in stellar brightness (or a lower limit in $M_{V}$ ) in our sample selection was set almost parallel to the MS of the Hyades, and any stars having $\delta M_{V} \lesssim 0.25 \mathrm{mag}$ were not included in our observing runs. The boundary of the shaded region is not a clearcut division, because the slope of the Hyades' MS is not exactly parallel to our color-magnitude selection. Nevertheless, our search for sub-luminous MS stars is almost unaffected by this sample bias because such stars would reveal themselves as having significantly faint $M_{V}$ with the Hipparcos parallax, or a large $\Delta M_{V}$ at a given metallicity. On the other hand, a metallicity sensitivity function cannot be constructed using our field star sample alone, because of a sample bias against intrinsically bright, metal-rich stars.

Considering this sample bias, most of our KPNO stars in Figure 9 are distributed in the same manner as in Figure 8 and follow the empirical metallicity sensitivity function (red solid line). In the bottom panel, a distribution of the sample with $\leqslant 3 \%$ parallax errors is tighter than that from the entire KPNO sample. The true dispersion of a magnitude excess from the mean sensitivity line $\left[\sigma\left(\Delta M_{V}\right)\right]$ is difficult to measure for our sample due to the limit set by our sample selection (gray region). Nevertheless, metal-poor stars 
Table 7

$\delta M_{V}, \Delta M_{V}$, and Lithium EWs of the KPNO Sample

\begin{tabular}{|c|c|c|c|c|c|c|c|c|c|}
\hline \multirow[b]{3}{*}{ HIP } & \multicolumn{6}{|c|}{$B-V$} & \multicolumn{2}{|c|}{$V-K_{s}$} & \multirow[b]{3}{*}{$\begin{array}{l}\mathrm{W}(\mathrm{Li}) \\
(\mathrm{m} \AA)\end{array}$} \\
\hline & \multirow[b]{2}{*}{$\begin{array}{c}\delta M_{V} \\
(\mathrm{mag})\end{array}$} & \multirow[b]{2}{*}{$\begin{array}{c}\sigma\left(\delta M_{V}\right) \\
(\mathrm{mag})\end{array}$} & \multicolumn{2}{|c|}{ SMT3 } & \multicolumn{2}{|c|}{ MOOG } & \multirow[b]{2}{*}{$\begin{array}{c}\delta M_{V} \\
(\mathrm{mag})\end{array}$} & \multirow[b]{2}{*}{$\begin{array}{c}\sigma\left(\delta M_{V}\right) \\
(\mathrm{mag})\end{array}$} & \\
\hline & & & $\begin{array}{l}\Delta M_{V} \\
(\mathrm{mag})\end{array}$ & $\begin{array}{c}\sigma\left(\Delta M_{V}\right) \\
(\mathrm{mag})\end{array}$ & $\begin{array}{l}\Delta M_{V} \\
(\mathrm{mag})\end{array}$ & $\begin{array}{c}\sigma\left(\Delta M_{V}\right) \\
(\mathrm{mag})\end{array}$ & & & \\
\hline 1674 & 0.43 & 0.12 & 0.01 & 0.12 & $\ldots$ & & -0.93 & 0.09 & $\ldots$ \\
\hline 4907 & 0.32 & 0.11 & 0.14 & 0.13 & 0.14 & 0.11 & 0.26 & 0.07 & $\ldots$ \\
\hline 5313 & 0.77 & 0.15 & 0.45 & 0.15 & 0.48 & 0.15 & 0.51 & 0.13 & 80.20 \\
\hline 5521 & 0.32 & 0.11 & 0.14 & 0.13 & 0.03 & 0.11 & 0.21 & 0.08 & 12.17 \\
\hline 9172 & 0.28 & 0.12 & 0.12 & 0.14 & 0.12 & 0.12 & $\ldots$ & $\ldots$ & 69.40 \\
\hline 9829 & 0.32 & 0.11 & -0.06 & 0.11 & 0.10 & 0.11 & 0.14 & 0.06 & $\ldots$ \\
\hline 10276 & 0.56 & 0.17 & 0.34 & 0.18 & 0.30 & 0.17 & 0.37 & 0.10 & $\ldots$ \\
\hline 10629 & 0.32 & 0.13 & -0.19 & 0.13 & $\ldots$ & $\ldots$ & 0.06 & 0.10 & $\ldots$ \\
\hline 11507 & 0.43 & 0.18 & 0.22 & 0.18 & $\ldots$ & $\ldots$ & 0.22 & 0.11 & $\ldots$ \\
\hline 12685 & 0.33 & 0.14 & 0.07 & 0.16 & $\ldots$ & $\ldots$ & -0.07 & 0.12 & 75.40 \\
\hline 13771 & 0.41 & 0.20 & 0.05 & 0.20 & $\ldots$ & $\ldots$ & 0.04 & 0.14 & $\ldots$ \\
\hline 14241 & 0.46 & 0.17 & -0.07 & 0.17 & $\ldots$ & $\ldots$ & 0.11 & 0.10 & $\ldots$ \\
\hline 14300 & 0.25 & 0.13 & -0.03 & 0.13 & $\ldots$ & $\ldots$ & 0.27 & 0.09 & $\ldots$ \\
\hline 15062 & 0.56 & 0.13 & 0.14 & 0.13 & $\ldots$ & $\ldots$ & 0.24 & 0.10 & 32.50 \\
\hline 15442 & 0.25 & 0.11 & -0.01 & 0.12 & -0.02 & 0.11 & 0.15 & 0.07 & 13.83 \\
\hline 17265 & 0.60 & 0.16 & -0.05 & 0.19 & -0.02 & 0.16 & 0.23 & 0.09 & $\ldots$ \\
\hline 18324 & 0.38 & 0.11 & -0.13 & 0.12 & -0.12 & 0.11 & 0.11 & 0.07 & $\ldots$ \\
\hline 20722 & 0.23 & 0.13 & 0.10 & 0.17 & $\ldots$ & $\ldots$ & 0.13 & 0.10 & 24.90 \\
\hline 21276 & 0.30 & 0.12 & 0.13 & 0.15 & 0.11 & 0.12 & -0.10 & 0.08 & 74.30 \\
\hline 21571 & 0.36 & 0.11 & 0.06 & 0.12 & 0.06 & 0.12 & 0.17 & 0.08 & $\ldots$ \\
\hline 21832 & 0.50 & 0.11 & -0.05 & 0.13 & -0.10 & 0.12 & 0.01 & 0.08 & $\ldots$ \\
\hline 22175 & 0.33 & 0.12 & 0.10 & 0.14 & 0.15 & 0.12 & 0.23 & 0.08 & $\ldots$ \\
\hline 23431 & 0.37 & 0.14 & -0.15 & 0.15 & $\ldots$ & $\ldots$ & 0.23 & 0.11 & $\ldots$ \\
\hline 23786 & 0.30 & 0.11 & 0.10 & 0.12 & 0.03 & 0.11 & 0.05 & 0.08 & $\ldots$ \\
\hline 26505 & 0.50 & 0.11 & -0.02 & 0.11 & -0.05 & 0.11 & 0.16 & 0.08 & $\ldots$ \\
\hline 30862 & 0.53 & 0.11 & -0.04 & 0.11 & 0.02 & 0.11 & 0.29 & 0.07 & 15.31 \\
\hline 44259 & 0.58 & 0.21 & 0.18 & 0.21 & $\ldots$ & $\ldots$ & 0.15 & 0.09 & $\ldots$ \\
\hline 44262 & 0.23 & 0.25 & 0.08 & 0.26 & $\ldots$ & $\ldots$ & 0.14 & 0.16 & $\ldots$ \\
\hline 52278 & 0.28 & 0.12 & -0.22 & 0.13 & $\ldots$ & $\ldots$ & 0.36 & 0.09 & $\ldots$ \\
\hline 52470 & 0.42 & 0.11 & -0.06 & 0.11 & -0.01 & 0.11 & 0.31 & 0.08 & $\ldots$ \\
\hline 54906 & 0.39 & 0.11 & 0.10 & 0.12 & 0.13 & 0.11 & 0.26 & 0.07 & $\ldots$ \\
\hline 55210 & 0.33 & 0.11 & 0.08 & 0.11 & -0.01 & 0.11 & 0.11 & 0.08 & $\ldots$ \\
\hline 56092 & 0.44 & 0.24 & 0.11 & 0.25 & $\ldots$ & $\ldots$ & 0.47 & 0.15 & $\ldots$ \\
\hline 56337 & 0.41 & 0.19 & 0.21 & 0.19 & $\ldots$ & $\ldots$ & 0.31 & 0.12 & $\ldots$ \\
\hline 56837 & 0.58 & 0.13 & 0.18 & 0.13 & $\ldots$ & $\ldots$ & $\ldots$ & $\ldots$ & $\ldots$ \\
\hline 57939 & 1.37 & 0.10 & 0.12 & 0.22 & 0.15 & 0.10 & 0.67 & 0.07 & $\ldots$ \\
\hline 57992 & 0.63 & 0.11 & 0.22 & 0.11 & 0.23 & 0.11 & 0.34 & 0.07 & $\ldots$ \\
\hline 58949 & 0.40 & 0.12 & 0.04 & 0.12 & 0.09 & 0.12 & 0.08 & 0.08 & $\ldots$ \\
\hline 60074 & 0.29 & 0.11 & 0.10 & 0.12 & 0.14 & 0.11 & 0.17 & 0.06 & 125.50 \\
\hline 60268 & 0.64 & 0.12 & -0.07 & 0.13 & 0.10 & 0.12 & 0.08 & 0.08 & $\ldots$ \\
\hline 63322 & 0.42 & 0.25 & 0.22 & 0.26 & $\ldots$ & $\ldots$ & -0.34 & 0.16 & 121.40 \\
\hline 63346 & 0.43 & 0.11 & -0.09 & 0.12 & 0.01 & 0.11 & 0.12 & 0.07 & $\ldots$ \\
\hline 63636 & 0.24 & 0.11 & 0.05 & 0.14 & -0.01 & 0.11 & 0.24 & 0.07 & 25.55 \\
\hline 64076 & 0.51 & 0.12 & 0.21 & 0.13 & $\ldots$ & $\ldots$ & 0.21 & 0.09 & 13.04 \\
\hline 64131 & 0.64 & 0.14 & -0.06 & 0.16 & $\ldots$ & $\ldots$ & 0.36 & 0.11 & $\ldots$ \\
\hline 65165 & 0.25 & 0.11 & 0.09 & 0.14 & 0.10 & 0.12 & 0.22 & 0.08 & $\ldots$ \\
\hline 65352 & 0.48 & 0.11 & 0.09 & 0.11 & 0.04 & 0.11 & 0.38 & 0.07 & $\ldots$ \\
\hline 65355 & 0.34 & 0.11 & -0.04 & 0.12 & -0.05 & 0.12 & 0.30 & 0.08 & $\ldots$ \\
\hline 65935 & 0.46 & 0.24 & 0.29 & 0.25 & $\ldots$ & $\ldots$ & 0.27 & 0.14 & $\ldots$ \\
\hline 66509 & 0.49 & 0.20 & -0.19 & 0.22 & $\ldots$ & $\ldots$ & 0.07 & 0.14 & $\ldots$ \\
\hline 67211 & 0.49 & 0.27 & 0.08 & 0.28 & $\ldots$ & $\ldots$ & 0.18 & 0.13 & $\ldots$ \\
\hline 67282 & 0.32 & 0.17 & 0.15 & 0.17 & $\ldots$ & $\ldots$ & 0.26 & 0.09 & $\ldots$ \\
\hline 67773 & 0.43 & 0.13 & 0.10 & 0.13 & $\ldots$ & $\ldots$ & 0.19 & 0.09 & $\ldots$ \\
\hline 67904 & 0.34 & 0.11 & -0.07 & 0.12 & -0.07 & 0.11 & 0.33 & 0.08 & $\ldots$ \\
\hline 70253 & 0.60 & 0.12 & -0.01 & 0.13 & 0.01 & 0.12 & 0.21 & 0.08 & $\ldots$ \\
\hline 70319 & 0.42 & 0.10 & -0.01 & 0.10 & -0.00 & 0.10 & 0.04 & 0.06 & $\ldots$ \\
\hline 71720 & 0.31 & 0.23 & 0.10 & 0.24 & $\ldots$ & $\ldots$ & 0.41 & 0.13 & $\ldots$ \\
\hline 72577 & 0.41 & 0.23 & 0.18 & 0.24 & $\ldots$ & $\ldots$ & 0.20 & 0.14 & $\ldots$ \\
\hline 72663 & 0.39 & 0.19 & 0.04 & 0.20 & $\ldots$ & $\ldots$ & 0.23 & 0.14 & $\ldots$ \\
\hline 72703 & 0.39 & 0.17 & 0.17 & 0.18 & $\ldots$ & $\ldots$ & 0.30 & 0.09 & 87.00 \\
\hline 73005 & 0.31 & 0.10 & -0.13 & 0.11 & -0.10 & 0.11 & 0.17 & 0.06 & $\ldots$ \\
\hline
\end{tabular}


Table 7

(Continued)

\begin{tabular}{|c|c|c|c|c|c|c|c|c|c|}
\hline \multirow[b]{3}{*}{ HIP } & \multicolumn{6}{|c|}{$B-V$} & \multicolumn{2}{|c|}{$V-K_{s}$} & \multirow[b]{3}{*}{$\begin{array}{l}\mathrm{W}(\mathrm{Li}) \\
(\mathrm{m} \AA)\end{array}$} \\
\hline & \multirow[b]{2}{*}{$\begin{array}{c}\delta M_{V} \\
(\mathrm{mag})\end{array}$} & \multirow[b]{2}{*}{$\begin{array}{c}\sigma\left(\delta M_{V}\right) \\
(\mathrm{mag})\end{array}$} & \multicolumn{2}{|c|}{ SMT3 } & \multicolumn{2}{|c|}{ MOOG } & \multirow[b]{2}{*}{$\begin{array}{c}\delta M_{V} \\
(\mathrm{mag})\end{array}$} & \multirow[b]{2}{*}{$\begin{array}{c}\sigma\left(\delta M_{V}\right) \\
(\mathrm{mag})\end{array}$} & \\
\hline & & & $\begin{array}{l}\Delta M_{V} \\
(\mathrm{mag})\end{array}$ & $\begin{array}{c}\sigma\left(\Delta M_{V}\right) \\
(\mathrm{mag})\end{array}$ & $\begin{array}{c}\Delta M_{V} \\
(\mathrm{mag})\end{array}$ & $\begin{array}{c}\sigma\left(\Delta M_{V}\right) \\
(\mathrm{mag})\end{array}$ & & & \\
\hline 73138 & 0.58 & 0.19 & 0.43 & 0.23 & 0.47 & 0.19 & 0.28 & 0.14 & $\ldots$ \\
\hline 73677 & 0.49 & 0.13 & -0.10 & 0.14 & $\ldots$ & $\ldots$ & 0.34 & 0.11 & $\ldots$ \\
\hline 73845 & 0.38 & 0.20 & 0.36 & 0.23 & -0.16 & 0.20 & 0.16 & 0.14 & $\ldots$ \\
\hline 74126 & 0.61 & 0.19 & 0.40 & 0.20 & 0.54 & 0.19 & 0.31 & 0.14 & 29.80 \\
\hline 74396 & 0.32 & 0.11 & 0.16 & 0.15 & 0.08 & 0.12 & 0.12 & 0.08 & $\ldots$ \\
\hline 75059 & 0.25 & 0.15 & -0.01 & 0.16 & $\ldots$ & $\ldots$ & 0.35 & 0.13 & $\ldots$ \\
\hline 75370 & 0.42 & 0.17 & -0.06 & 0.17 & $\ldots$ & $\ldots$ & -0.07 & 0.10 & $\ldots$ \\
\hline 75446 & 0.53 & 0.19 & 0.33 & 0.20 & 0.36 & 0.19 & 0.41 & 0.13 & $\ldots$ \\
\hline 75703 & 0.60 & 0.17 & 0.33 & 0.18 & $\ldots$ & $\ldots$ & 0.36 & 0.10 & 14.20 \\
\hline 76058 & 0.47 & 0.19 & 0.17 & 0.20 & $\ldots$ & $\ldots$ & 0.24 & 0.13 & 18.60 \\
\hline 76130 & 0.34 & 0.20 & 0.10 & 0.21 & $\ldots$ & $\ldots$ & 0.30 & 0.15 & 27.10 \\
\hline 76330 & 0.43 & 0.12 & 0.28 & 0.14 & 0.13 & 0.12 & 0.16 & 0.09 & $\ldots$ \\
\hline 76674 & 0.29 & 0.19 & 0.15 & 0.21 & $\ldots$ & $\ldots$ & 0.05 & 0.13 & 71.60 \\
\hline 77810 & 0.43 & 0.15 & 0.27 & 0.18 & $\ldots$ & $\ldots$ & 0.43 & 0.13 & 120.60 \\
\hline 78028 & 0.30 & 0.14 & 0.08 & 0.16 & $\ldots$ & $\ldots$ & 0.14 & 0.12 & $\ldots$ \\
\hline 78241 & 0.55 & 0.11 & 0.13 & 0.11 & 0.13 & 0.11 & 0.26 & 0.07 & $\ldots$ \\
\hline 78336 & 0.55 & 0.22 & 0.52 & 0.26 & 0.46 & 0.22 & 0.17 & 0.10 & 28.10 \\
\hline 78550 & 0.62 & 0.14 & 0.23 & 0.14 & $\ldots$ & $\ldots$ & 0.28 & 0.11 & $\ldots$ \\
\hline 78775 & 0.59 & 0.10 & -0.08 & 0.15 & -0.08 & 0.10 & 0.21 & 0.06 & $\ldots$ \\
\hline 78923 & 0.23 & 0.13 & -0.01 & 0.15 & $\ldots$ & $\ldots$ & 0.17 & 0.10 & $\ldots$ \\
\hline 79629 & 0.47 & 0.11 & 0.08 & 0.11 & 0.13 & 0.11 & 0.05 & 0.07 & $\ldots$ \\
\hline 80243 & 0.37 & 0.16 & 0.30 & 0.19 & 0.24 & 0.16 & 0.11 & 0.07 & $\ldots$ \\
\hline 80262 & 0.30 & 0.13 & 0.12 & 0.14 & $\ldots$ & $\ldots$ & 0.11 & 0.10 & $\ldots$ \\
\hline 81186 & 0.49 & 0.21 & 0.19 & 0.21 & 0.08 & 0.21 & 0.30 & 0.09 & $\ldots$ \\
\hline 81288 & 0.57 & 0.16 & -0.29 & 0.22 & -0.23 & 0.16 & 0.11 & 0.09 & $\ldots$ \\
\hline 81605 & 0.26 & 0.13 & 0.07 & 0.15 & $\ldots$ & $\ldots$ & 0.07 & 0.10 & $\ldots$ \\
\hline 81831 & 0.64 & 0.18 & 0.34 & 0.19 & 0.29 & 0.19 & 0.64 & 0.12 & $\ldots$ \\
\hline 82120 & 0.50 & 0.13 & 0.04 & 0.13 & $\ldots$ & $\ldots$ & 0.17 & 0.10 & $\ldots$ \\
\hline 82210 & 0.33 & 0.12 & 0.11 & 0.15 & $\ldots$ & $\ldots$ & 0.35 & 0.09 & 14.20 \\
\hline 82388 & 0.31 & 0.13 & -0.01 & 0.14 & $\ldots$ & $\ldots$ & 0.25 & 0.10 & 128.70 \\
\hline 82644 & 0.44 & 0.13 & 0.21 & 0.15 & $\ldots$ & $\ldots$ & 0.28 & 0.09 & 54.90 \\
\hline 82712 & 0.48 & 0.13 & 0.10 & 0.13 & $\ldots$ & $\ldots$ & 0.13 & 0.10 & $\ldots$ \\
\hline 83141 & 0.37 & 0.21 & 0.04 & 0.22 & $\ldots$ & $\ldots$ & 0.15 & 0.10 & $\ldots$ \\
\hline 83276 & 0.35 & 0.13 & 0.09 & 0.13 & $\ldots$ & $\ldots$ & 0.14 & 0.10 & 15.54 \\
\hline 83500 & 0.29 & 0.19 & -0.29 & 0.19 & $\ldots$ & $\ldots$ & 0.32 & 0.12 & 32.30 \\
\hline 84520 & 0.26 & 0.11 & 0.02 & 0.13 & -0.03 & 0.12 & 0.25 & 0.08 & 14.20 \\
\hline 85235 & 0.55 & 0.10 & 0.09 & 0.10 & 0.09 & 0.10 & 0.23 & 0.06 & $\ldots$ \\
\hline 85653 & 0.24 & 0.10 & -0.19 & 0.11 & -0.20 & 0.11 & -0.03 & 0.07 & $\ldots$ \\
\hline 86568 & 0.66 & 0.28 & 0.23 & 0.28 & $\ldots$ & $\ldots$ & 0.27 & 0.15 & $\ldots$ \\
\hline 86765 & 0.38 & 0.11 & 0.06 & 0.12 & 0.11 & 0.12 & 0.14 & 0.09 & $\ldots$ \\
\hline 87089 & 0.48 & 0.17 & 0.08 & 0.18 & $\ldots$ & $\ldots$ & +0.00 & 0.10 & $\ldots$ \\
\hline 88166 & 0.74 & 0.21 & -0.03 & 0.22 & $\ldots$ & $\ldots$ & 0.35 & 0.15 & $\ldots$ \\
\hline 88972 & 0.31 & 0.10 & 0.05 & 0.12 & -0.03 & 0.11 & 0.01 & 0.06 & $\ldots$ \\
\hline 90355 & 0.51 & 0.13 & 0.26 & 0.14 & $\ldots$ & $\ldots$ & 0.17 & 0.11 & $\ldots$ \\
\hline 90415 & 0.36 & 0.27 & -0.17 & 0.27 & $\ldots$ & $\ldots$ & 0.29 & 0.13 & $\ldots$ \\
\hline 90708 & 0.31 & 0.19 & 0.10 & 0.21 & $\ldots$ & $\ldots$ & 0.25 & 0.13 & $\ldots$ \\
\hline 91364 & 0.33 & 0.11 & 0.07 & 0.13 & -0.07 & 0.11 & 0.15 & 0.07 & $\ldots$ \\
\hline 91381 & 0.43 & 0.13 & -0.01 & 0.13 & $\ldots$ & $\ldots$ & 0.17 & 0.10 & $\ldots$ \\
\hline 91438 & 0.44 & 0.10 & 0.17 & 0.11 & 0.14 & 0.10 & $\ldots$ & $\ldots$ & $\ldots$ \\
\hline 91605 & 0.57 & 0.16 & 0.24 & 0.17 & 0.10 & 0.16 & 0.23 & 0.08 & $\ldots$ \\
\hline 91614 & 0.27 & 0.19 & -0.12 & 0.19 & $\ldots$ & $\ldots$ & 0.24 & 0.14 & $\ldots$ \\
\hline 91905 & 0.46 & 0.16 & 0.11 & 0.16 & 0.07 & 0.16 & 0.28 & 0.07 & $\ldots$ \\
\hline 92388 & 0.61 & 0.17 & 0.12 & 0.17 & $\ldots$ & $\ldots$ & 0.20 & 0.09 & $\ldots$ \\
\hline 92569 & 0.28 & 0.11 & 0.01 & 0.12 & 0.01 & 0.11 & 0.11 & 0.07 & $\ldots$ \\
\hline 93195 & 0.28 & 0.12 & 0.15 & 0.15 & 0.09 & 0.12 & 0.13 & 0.09 & $\ldots$ \\
\hline 93731 & 0.53 & 0.12 & 0.33 & 0.15 & $\ldots$ & $\ldots$ & 0.35 & 0.09 & $\ldots$ \\
\hline 94582 & 0.36 & 0.22 & 0.14 & 0.22 & $\ldots$ & $\ldots$ & 0.31 & 0.10 & $\ldots$ \\
\hline 95727 & 0.48 & 0.20 & 0.07 & 0.26 & $\ldots$ & $\ldots$ & 0.27 & 0.15 & $\ldots$ \\
\hline 96100 & 0.35 & 0.10 & 0.12 & 0.12 & 0.07 & 0.11 & $\ldots$ & $\ldots$ & $\ldots$ \\
\hline 96344 & 0.38 & 0.28 & -0.21 & 0.29 & $\ldots$ & $\ldots$ & 0.51 & 0.15 & $\ldots$ \\
\hline
\end{tabular}


Table 7

(Continued)

\begin{tabular}{|c|c|c|c|c|c|c|c|c|c|}
\hline \multirow[b]{3}{*}{ HIP } & \multicolumn{6}{|c|}{$B-V$} & \multicolumn{2}{|c|}{$V-K_{s}$} & \multirow[b]{3}{*}{$\begin{array}{l}\mathrm{W}(\mathrm{Li}) \\
(\mathrm{m} \AA)\end{array}$} \\
\hline & \multirow[b]{2}{*}{$\begin{array}{c}\delta M_{V} \\
(\mathrm{mag})\end{array}$} & \multirow[b]{2}{*}{$\begin{array}{c}\sigma\left(\delta M_{V}\right) \\
(\mathrm{mag})\end{array}$} & \multicolumn{2}{|c|}{ SMT3 } & \multicolumn{2}{|c|}{ MOOG } & \multirow[b]{2}{*}{$\begin{array}{c}\delta M_{V} \\
(\mathrm{mag})\end{array}$} & \multirow[b]{2}{*}{$\begin{array}{c}\sigma\left(\delta M_{V}\right) \\
(\mathrm{mag})\end{array}$} & \\
\hline & & & $\begin{array}{c}\Delta M_{V} \\
\text { (mag) }\end{array}$ & $\begin{array}{c}\sigma\left(\Delta M_{V}\right) \\
(\mathrm{mag})\end{array}$ & $\begin{array}{l}\Delta M_{V} \\
(\mathrm{mag})\end{array}$ & $\begin{array}{c}\sigma\left(\Delta M_{V}\right) \\
(\mathrm{mag})\end{array}$ & & & \\
\hline 96634 & 0.35 & 0.17 & 0.10 & 0.18 & $\ldots$ & $\ldots$ & -0.01 & 0.10 & $\ldots$ \\
\hline 96735 & 0.41 & 0.17 & 0.20 & 0.18 & $\ldots$ & $\ldots$ & 0.04 & 0.09 & $\ldots$ \\
\hline 97640 & 0.29 & 0.11 & -0.91 & 0.25 & $\ldots$ & $\ldots$ & -0.37 & 0.07 & $\ldots$ \\
\hline 97668 & 0.71 & 0.43 & 0.45 & 0.43 & 0.43 & 0.43 & 0.40 & 0.19 & $\ldots$ \\
\hline 98288 & 0.29 & 0.19 & -0.10 & 0.19 & $\ldots$ & $\ldots$ & -0.04 & 0.13 & 19.30 \\
\hline 98413 & 0.41 & 0.19 & 0.15 & 0.19 & $\ldots$ & $\ldots$ & 0.09 & 0.13 & $\ldots$ \\
\hline 98559 & 0.42 & 0.21 & 0.19 & 0.21 & $\ldots$ & $\ldots$ & 0.18 & 0.15 & $\ldots$ \\
\hline 98677 & 0.51 & 0.11 & 0.14 & 0.11 & 0.16 & 0.11 & 0.24 & 0.07 & 10.57 \\
\hline 98792 & 0.55 & 0.10 & -0.09 & 0.11 & -0.08 & 0.10 & 0.13 & 0.07 & $\ldots$ \\
\hline 98965 & 0.47 & 0.15 & 0.21 & 0.16 & $\ldots$ & $\ldots$ & 0.28 & 0.12 & $\ldots$ \\
\hline 99355 & 0.54 & 0.16 & 0.24 & 0.17 & 0.09 & 0.16 & 0.26 & 0.08 & $\ldots$ \\
\hline 99774 & 0.77 & 0.17 & -0.04 & 0.19 & $\ldots$ & $\ldots$ & 0.32 & 0.10 & $\ldots$ \\
\hline 99965 & 0.39 & 0.11 & 0.30 & 0.15 & 0.41 & 0.11 & 0.36 & 0.07 & $\ldots$ \\
\hline 100259 & 0.31 & 0.13 & 0.09 & 0.14 & $\ldots$ & $\ldots$ & -0.05 & 0.10 & 67.20 \\
\hline 101579 & 0.40 & 0.17 & 0.20 & 0.18 & $\ldots$ & $\ldots$ & 0.08 & 0.09 & $\ldots$ \\
\hline 101997 & 0.43 & 0.10 & 0.10 & 0.11 & 0.14 & 0.11 & $\ldots$ & $\ldots$ & $\ldots$ \\
\hline 102290 & 0.36 & 0.19 & 0.17 & 0.20 & $\ldots$ & $\ldots$ & 0.34 & 0.13 & 92.35 \\
\hline 102521 & 0.30 & 0.12 & 0.09 & 0.14 & -0.03 & 0.12 & 0.16 & 0.08 & 41.60 \\
\hline 103611 & 0.37 & 0.18 & 0.15 & 0.19 & $\ldots$ & $\ldots$ & 0.29 & 0.12 & 32.50 \\
\hline 103895 & 0.65 & 0.18 & 0.11 & 0.19 & $\ldots$ & $\ldots$ & 0.25 & 0.12 & $\ldots$ \\
\hline 103896 & 0.52 & 0.19 & 0.29 & 0.19 & $\ldots$ & $\ldots$ & 0.35 & 0.13 & $\ldots$ \\
\hline 104375 & 0.35 & 0.16 & -0.01 & 0.16 & -0.04 & 0.16 & 0.18 & 0.07 & $\ldots$ \\
\hline 104733 & 0.41 & 0.11 & 0.13 & 0.11 & 0.15 & 0.11 & 0.26 & 0.06 & 20.90 \\
\hline 106231 & 0.83 & 0.21 & -0.15 & 0.24 & $\ldots$ & $\ldots$ & -0.02 & 0.09 & $\ldots$ \\
\hline 106949 & 0.56 & 0.15 & 0.02 & 0.16 & $\ldots$ & $\ldots$ & 0.21 & 0.13 & $\ldots$ \\
\hline 107038 & 0.30 & 0.11 & 0.13 & 0.13 & 0.07 & 0.11 & 0.14 & 0.07 & 7.36 \\
\hline 107700 & 0.30 & 0.16 & 0.03 & 0.17 & -0.03 & 0.17 & 0.07 & 0.09 & $\ldots$ \\
\hline 108774 & 0.25 & 0.12 & 0.03 & 0.14 & -0.00 & 0.12 & 0.26 & 0.08 & 81.60 \\
\hline 108947 & 0.35 & 0.17 & 0.16 & 0.20 & $\ldots$ & $\ldots$ & 0.16 & 0.09 & $\ldots$ \\
\hline 109310 & 0.30 & 0.15 & 0.06 & 0.15 & $\ldots$ & $\ldots$ & 0.36 & 0.13 & 48.95 \\
\hline 110508 & 0.23 & 0.11 & -0.01 & 0.12 & 0.02 & 0.11 & 0.22 & 0.08 & $\ldots$ \\
\hline 111883 & 0.37 & 0.16 & 0.04 & 0.17 & -0.04 & 0.17 & 0.14 & 0.09 & $\ldots$ \\
\hline 111888 & 0.55 & 0.16 & 0.26 & 0.17 & 0.17 & 0.16 & 0.11 & 0.07 & $\ldots$ \\
\hline 111977 & 0.44 & 0.11 & -0.07 & 0.11 & -0.05 & 0.11 & 0.11 & 0.06 & $\ldots$ \\
\hline 112245 & 0.30 & 0.11 & 0.03 & 0.11 & 0.04 & 0.11 & 0.11 & 0.07 & $\ldots$ \\
\hline 112870 & 0.77 & 0.11 & 0.09 & 0.16 & 0.12 & 0.12 & 0.40 & 0.08 & $\ldots$ \\
\hline 113231 & 0.41 & 0.14 & -0.02 & 0.14 & $\ldots$ & $\ldots$ & 0.08 & 0.11 & 13.05 \\
\hline 113884 & 0.55 & 0.16 & 0.19 & 0.16 & $\ldots$ & $\ldots$ & 0.24 & 0.13 & 21.17 \\
\hline 113989 & 0.42 & 0.11 & -0.13 & 0.12 & -0.11 & 0.11 & -0.08 & 0.06 & $\ldots$ \\
\hline 114340 & 0.33 & 0.20 & 0.17 & 0.20 & $\ldots$ & $\ldots$ & 0.30 & 0.14 & 33.60 \\
\hline 114385 & 0.38 & 0.16 & 0.27 & 0.18 & $\ldots$ & $\ldots$ & 0.56 & 0.13 & 105.80 \\
\hline 115194 & 0.79 & 0.17 & 0.11 & 0.18 & $\ldots$ & $\ldots$ & 0.20 & 0.09 & $\ldots$ \\
\hline 115411 & 0.63 & 0.18 & 0.19 & 0.18 & $\ldots$ & $\ldots$ & -0.03 & 0.12 & $\ldots$ \\
\hline 115445 & 0.35 & 0.16 & 0.10 & 0.18 & -0.03 & 0.16 & 0.16 & 0.07 & $\ldots$ \\
\hline 116005 & 0.38 & 0.11 & 0.20 & 0.12 & 0.10 & 0.11 & 0.05 & 0.08 & $\ldots$ \\
\hline 116410 & 0.32 & 0.19 & -0.10 & 0.19 & $\ldots$ & $\ldots$ & 0.17 & 0.12 & $\ldots$ \\
\hline 118207 & 0.41 & 0.12 & 0.03 & 0.12 & 0.10 & 0.12 & 0.34 & 0.09 & 18.60 \\
\hline 118251 & 0.32 & 0.12 & -0.17 & 0.12 & -0.14 & 0.12 & 0.16 & 0.08 & $\ldots$ \\
\hline 118278 & 0.25 & 0.11 & 0.02 & 0.12 & 0.03 & 0.11 & 0.08 & 0.09 & $\ldots$ \\
\hline
\end{tabular}

$(-0.7 \leqslant[\mathrm{Fe} / \mathrm{H}] \leqslant-0.4)$ are relatively free from the sample bias, from which we found $\sigma\left(\Delta M_{V}\right)=0.11 \mathrm{mag}$ for the rms dispersion of these stars with highly accurate parallaxes $\left(\sigma_{\pi} / \pi \leqslant 0.03\right)$. On the other hand, the expected size of error in $\Delta M_{V}$ for individual stars is $0.16 \mathrm{mag}$ from $2 \%$ errors in the photometry, $\sim 2 \%$ error in parallax, and 0.067 dex error in metallicity (see above), which approximately matches the measured dispersion.
Figure 10 shows the same $\delta M_{V}$ versus $[\mathrm{Fe} / \mathrm{H}]$ diagrams as in the bottom panel of Figure 9, but in three different color bins: $0.6 \leqslant B-V<0.7 \quad$ (top), $0.7 \leqslant B-V<0.8 \quad$ (middle), and $0.8 \leqslant B-V \leqslant 1.0$ (bottom). The minimum $\delta M_{V}$ of stars in $0.8 \leqslant B-V \leqslant 1.0$ is slightly larger than those for bluer stars. As described in Section 2.1, this is because our colormagnitude selection, which was made using the Pleiades' MS, is not exactly parallel to the Hyades' MS (see the top lefthand 

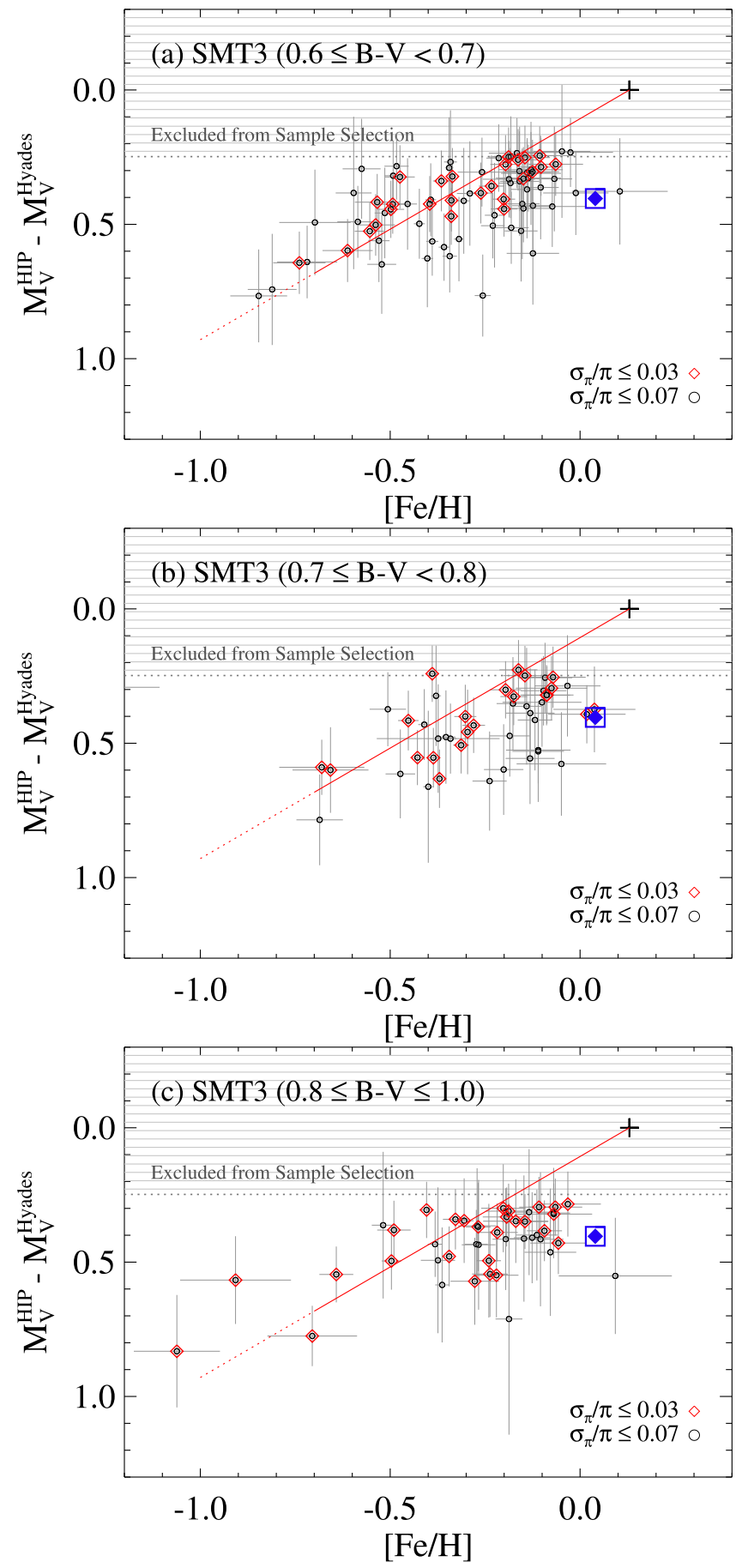

Figure 10. Same as in the bottom panel of Figure 9, but displaying KPNO samples in different color ranges: (a) $0.6 \leqslant B-V<0.7$, (b) $0.7 \leqslant B-V<0.8$, and (c) $0.8 \leqslant B-V \leqslant 1.0$.

panel in Figure 1). The Pleiades members progressively become fainter than older stars with the same metallicity or those predicted from standard stellar models, which is most likely due to stellar activities. The effect is mild at $0.9 \lesssim B-V \lesssim 1.0$, but is not convincingly observed for stars with $B-V<0.9$ (see Figure 20 in An et al. 2007b). We limited our sample to $B-V \leqslant 1$ because such activity-related change of stellar colors and magnitudes becomes severe for redder stars (Stauffer et al. 2003; An et al. 2007b). Other than a slight

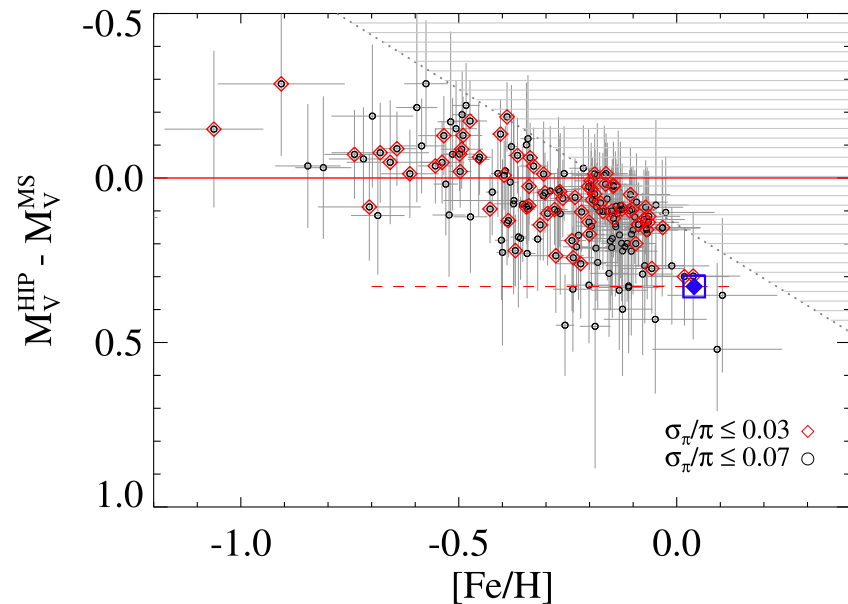

Figure 11. Same as in the bottom panel of Figure 9, but displaying $\Delta M_{V}$ or a difference between a MS-fitting distance modulus and that from the Hipparcos parallax.

difference in the minimum $\delta M_{V}$, our sample stars in Figure 10 behave almost independently of color ranges.

While most of the KPNO sample stars have Hipparcos parallaxes that are consistent with MS-fitting distances, a few stars have shorter Hipparcos distances. In the top panel of Figure 9, there is one such star found from the MOOG analysis (HIP 99965). In the bottom panel, which shows the same $\delta M_{V}$ with SMT3 metallicities, none of the stars with accurate parallaxes $\left(\sigma_{\pi} / \pi \leqslant 0.03\right.$; red diamond) have a larger magnitude excess than the Pleiades $\left(\Delta M_{V}=0.33\right.$; red dashed line). For the extended sample with $\sigma_{\pi} / \pi \leqslant 0.07$, a total of nine stars exhibit larger $\Delta M_{V}$ than the Pleiades, which account for $\sim 5 \%$ of the entire KPNO sample. These are HIP 5313, HIP 10276, HIP 73138, HIP 73845, HIP 74126, HIP 75446, HIP 78336, HIP 81831, and HIP 97668. The $\Delta M_{V}$ estimates of our sample stars, including those with the largest magnitude excess, are listed in the fourth and fifth columns in Table 7 . The errors in $\Delta M_{V}$ are larger than those of $\delta M_{V}$ due to an additional contribution from spectroscopic metallicity errors. Figure 11 shows $\Delta M_{V}$ of stars as a function of the SMT3 metallicity. The symbols are the same as in the bottom panel of Figure 9. The statistical significance of these nine stars is less $(\sim 2 \sigma)$ than that of the Pleiades, because individual stars have larger errors in $\delta M_{V}$ from photometry, metallicity, and parallax than in the case of a cluster where a large number of stars can be used together to increase an internal precision of the measurements.

Because stars with large $\Delta M_{V}$ all have relatively large parallax errors, it is unclear whether the large magnitude excess was induced by random parallax measurement errors or by a hidden systematic error in the Hipparcos parallax, which was originally suggested by Pinsonneault et al. (1998) to explain the short Pleiades distance. We also analyzed the spectra of the above nine stars with the largest magnitude excess using MOOG (Table 3); these stars were not originally included in our MOOG analysis due to their large parallax errors. With MOOG metallicities, six out of the nine stars still had larger $\Delta M_{V}$ than the Pleiades. Their $\Delta M_{V}$ estimates are shown in the sixth and seventh columns in Table 7, along with those for all stars analyzed using MOOG.

In addition to $B-V$ colors, we repeated the above experiment with $V-K_{s}$ colors using the MS of the Hyades in the $V-K_{s}$ versus $V$ CMD to compute $\delta M_{V}$ of individual stars. 

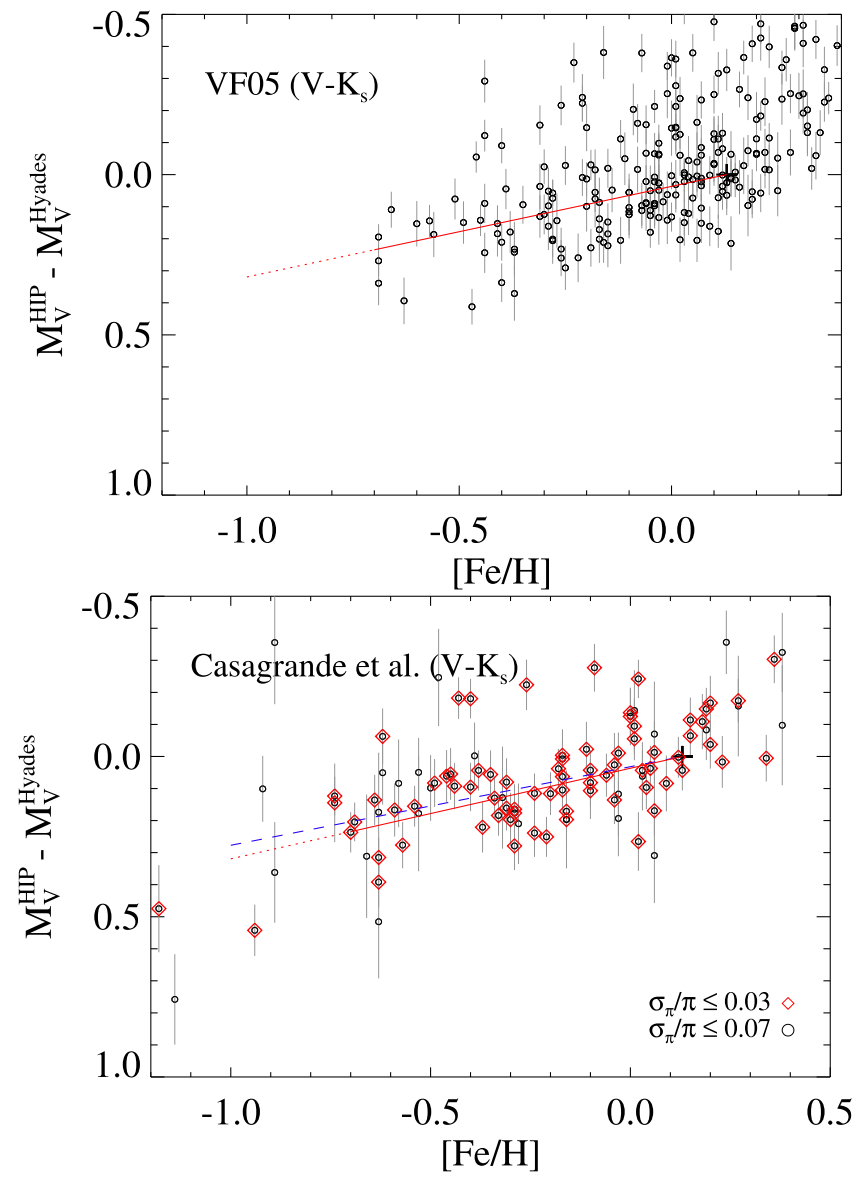

Figure 12. Same as in Figure 8, but in $V-K_{s}$ colors.

These $\delta M_{V}$ estimates are listed in the eighth and ninth columns in Table 7, which are not equal to $\delta M_{V}$ from $B-V$ because of a different color $-T_{\text {eff }}$ relation in $V-K_{s}$. The $V-K_{s}$ is also less sensitive to metallicity than $B-V$, while being more sensitive to unresolved binaries, giving an independent look at the distribution of stars in the $\delta M_{V}$ versus $[\mathrm{Fe} / \mathrm{H}]$ diagram. In addition, photometry from a uniform all sky survey like 2MASS can provide more reliable estimates in $\delta M_{V}$.

As in $B-V$, we derived a mean empirical metallicity sensitivity in $V-K_{s}$ using the same sample stars in VF05 with good parallaxes $\left(\sigma_{\pi} / \pi \leqslant 0.03\right)$. The result is shown in the top panel of Figure 12, where we used and displayed only those in $1.6 \leqslant V-K_{s} \leqslant 3.0$ to avoid contamination by turn-off stars. Stars with $-1.0 \leqslant[\mathrm{Fe} / \mathrm{H}] \leqslant-0.1$ and $\delta M_{V} \geqslant-0.5$ were used in the linear regression for the metallicity sensitivity function in $V-K_{s}$ (red solid line). The slope of this line is -0.28 mag dex $^{-1}$, which is significantly shallower than that from $B-V$ (Figure 8). The lower panel in Figure 12 also shows a metallicity sensitivity function in $V-K_{s}$ (blue line) as derived from an independent set of stars $\left(1.6 \leqslant V-K_{s} \leqslant 3.0\right)$ in Casagrande et al. (2010) with good parallaxes $\left(\sigma_{\pi} / \pi \leqslant 0.03\right)$, of which slope $\left(-0.25 \mathrm{mag} \mathrm{dex}^{-1}\right)$ is almost identical to the one from the top panel (red line).

Our KPNO sample stars are shown in Figure 13, where $\delta M_{V}$ was derived from $V-K_{s}$ CMDs. As in Figure 9, MOOG metallicities are used in the top panel, and $[\mathrm{Fe} / \mathrm{H}]$ from SMT3 are used in the bottom panel. Stars with highly accurate parallaxes $\left(\sigma_{\pi} / \pi \leqslant 0.03\right)$ are shown in the top panel and indicated by red diamond points in the bottom panel. The red
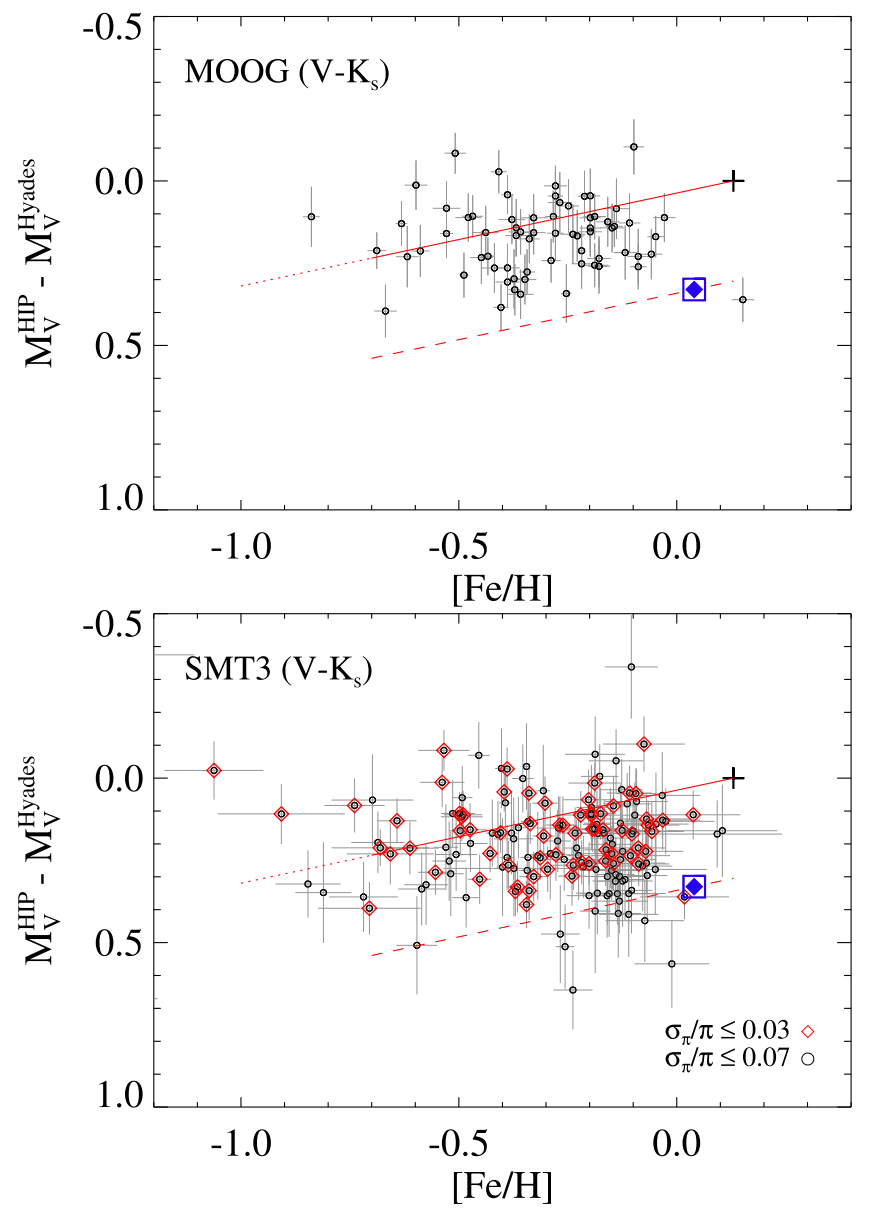

Figure 13. Same as in Figure 9, but in $V-K_{s}$ colors.

solid line is the mean metallicity sensitivity function from the top panel in Figure 12. Most of our sample stars follow this trend, if one takes into account the fact that our sample selection was biased against stars with small (negative) $\delta M_{V}$. The $\delta M_{V}$ of the Pleiades, as determined from the Hyades' MS, is $\delta M_{V}=0.33$ (or $\Delta M_{V}=0.30$ ), and is shown as a blue diamond symbol.

In total, nine stars were identified as having larger $\Delta M_{V}$ than the Pleiades (red dashed line): HIP 5313, HIP 56092, HIP 71720, HIP 75446, HIP 77810, HIP 81831, HIP 97668, HIP 99965, and HIP 114385. However, only about half (HIP 5313, HIP 75446, HIP 81831, and HIP 97668) show as large $\Delta M_{V}$ as the Pleiades in $B-V$ (Table 7), suggesting that photometric errors would have made a significant contribution to an error in $\Delta M_{V}$. We also analyzed these stars using MOOG (Table 3), because they were not originally included in our MOOG analysis (except HIP 99965) due to large parallax errors $\left(\sigma_{\pi} / \pi>0.03\right)$. Nevertheless, we found that the difference in metallicity between the two approaches is small. Its impact on $\Delta M_{V}$ is further reduced by the relatively weak metallicity dependence in $V-K_{s}\left(-0.28\right.$ mag dex $\left.^{-1}\right)$, resulting in a negligible difference in $\Delta M_{V}$.

Figure 14 shows the positions of the KPNO samples in equatorial coordinates, which by design is randomly distributed at $\delta>-30^{\circ}$ in our observing programs. The red bull's-eyes are the positions of the nine stars with the largest magnitude excess in $B-V$ CMDs, and red triangles are those from $V-K_{s}$. In either case, these stars are not spatially correlated with one 


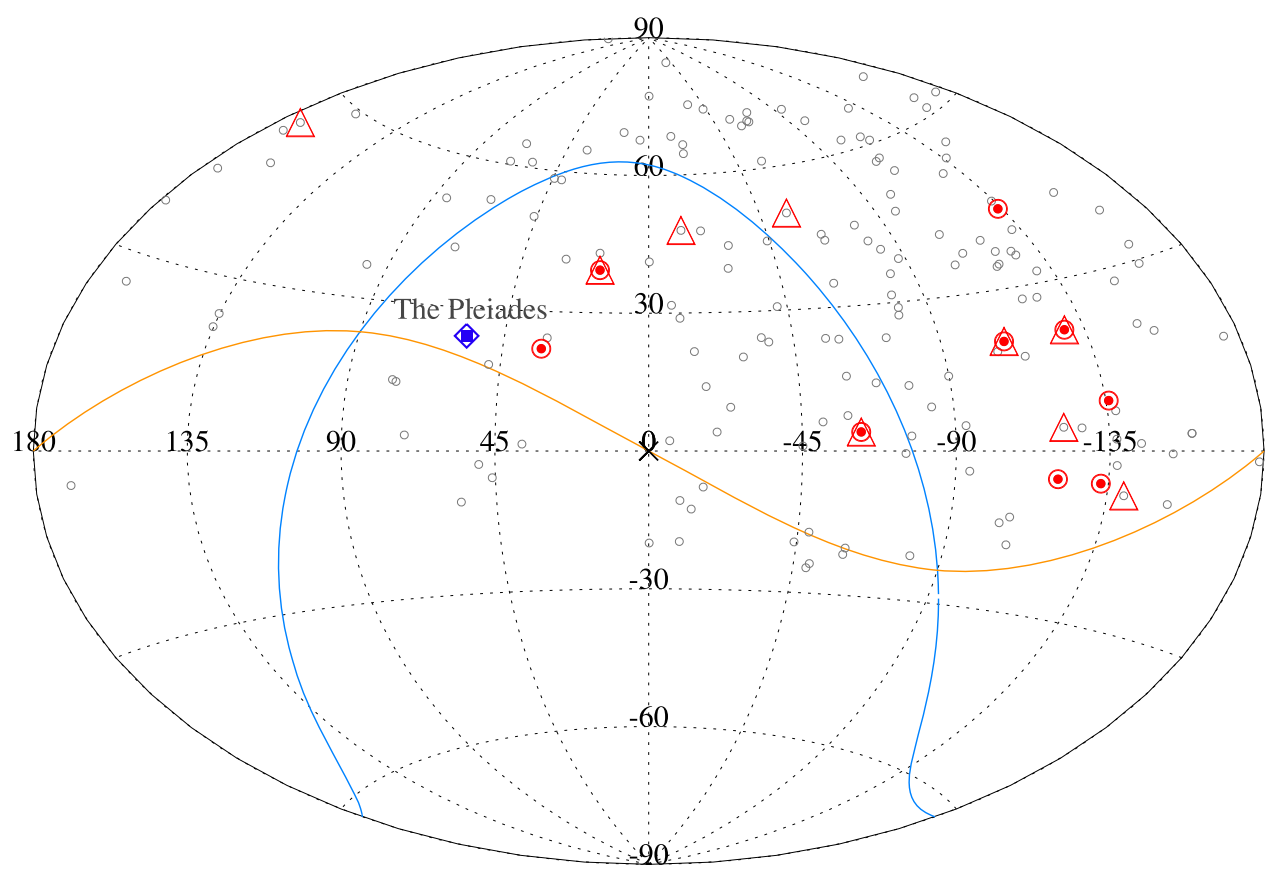

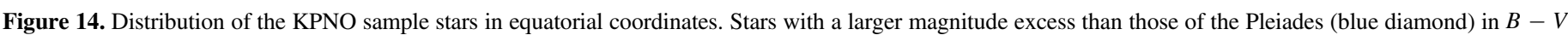

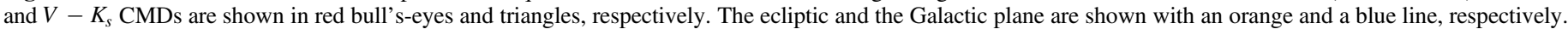

another, nor do they show a spatial correlation with the location of the Pleiades (blue diamond point). Also, they are not associated with any of the fundamental great circles, such as the ecliptic (orange line) or the Galactic plane (blue line).

Our result shows that there are a small fraction of stars with sufficiently large $\Delta M_{V}$, but their statistical significance is only marginal (a $2 \sigma$ level). On the other hand, we failed to unambiguously identify stars with a large magnitude excess like the Pleiades, independently of a spectroscopic analysis technique and a color index employed, among those having good parallaxes $\left(\sigma_{\pi} / \pi<0.03\right)$. This may suggest that stars with the Pleiades-like phenomenon are rare, at least among nearby field stars with good parallax measurements. To confidently identify stars with (still unknown) systematic errors in parallax, it would be necessary to further shrink the size of errors in photometry and metallicity.

\subsection{Young Age and Stellar Activity}

Young stars often exhibit chromospheric activities with large stellar spots, which are thought to be related to their large angular momenta. All together, one can naively expect either of these effects to somehow modify stellar energy distributions, making their observed broadband colors or magnitudes deviate from those of older stars. Such color anomalies have already been observed from late K-type dwarfs in the Pleiades (Stauffer et al. 2003), but our KPNO sample covers spectral types earlier than $\sim \mathrm{K} 2$, which helps to avoid issues on the potential modifications of colors and magnitudes by stellar activity and/ or young age. In the following, we focus on young and/or active stars on the $\delta M_{V}$ versus $[\mathrm{Fe} / \mathrm{H}]$ diagram, and see if these stars have systematically shorter Hipparcos distances than older stars. This will directly test a hypothesis that the longer Pleiades distance from MS fitting is due to yet unknown physics of young/active stars in the cluster (van Leeuwen 1999).

\subsubsection{Lithium Absorptions}

The color-magnitude selection of our sample is biased against the most metal-rich stars in the disk, and therefore probably does not favor a selection of young $(\lesssim 200$ Myr $)$ stars in the solar neighborhood. On the other hand, very young stars $(\lesssim 30 \mathrm{Myr})$ are also difficult to detect in our survey because low-mass pre-MS stars are brighter than MS at a given color. We measured EWs of lithium absorption at $6707.70 \AA$ for our KPNO stars, and found that about $25 \%$ of the entire KPNO sample $(N=42)$ shows an EW of $\mathrm{Li}$ larger than $5 \mathrm{m \AA}$. However, a majority show weak absorptions, suggesting that most of our sample stars, including those with non-detections, are relatively old. Among these, however, a small number of our KPNO spectra revealed a relatively strong $\mathrm{Li}$ absorption, implying young ages of these objects. The EWs of stars having $W(\mathrm{Li})>5 \mathrm{~mA}$ are listed in the last column of Table 7.

While the Li line strength becomes weaker as a star gets older, an EW of lithium is also strongly dependent on stellar colors (or mass) by the different depths of the outer convective cells and by different amounts of angular momentum. This is shown in the top panel of Figure 15, which displays lithium EWs of MS stars in three open clusters as a function $B-V$ (see Soderblom 2010): the Pleiades (blue open circle; Butler et al. 1987; Soderblom et al. 1993; Garcia Lopez et al. 1994; Jones et al. 1996; Jeffries 1999), the Hyades (black open box; Soderblom et al. 1990, 1995; Thorburn et al. 1993), and M67 (open diamond; Hobbs \& Pilachowski 1986; Spite et al. 1987; Garcia Lopez et al. 1988; Pasquini et al. 1997; Jones et al. 1999; Randich et al. 2002) at the age of $100 \mathrm{Myr}$, $550 \mathrm{Myr}$, and $4 \mathrm{Gyr}$, respectively. On top of these, our KPNO sample stars are marked by red closed circles. Only a few stars in our sample fall into the range of $\mathrm{Li}$ EWs covered by the Pleiades members, which is not surprising given that our sample selection on a CMD was not designed to find the youngest stars in the solar neighborhood. Due to a relatively small number of such stars, we selected the young stars as those 

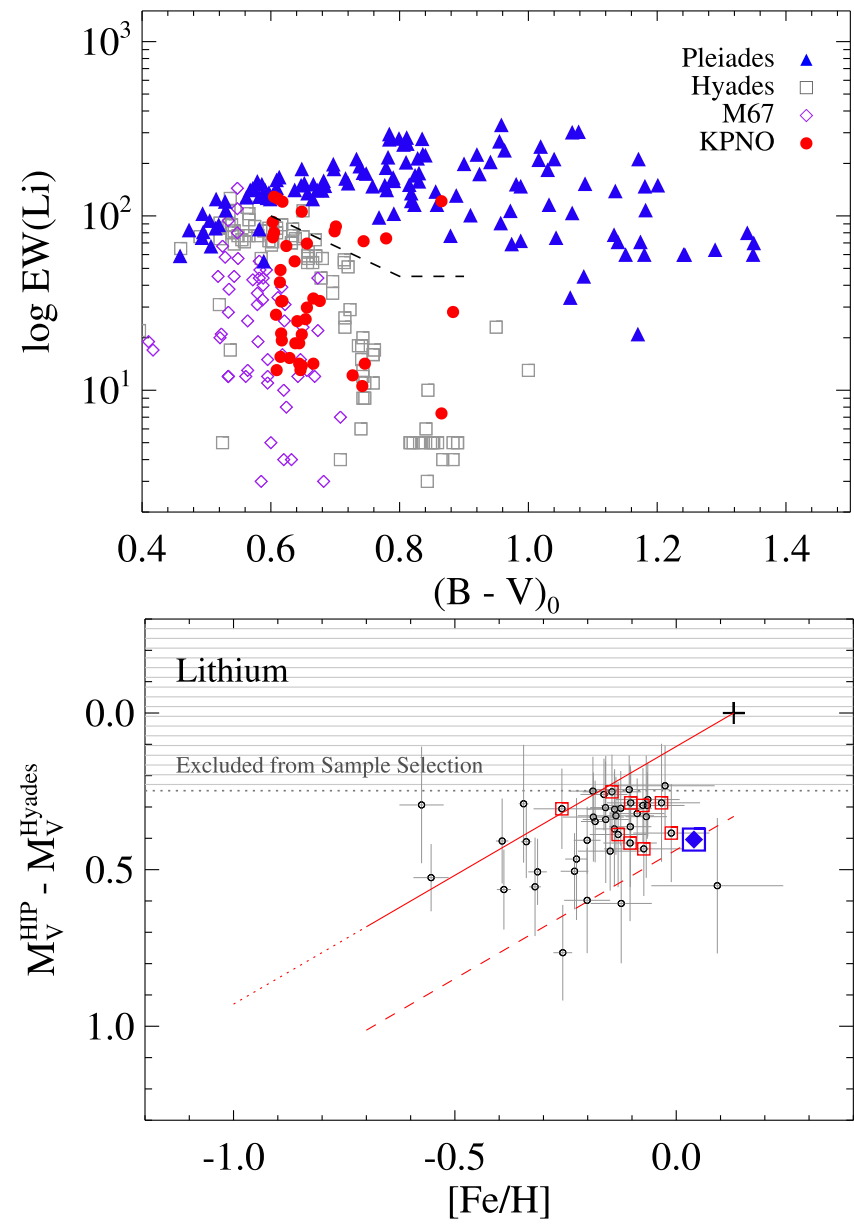

Figure 15. Top: EWs of lithium $6707 \AA$ for the KPNO sample (red filled circles). EWs from open clusters are shown for the Pleiades (100 Myr; blue filled triangle), the Hyades (550 Myr; gray open box), and M67 (4 Gyr; open diamond). Our selection of young stars is indicated by a dashed line. Bottom: same as in the bottom panel of Figure 9, but displaying stars with lithium $6707 \AA$ absorptions ( $\geqslant 5 \mathrm{~m} \AA$ ). Stars with EWs larger than the dashed line in the top panel are shown in red squares.

having larger Li EWs than those found in the Hyades, as shown by a dashed line. This selection includes nine stars (HIP 21276, HIP 60074, HIP 63322, HIP 72703, HIP 76674, HIP 77810, HIP 82388, HIP 108774, and HIP 114385). The star with an exceptionally large $\mathrm{Li} \mathrm{EW}[W(\mathrm{Li}) \approx 120 \mathrm{m \AA}]$ at $B-V=0.87$ is HIP 63322 .

The bottom panel in Figure 15 shows the same $\delta M_{V}$ versus $[\mathrm{Fe} / \mathrm{H}]$ diagram of stars as in the bottom panel of Figure 9 with SMT3 metallicities, but displaying stars with a lithium EW larger than $5 \mathrm{~mA}$. The red boxed points are the young stars selected previously as having largest Li EWs. As seen in this panel, the distribution of stars with lithium absorptions is not dissimilar to those seen from older stars (Figure 9). Among these, three stars (HIP 5313, HIP 74126, and HIP 78336) exhibit a larger magnitude excess than the Pleiades $(\Delta M \geqslant 0.33)$, but do not show a sufficiently strong Li absorption. More importantly, none of the above selected young stars exhibit larger $\Delta M_{V}$ than the Pleiades. Even if the sample is further restricted to the five stars with $W(\mathrm{Li})>100 \mathrm{m \AA}$ that have almost identical properties to those in the Pleiades, their $\Delta M_{V}$ values show no systematic offset with respect to the empirical metallicity sensitivity function (red solid line). On the other hand, if the Pleiades' distance is short because of the young age of its stars, we would expect that stars with a similar Li strength to the Pleiades members should have systematically large $\Delta M_{V}$. Therefore, our result in Figure 15 suggests that the short Pleiades' distance cannot be condemned to somehow modified color-magnitude relations for young stars in the cluster, and that MS-fitting distances for young stars, with spectral types earlier than $\sim \mathrm{K} 2$, are sufficiently close to those derived using colormagnitude relations for older stars.

Figure 16 shows the CMDs of the Pleiades (Stauffer et al. 2007; Kamai et al. 2014) assuming the short (left panel) and the long (right panel) distance scale of the cluster, respectively. The most recent Hipparcos distance in van Leeuwen (2009) is used in the left panel. For the long-distance scale, we used a weighted average distance $(m-M)_{0}=5.647 \pm 0.013$ $(134.7 \mathrm{pc})$ from a number of geometric measurements (trigonometric parallaxes and binary solutions) listed in An et al. (2007b) and the VLBI measurement (Melis et al. 2014). We assumed $E(B-V)=0.032$ for the Pleiades (An et al. 2007b) with colordependent reddening and extinction laws (An et al. 2007a). The red open circles are our KPNO stars selected as having strong Li absorptions. For an apple-to-apple comparison with the Pleiades stars, we corrected their $V$-band magnitudes for a metallicity difference from the Pleiades $([\mathrm{Fe} / \mathrm{H}]=+0.04)$ using the empirical metallicity sensitivity function (i.e., red line in Figure 8). As these stars have lower metallicities than the Pleiades, they become brighter with these corrections.

The comparison with the Pleiades' CMDs in Figure 16 shows that Li-rich young stars are consistently brighter than the MS of the Pleiades by $\Delta M_{V} \approx 0.25$ when the Hipparcos distance is assumed (left hand panel). ${ }^{16}$ On the other hand, the long-distance scale of the cluster leads to an excellent match of young field stars with the observed MS of the Pleiades. An absolute metallicity scale for our sample could be in error. However, $\Delta M_{V}=0.08 \mathrm{mag}$ is expected if we have consistently overestimated our metallicities (or equivalently underestimated the Pleiades' metallicity) by $\Delta[\mathrm{Fe} / \mathrm{H}]=0.1$, which is far smaller than what is required to explain the $\Delta M_{V} \approx 0.25$ difference with the Pleiades' MS in the left panel.

In fact, most of these Li-rich stars are fainter than the mean MS relation at a given metallicity (with respect to the mean empirical metallicity sensitivity function) because of the sample selection bias as delineated by a shaded area in the bottom panel of Figure 15. Therefore, the red circles in Figure 16 represent approximately half of an Li-rich population in a given metallicity range, and constitute only the lower half of the brightness distribution. Nonetheless, missing Li-rich stars would be found above the red circles in the $B-V$ CMD (Figure 16), and would make the agreement with the Pleiades' MS even worse if the Hipparcos parallax is assumed for the cluster's distance. Figure 16 simply restates our conclusion that the Pleiades distance in van Leeuwen (2009) is too short and cannot be explained with the young age of cluster members.

\subsubsection{Stellar Activity Indices}

One of the most frequently used activity indicators is the $R_{\mathrm{HK}}^{\prime}$ index, which measures the chromospheric emission line strength of $\mathrm{Ca}$ II $\mathrm{H}$ and $\mathrm{K}$ at $3933.7 \AA$ and $3968.5 \AA$ in the central part of its broad absorption profile, as normalized by

\footnotetext{
16 This does not contradict our sample selection (Section 2.1), in which we selected stars that are near or below the MS of the Pleiades assuming the van Leeuwen (2009) distance, because of the metallicity corrections described above.
} 

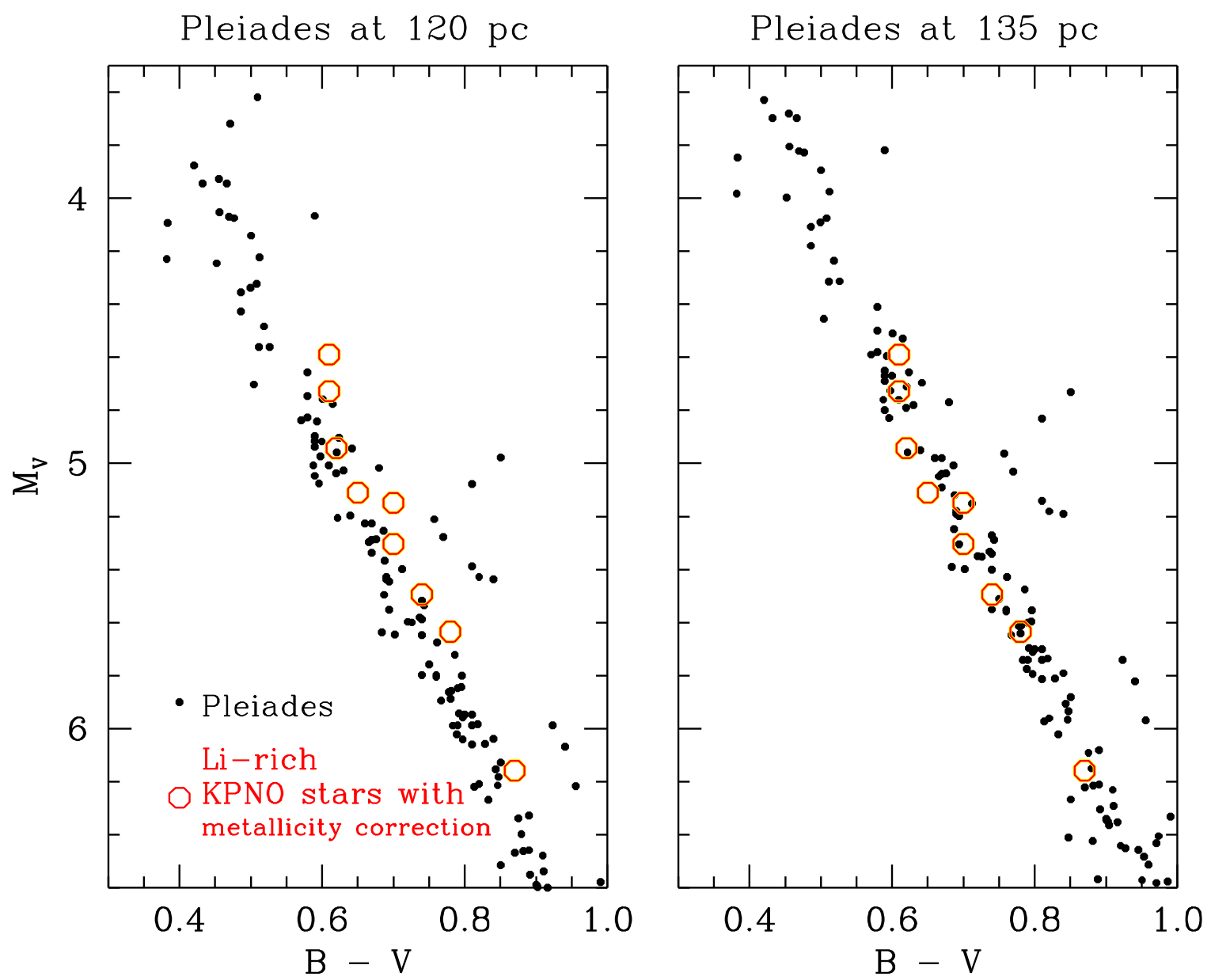

Figure 16. CMDs of the Pleiades (black points) assuming the Hipparcos parallax measurement in van Leeuwen (2009; left panel) and the mean distance of the cluster from a number of geometric distance measurements, except that from Hipparcos (right panel). The $E(B-V)=0.032$ is assumed for hypothetical zero-color stars in the cluster with color-dependent reddening laws. The red open circles are Li-rich KPNO stars (those above the dashed line in Figure 15) with $V$-band magnitudes corrected for a metallicity difference from the Pleiades (see text).

photospheric continuum emissions (see Noyes et al. 1984). The $R_{\mathrm{HK}}^{\prime}$ is a function of $T_{\mathrm{eff}}$ or colors, and is known to decrease with stellar ages (see Soderblom 2010). This is shown in the top panel of Figure 17, where $R_{\mathrm{HK}}^{\prime}$ measurements from individual stars in three fiducial open clusters (Pleiades, the Hyades, and M67; Mamajek \& Hillenbrand 2008) are displayed (see also Soderblom 2010). The $R_{\mathrm{HK}}^{\prime}$ measurements from the Pleiades show a large scatter, indicating a star-to-star variation in chromospheric emissions, and a separation from those in the Hyades is not clearcut. Nevertheless, the cluster observations clearly suggest a systematic change of $R_{\mathrm{HK}}^{\prime}$ with age.

In the top panel of Figure 17, our KPNO sample stars are indicated by red closed circles on top of the cluster observations. We took a large compilation of S-index measurements in the literature (Pace 2013, see references therein). In total, 93 stars in our sample have valid S-index measurements. Following Pace (2013), we took the average of the minimum and maximum S-index values for each star, whenever there are repeat measurements, as a proxy for a timeaveraged chromospheric activity level. We adopted a procedure in Noyes et al. (1984) to convert $\mathrm{S}$ index into $R_{\mathrm{HK}}^{\prime}$.

Evidently, most stars in Figure 17 have similar $R_{\mathrm{HK}}^{\prime}$ values with those in M67, suggesting the old ages of these stars. On the other hand, there are approximately a dozen stars with $R_{\mathrm{HK}}^{\prime}$ values that are comparable to those in the Pleiades. We took $\log R_{\mathrm{HK}}^{\prime}=-4.5$ (dashed line) to select 11 likely young stars in our sample: HIP 4907, HIP 21276, HIP 60074, HIP 63322, HIP 63636, HIP 76674, HIP 77810, HIP 82388, HIP 106231, HIP 108774, and HIP 111888. Most of the Li-rich stars were also selected as having a strong chromospheric activity. The remaining Li-rich stars (HIP 72703 and HIP 114385) either do not have a $R_{\mathrm{HK}}^{\prime}$ measurement in the literature or have a value near $\log R_{\mathrm{HK}}^{\prime}=-4.5$. Nevertheless, our selection of active stars from $R_{\mathrm{HK}}^{\prime}$ includes many Hyades dwarfs, and the separation of young stars from older populations is not as clear as in Figure 15 based on a lithium absorption.

The bottom panel of Figure 17 displays stars with $R_{\mathrm{HK}}^{\prime}$ measurements on the $\delta M_{V}$ versus $[\mathrm{Fe} / \mathrm{H}]$ diagram with SMT3 metallicities. As expected, most of these stars are old and are found along the mean metallicity sensitivity function (red solid line). Meanwhile, the boxed points are stars with $\log R_{\mathrm{HK}}^{\prime}>-4.5$, and none of these chromospherically active stars show a larger magnitude excess than the Pleiades $\left(\Delta M_{V}>0.33\right)$. This result reiterates our conclusion based on Li-rich stars, that a young age of star does not significantly modify color-magnitude relations for MS dwarfs.

In addition to $R_{\mathrm{HK}}^{\prime}$ index, we collected the X-ray luminosities of our sample stars from the NASA Exoplanet archive (Ramirez et al. 2013) and looked into the properties of the stars selected based on X-ray luminosity. The X-ray luminosities show tight correlations with chromospheric activities, such 

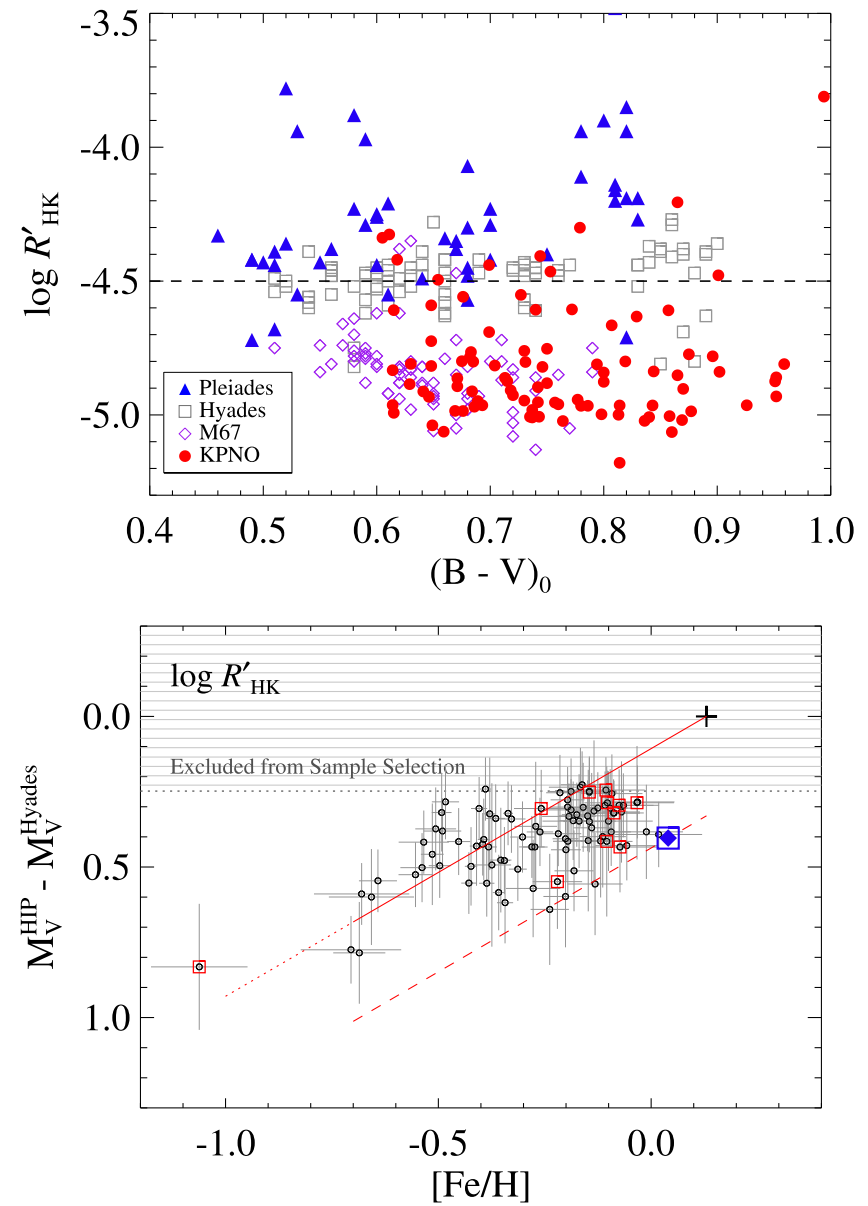

Figure 17. Top: $R_{\mathrm{HK}}^{\prime}$ values for the KPNO sample in comparison with cluster measurements: the Pleiades (100 Myr; blue filled triangle), the Hyades (550 Myr; gray open box), and M67 (4 Gyr; open diamond). The black dashed line represents our division of active/inactive stars based on $R_{\mathrm{HK}}^{\prime}$. Bottom: same as in the bottom panel of Figure 9, but displaying stars with $R_{\mathrm{HK}}^{\prime}$ values available in the literature. The red boxed points are stars with $\log R_{\mathrm{HK}}^{\prime}>-4.5$.

as $R_{\mathrm{HK}}^{\prime}$ (Mamajek \& Hillenbrand 2008), and can be used to trace young populations in the disk. However, measurements of X-ray luminosity are available only for 10 stars in our sample (Huensch et al. 1998; Hünsch et al. 1999; Schmitt \& Liefke 2004). Among these, only two stars (HIP 21276, HIP 106231) exhibit a higher X-ray luminosity than those in the Pleiades $\left(L_{X}=29.00 \mathrm{erg} \mathrm{s}^{-1}\right.$; Daniel et al. 2002). However, these stars have a significantly smaller magnitude excess than the Pleiades members. Although the number of stars with $\mathrm{X}$-ray luminosities in the literature is small, it is clear that active stars in our sample have the same photometric properties as those for older stars. We conclude that the stellar activity or young age of stars has little impact (if any) on a large magnitude excess.

\subsection{Empirical MS-fitting Distance to the Pleiades}

Given the lack of evidence on anomalous color-magnitude relations for young/active stars with $0.6 \leqslant B-V \leqslant 1.0$, a purely empirical MS-fitting distance to the Pleiades can be obtained using the observed MS of the Hyades and the empirical metallicity sensitivity function as derived from the Hipparcos dwarfs. We assumed $E(B-V)=0.032$ for the Pleiades (see above) and obtained $(m-M)_{0}=5.657 \pm 0.017$ and $5.669 \pm 0.024$ (statistical) in the $B-V$ and $V-K_{s}$ CMDs, respectively, using the photometry of the single MS stars of the cluster (An et al. 2007b) in $0.6 \leqslant B-V \leqslant 1.0$ and the corresponding range in $V-K_{s}$. We corrected the distance moduli for a metallicity difference between the Pleiades and the Hyades, based on the empirical metallicity sensitivity functions in each of the color indices (red lines in Figures 8 and 12), which results in $(m-M)_{0}=5.585 \pm 0.023$ and $5.644 \pm 0.025$ in the $B-V$ and $V-K_{s}$ CMDs, respectively, where the errors are a quadrature sum of errors in fitting and metallicity $\left(\sigma_{[\mathrm{Fe} / \mathrm{H}]}=0.02\right)$. The average distance modulus of the Pleiades from the two CMDs becomes $(m-M)_{0}=5.615 \pm 0.030$; the error represents half the difference in distance modulus. This purely empirical distance modulus is in perfect agreement with the mean geometric distance modulus of the cluster $\left[(m-M)_{0}=5.647 \pm 0.013\right]$, but is significantly longer than the Hipparcos-based distance $(m-M)_{0}=5.40 \pm 0.03$ (van Leeuwen 2009).

\section{SUMMARY}

The debate on the Pleiades distance has continued even after the new reduction of the Hipparcos parallaxes (van Leeuwen 2009), which predicts $\sim 0.3 \mathrm{mag}$ fainter magnitudes of the cluster stars than those expected from MS fitting or other independent distance determinations. In this study, we tested a hypothesis that the long photometric distance of the Pleiades is due to the anomalous colors or magnitudes of its cluster members, by searching for hypothesized sub-luminous field stars in the Hipparcos catalog. For comparison with the Hipparcos parallax, we derived accurate metallicities of 170 nearby G- and K-type field dwarfs based on high S/N, highresolution spectra, employing two independent spectral analysis techniques (MOOG and SMT). Our photometric distances based on these metallicities are purely empirical, being independent of any theoretical stellar isochrones, and rely on the observed MS of the Hyades and the metallicity dependence of colors, which was derived from the Hipparcos parallaxes and our metallicity measurements for a large number of field stars.

Among stars with highly accurate parallaxes $\left(\sigma_{\pi} / \pi \leqslant 0.03\right)$, we could identify only one star from a $B-V$ CMD with a larger magnitude excess $\left(\Delta M_{V}\right)$ than the Pleiades, or a shorter Hipparcos-based distance by $\Delta(m-M)_{0}=\Delta M_{V} \geqslant 0.33$, from the MOOG analysis. However, none of the stars in our sample have a larger magnitude excess than the Pleiades when the SMT metallicities are employed. For an extended sample with $\sigma_{\pi} / \pi \leqslant 0.07$, we identified nine stars with $\Delta M_{V} \geqslant 0.33$, but the differences in the distance modulus of these stars are only marginal. Furthermore, only six out of the nine stars remain to show a large magnitude excess with an independent analysis technique from MOOG. In addition to $B-V$, we repeated the above exercise with $V-K_{s}$ colors, and found that only three stars identified as having $\Delta M_{V} \geqslant 0.33$ from $B-V$ have larger magnitude excesses than the Pleiades in $V-K_{s}$. Therefore, their oddity may not be surprising at all, and can be understood from errors in photometry, metallicity, and parallax.

Although we could not identify stars with large magnitude excesses at a statistically significant level, we were able to reject the hypothesis that these outlying stars are mostly young or active stars. We selected young/active stars based on the $\mathrm{Li}$ $6707 \AA$ absorption, $R_{\mathrm{HK}}^{\prime}$, or X-ray luminosity, and found that the photometric distances of these young/active stars are not greatly different from the Hipparcos parallaxes. Although only 
a few stars in our sample may be as young as those in the Pleiades, none show larger differences in distance from Hipparcos than the Pleiades. While more young star samples can be used to better quantify the difference in distance, our result suggests that the short Pleiades distance is not at least directly related to the young age of the cluster.

The successful launch of Gaia (Perryman et al. 2001) has opened a new era in the studies of stars in the Milky Way Galaxy, which will deliver precise astrometric data for about one billion stars with more than two orders of magnitude improvement in parallax measurements than available in the past. It is hoped that Gaia will eventually help to resolve the Pleiades distance problem and will cast new light on hidden systematic errors in the Hipparcos parallax measurements. However, the Pleiades distance controversy has revealed a practical limit in the analysis of space-based astrometric data, which leads to demand for a careful check on the future parallax measurements. Our analysis technique based on accurate spectroscopic data can be utilized to assess the accuracy of parallax measurements by Gaia.

We thank an anonymous referee for various suggestions, which helped to improve the readability of the manuscript. We thank Courtney Epstein for her assistance in the observations. B.K. and D.A. acknowledge support provided by the National Research Foundation of Korea to the Center for Galaxy Evolution Research (No. 2010-0027910) and by Basic Science Research Program through the National Research Foundation of Korea (NRF) funded by the Ministry of Education (20100025122, 2015R1D1A1A09058700). This work has developed from a master's thesis conducted by B.K. under the supervision of D.A. at Ewha Womans University. J.R.S. gratefully acknowledges funding support from NASA Kepler grant NNX1AV62G. Y.S.L. acknowledges support provided by the National Research Foundation of Korea to the Center for Galaxy Evolution Research (No. 2010-0027910) and the Basic Science Research Program through the National Research Foundation of Korea (NRF) funded by the Ministry of Science, ICT \& Future Planning (NRF-2015R1C1A1A02036658). D.M.T. acknowledges support from award AST-1411685 from the National Science Foundation to The Ohio State University. This research has made use of the NASA Star and Exoplanet Database (NStED), which was operated until 2011 by the California Institute of Technology, under contract with the National Aeronautics and Space Administration. This research has made use of the SIMBAD database, operated at CDS, Strasbourg, France.

\section{REFERENCES}

An, D., Terndrup, D. M., \& Pinsonneault, M. H. 2007a, ApJ, 671, 1640 An, D., Terndrup, D. M., Pinsonneault, M. H., et al. 2007b, ApJ, 655, 233 An, D., Terndrup, D. M., Pinsonneault, M. H., \& Lee, J.-W. 2015, ApJ, 811, 46 Anders, E., \& Grevesse, N. 1989, GeCoA, 53, 197

Aumer, M., \& Binney, J. J. 2009, MNRAS, 397, 1286

Belikov, A. N., Hirte, S., Meusinger, H., Piskunov, A. E., \& Schilbach, E. 1998, A\&A, 332, 575

Bensby, T., Feltzing, S., \& Lundström, I. 2003, A\&A, 410, 527

Bensby, T., Feltzing, S., \& Oey, M. S. 2014, A\&A, 562, AA71

Boesgaard, A. M., Roper, B. W., \& Lum, M. G. 2013, ApJ, 775, 58

Butler, R. P., Marcy, G. W., Cohen, R. D., \& Duncan, D. K. 1987, ApJL, 319, L19

Casagrande, L., Ramírez, I., Meléndez, J., Bessell, M., \& Asplund, M. 2010, A\&A, 512, A54

Casagrande, L., Silva Aguirre, V., Schlesinger, K. J., et al. 2016, MNRAS, 455,987
Castelli, F., \& Kurucz, R. L. 2004, arXiv:astro-ph/0405087

Daniel, K. J., Linsky, J. L., \& Gagné, M. 2002, ApJ, 578, 486

de Bruijne, J. H. J., Hoogerwerf, R., \& de Zeeuw, P. T. 2001, A\&A, 367, 111 Garcia Lopez, R. J., Rebolo, R., \& Beckman, J. E. 1988, PASP, 100, 1489

Garcia Lopez, R. J., Rebolo, R., \& Martin, E. L. 1994, A\&A, 282, 518

Grevesse, N., \& Sauval, A. J. 1999, A\&A, 347, 348

Hobbs, L. M., \& Pilachowski, C. 1986, ApJL, 311, L37

Høg, E., Fabricius, C., Makarov, V. V., et al. 2000, A\&A, 355, L27

Huensch, M., Schmitt, J. H. M. M., \& Voges, W. 1998, A\&AS, 132, 155

Hünsch, M., Schmitt, J. H. M. M., Sterzik, M. F., \& Voges, W. 1999, A\&AS, 135,319

Jeffries, R. D. 1999, MNRAS, 309, 189

Jones, B. F., Fischer, D., \& Soderblom, D. R. 1999, AJ, 117, 330

Jones, B. F., Shetrone, M., Fischer, D., \& Soderblom, D. R. 1996, AJ, 112, 186 Kamai, B. L., Vrba, F. J., Stauffer, J. R., \& Stassun, K. G. 2014, AJ, 148, 30 Lee, Y. S., Beers, T. C., Sivarani, T., et al. 2008a, AJ, 136, 2022

Lee, Y. S., Beers, T. C., Sivarani, T., et al. 2008b, AJ, 136, 2050

Makarov, V. V. 2002, AJ, 124, 3299

Makarov, V. V. 2003, AJ, 126, 2408

Mamajek, E. E., \& Hillenbrand, L. A. 2008, ApJ, 687, 1264

Mamajek, E. E., Meyer, M. R., \& Liebert, J. 2002, AJ, 124, 1670

Mamajek, E. E., Meyer, M. R., \& Liebert, J. 2006, AJ, 131, 2360

Markwardt, C. B. 2009, adass XVIII, 411, 251

Melis, C., Reid, M. J., Mioduszewski, A. J., Stauffer, J. R., \& Bower, G. C. 2014, Sci, 345, 1029

Munari, U., Dallaporta, S., Siviero, A., et al. 2004, A\&A, 418, L31

Narayanan, V. K., \& Gould, A. 1999, ApJ, 523, 328

Noyes, R. W., Hartmann, L. W., Baliunas, S. L., Duncan, D. K., \& Vaughan, A. H. 1984, ApJ, 279, 763

Pace, G. 2013, A\&A, 551, L8

Pan, X., Shao, M., \& Kulkarni, S. R. 2004, Natur, 427, 326

Pasquini, L., Randich, S., \& Pallavicini, R. 1997, A\&A, 325, 535

Paulson, D. B., Sneden, C., \& Cochran, W. D. 2003, AJ, 125, 3185

Percival, S. M., Salaris, M., \& Kilkenny, D. 2003, A\&A, 400, 541

Perryman, M. A. C., Brown, A. G. A., Lebreton, Y., et al. 1998, A\&A, 331, 81 Perryman, M. A. C., de Boer, K. S., Gilmore, G., et al. 2001, A\&A, 369, 339 Perryman, M. A. C., Lindegren, L., Kovalevsky, J., et al. 1997, A\&A, 323, L49

Pinsonneault, M. H., Stauffer, J., Soderblom, D. R., King, J. R., \& Hanson, R. B. 1998, ApJ, 504, 170

Pinsonneault, M. H., Terndrup, D. M., Hanson, R. B., \& Stauffer, J. R. 2004, ApJ, 600, 946

Portinari, L., Casagrande, L., \& Flynn, C. 2010, MNRAS, 406, 1570

Pourbaix, D., Tokovinin, A. A., Batten, A. H., et al. 2004, A\&A, 424, 727

Ramirez, S., Akeson, R. L., Ciardi, D., et al. 2013, BAAS, 221, 334.01

Randich, S., Primas, F., Pasquini, L., \& Pallavicini, R. 2002, A\&A, 387, 222

Schmitt, J. H. M. M., \& Liefke, C. 2004, A\&A, 417, 651

Skrutskie, M. F., Cutri, R. M., Stiening, R., et al. 2006, AJ, 131, 1163

Sneden, C. A. 1973, PhD thesis, the University of Texas at Austin

Soderblom, D. R. 2010, ARA\&A, 48, 581

Soderblom, D. R., Jones, B. F., Balachandran, S., et al. 1993, AJ, 106, 1059

Soderblom, D. R., Jones, B. F., Stauffer, J. R., \& Chaboyer, B. 1995, AJ, 110, 729

Soderblom, D. R., King, J. R., Hanson, R. B., et al. 1998, ApJ, 504, 192

Soderblom, D. R., Nelan, E., Benedict, G. F., et al. 2005, AJ, 129, 1616

Soderblom, D. R., Oey, M. S., Johnson, D. R. H., \& Stone, R. P. S. 1990, AJ, 99, 595

Spite, F., Spite, M., Peterson, R. C., \& Chaffee, F. H., Jr. 1987, A\&A, 171, L8

Stauffer, J. R., Hartmann, L. W., Fazio, G. G., et al. 2007, ApJS, 172, 663

Stauffer, J. R., Jones, B. F., Backman, D., et al. 2003, AJ, 126, 833

Stoughton, C., Lupton, R. H., Bernardi, M., et al. 2002, AJ, 123, 485

Taylor, B. J. 1980, AJ, 85, 242

Thorburn, J. A., Hobbs, L. M., Deliyannis, C. P., \& Pinsonneault, M. H. 1993, ApJ, 415, 150

Torres, G., Fischer, D. A., Sozzetti, A., et al. 2012, ApJ, 757, 161

Valenti, J. A., \& Fischer, D. A. 2005, ApJS, 159, 141 (VF05)

Valenti, J. A., \& Piskunov, N. 1996, A\&AS, 118, 595

van Leeuwen, F. 1999, A\&A, 341, L71

van Leeuwen, F. 2005, A\&A, 439, 805

van Leeuwen, F. 2007a, Hipparcos, the New Reduction of the Raw Data

(Dordrecht: Springer)

van Leeuwen, F. 2007b, A\&A, 474, 653

van Leeuwen, F. 2009, A\&A, 497, 209

van Leeuwen, F., \& Fantino, E. 2005, A\&A, 439, 791

Yanny, B., Rockosi, C., Newberg, H. J., et al. 2009, AJ, 137, 4377

York, D. G., Adelman, J., Anderson, J. E., Jr., et al. 2000, AJ, 120, 1579 